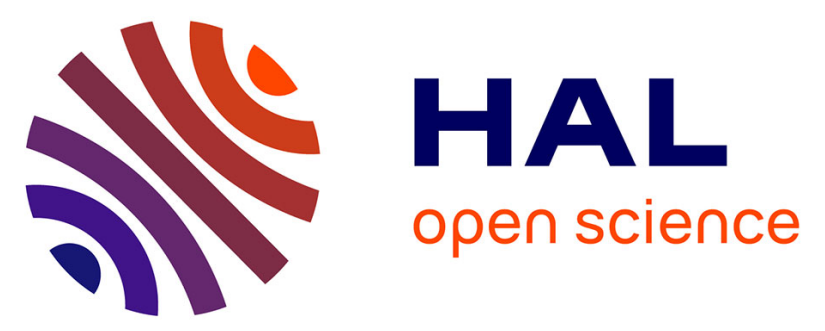

\title{
How glucosylation triggers physical-chemical properties of Curcumin: an experimental and theoretical study
}

Rois Benassi, Erika Ferrari, Sandra Lazzari, Francesca Pignedoli, Ferdinando Spagnolo, Monica Saladini

\section{- To cite this version:}

Rois Benassi, Erika Ferrari, Sandra Lazzari, Francesca Pignedoli, Ferdinando Spagnolo, et al.. How glucosylation triggers physical-chemical properties of Curcumin: an experimental and theoretical study. Journal of Physical Organic Chemistry, 2010, 24 (4), pp.299. 10.1002/poc.1750 . hal00599802

\section{HAL Id: hal-00599802 \\ https://hal.science/hal-00599802}

Submitted on 11 Jun 2011

HAL is a multi-disciplinary open access archive for the deposit and dissemination of scientific research documents, whether they are published or not. The documents may come from teaching and research institutions in France or abroad, or from public or private research centers.
L'archive ouverte pluridisciplinaire HAL, est destinée au dépôt et à la diffusion de documents scientifiques de niveau recherche, publiés ou non, émanant des établissements d'enseignement et de recherche français ou étrangers, des laboratoires publics ou privés. 


\section{Journal of Physical Organic Chemistry}

WILEY

\section{How glucosylation triggers physical-chemical properties of Curcumin: an experimental and theoretical study}

\begin{tabular}{|c|c|}
\hline Journal: & Journal of Physical Organic Chemistry \\
\hline Manuscript ID: & POC-09-0283.R2 \\
\hline Wiley - Manuscript type: & Research Article \\
\hline $\begin{array}{r}\text { Date Submitted by the } \\
\text { Author: }\end{array}$ & 29-Mar-2010 \\
\hline Complete List of Authors: & $\begin{array}{l}\text { Benassi, Rois; University of Modena and Reggio Emilia, Chemistry } \\
\text { Ferrari, Erika; University of Modena and Reggio Emilia,, Chemistry } \\
\text { Lazzari, Sandra; University of Modena and Reggio Emilia, Chemistry } \\
\text { Pignedoli, Francesca; University of Modena and Reggio Emilia, } \\
\text { Chemistry } \\
\text { Spagnolo, Ferdinando; University of Modena and Reggio Emilia, } \\
\text { Chemistry } \\
\text { Saladini, Monica; University of Modena and Reggio Emilia, } \\
\text { Chemistry }\end{array}$ \\
\hline Keywords: & Curcumin, DFT, keto-enolic structure, glucosyl-curcuminoids \\
\hline
\end{tabular}

\section{(s) ScholaroNE" \\ Manuscript Central}


1

2

3

4

5

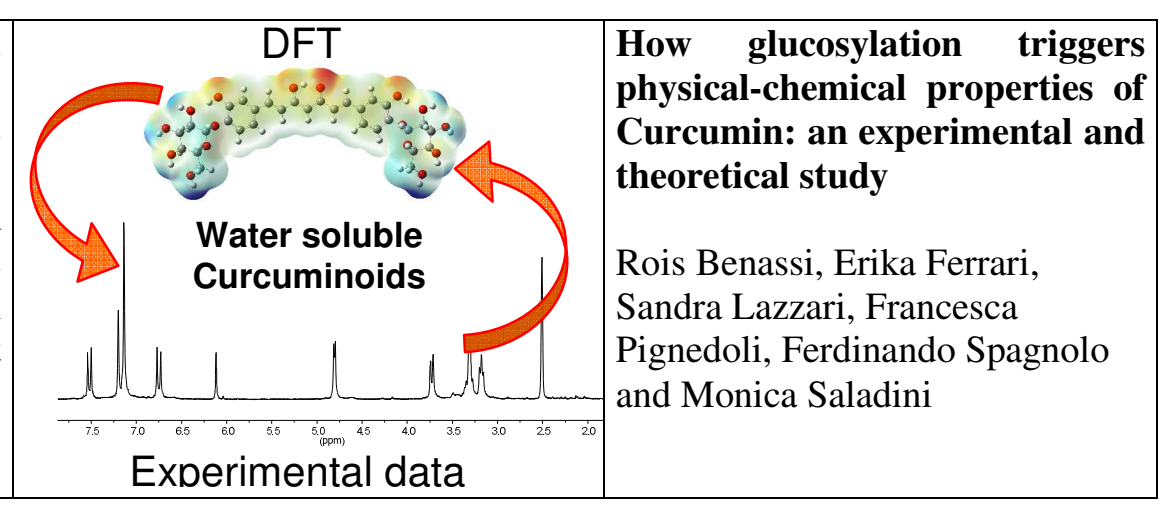




\title{
How glucosylation triggers physical-chemical properties of Curcumin: an experimental and theoretical study
}

\author{
Rois Benassi ${ }^{*}$, Erika Ferrari, Sandra Lazzari, Francesca Pignedoli, Ferdinando Spagnolo, Monica \\ Saladini \\ Department of Chemistry, University of Modena and Reggio Emilia, via Campi 183, 41100 \\ Modena, Italy
}

\begin{abstract}
In the present study we investigate the structures of glucosylated curcumin derivatives with DFT at B3LYP/6-31G* level. A conformational analysis is performed in order to determine the GS (conformational minimum) and TS (rotational transition state) of curcumin derivatives and then their electronic features are evaluated. HOMO and LUMO frontier orbitals and Maps of Electron Density Potential (MEPs) are plotted and compared. In order to correlate their predicted spectroscopic properties with IR, UV-vis and NMR experimental data we extended the theoretical study on electronic properties to different solvents $\left(\mathrm{H}_{2} \mathrm{O}, \mathrm{MeOH}, \mathrm{ACN}, \mathrm{DMSO}\right)$. The main finding is that the curcuminic core maintains the same geometrical and electronic structure in all compounds miming the metal coordination capability showed by curcumin, therefore we may confirm that the presence of glucose does not affect electronic properties of the derivatives.

Keywords: Curcumin, DFT, $\beta$-keto-enolic structure, glucosyl-curcuminoids
\end{abstract}

*Corresponding author: tel +39 0592055046, Fax +39 059373543, e-mail: rois.benassi@ unimore.it 


\section{Introduction}

Curcumin (1,7-bis(4-hydroxy-3-methoxyphenyl)-1,6-heptadiene-3,5-dione), a yellow spice extracted from Curcuma Longa L. rhizomes, is used in a wide range of applications, from industrial dyes to pharmaceutical treatments. ${ }^{[1-3]}$ It is proved that curcumin holds selective metal-chelating properties that are pharmaceutically interesting. ${ }^{[4]}$ In the field of medicinal chemistry one of the most promising properties of curcumin is its metal ligating ability towards Gallium and Iron. ${ }^{[5,6]}$ This feature can be exploited for a variety of pharmaceutical aims like metal overload detoxification, metal delivery and radio imaging. Despite these potential pharmaceutical applications, curcumin has low water solubility and limited bioavailability that makes it difficult to handle for pharmaceutical use. To improve chemical properties of curcumin several derivatives were synthesized and studied by means of theoretical and experimental data. ${ }^{[7,8]}$ The aromatic ring glucosylation was found to enhance curcumin water-solubility and kinetic stability which is a fundamental feature for drug bioavailability. ${ }^{[9]}$ The compounds were characterized and their ability to act as metal-chelating agents was also evaluated. ${ }^{[9,10]}$ Biological properties of these molecules were also tested and they showed cytotoxicity towards human ovarian carcinoma cell line leading to an improvement of Cisplatin efficacy with higher selectivity towards cancer than non-cancer cells. ${ }^{[11]}$ In order to elucidate chemical-physical properties of these molecules and to correlate their electronic structures with the ability to act as metal chelating agents, in the present study we employ DFT calculations for a conformational analysis of the compounds reported in Figure 1. A full optimization of compounds' geometry is followed by a conformational search in order to study the potential energy surface (PES) as a function of the $\mathrm{O}-\mathrm{C}$ exacyclic dihedral angle $(\phi)$ rotation (Figure1). It was reported that the presence of different solvents perturbs intra- or inter-molecular hydrogen bonds in curcumin, modifying its photophysical behavior. ${ }^{[12]}$ Therefore now we perform also a theoretical study on electronic properties of glucosyl-curcuminoids in different solvents in 
order to correlate their predicted spectroscopic properties with IR, UV-vis and NMR experimental data.

\section{Methods}

\section{$2.1 \quad$ Computational details}

The computations of all studied compounds were performed by DFT approach, the structures were fully optimized using hybrid-functional B3LYP applied to 6-31G* basis set $\left(\mathrm{B} 3 \mathrm{LYP} / 6-31 \mathrm{G}^{*}\right)^{[13-15]}$ by means of Gaussian $03^{[16]}$ package of programs.

In a previous study we described curcumin structure using both B3LYP/6-311G** and B3LYP/6-31G* levels. ${ }^{[17]}$ By comparing the obtained results, B3LYP/6-31G* showed to be a good compromise between accuracy and precision. All the calculated properties agreed well with those obtained using B3LYP/6-311G**. Therefore we decided to employ B3LYP/6-31G* in order to study the influence of glucosylation on curcumin properties. GaussView $03^{[18]}$ was used as plotting tool for data visualization.

Thermodynamics were obtained from vibrational analysis employing general procedures. The analysis of the calculated vibrational properties always confirmed the conformational minimum (GS) or rotational transition state (TS), characterized as saddle point, for the considered structure.

Atom charges were calculated from the optimized geometries at B3LYP/6-31G* level with the CHELPG approach as implemented in Gaussian03. The molecular electrostatic potential maps (MEPs) were plotted by Gaussview and reported onto 0.02 e/Bohr isosurface of electrondensity, representations of HOMO and LUMO orbital density were referred to an isovalue of 0.0004 .

The solvent effects were evaluated by employing the self-consistent reaction field (SCRF) method with polarized continuum model (PCM). ${ }^{[19-21]}$

The absorption wavelengths and oscillator strengths were calculated by means of timedependent density functional theory (TD-DFT) as implemented in Gaussian03. The magnetic 


\subsection{Conformational analysis}

Starting with the mono glucosyl-compounds, Series A (Figure1), all structures were fully optimized with the DFT approach at B3LYP/6-31G* level.

We utilized the geometries of the full reoptimized structures as the starting point for a rigid PES scan of the O-C exacyclic dihedral angle $(\phi)$ (Figure 1). The $\phi$ dihedral angle was rotated at $15^{\circ}$ step size up to a complete $360^{\circ}$ turn.

Minimum and maximum points on rigid PES were optimized and characterized as GS and TS states from the vibrational analysis. For all structures the starting point was confirmed to be the most stable structure.

Geometry of C compounds (Figure 1) was built from the more stable structure of the corresponding A series, adding a second glucose molecule. All the possible dispositions of the second sugar molecule with respect to curcumin planar skeleton were examined and optimized. Calculations on $\mathbf{C}$ compounds were then run using the same approach (rigid PES search of $\phi$ dihedral angle, optimization of the obtained structures, vibrational analysis, CHELPG charges calculation, plots of HOMO and LUMO molecular orbitals and MEPs).

\subsection{Spectroscopy}

Spectroscopic data were collected only for previously synthesized compounds $\mathbf{C}^{[10]}$

NMR spectra were recorded at $300 \mathrm{~K}$ on a Bruker Avance AMX-400 spectrometer with a Broad Band $5 \mathrm{~mm}$ probe (inverse detection). Nominal frequencies were $100.13 \mathrm{MHz}$ for ${ }^{13} \mathrm{C}$ and 400.13 MHz for ${ }^{1} \mathrm{H}$. The typical acquisition parameters for ${ }^{1} \mathrm{H}$ were as follows: $20 \mathrm{ppm}$ spectral 
bandwidth $(\mathrm{SW}), 6.1 \mu$ s pulse width $\left(90^{\circ}\right.$ pulse hard pulse on $\left.{ }^{1} \mathrm{H}\right), 0.5-1$ s pulse delay, $216-512$ number of scans. For 2D H,H-Homonuclear Correlated Spettroscopy $(\mathrm{COSY})^{[23]}$ typical parameters were used. For 2D H,X-Hetero Correlated Spettroscopy (HMBC ${ }^{[24]}$ and HMQC ${ }^{[25]}$ ) opportune parameters were used $\left(50-90^{\circ}\right.$ pulses; $32 \mathrm{k}$ data points; $1 \mathrm{~s}$ relaxation delay; $8-64 \mathrm{k}$ transients; ${ }^{1} \mathrm{~J}_{\mathrm{H}-\mathrm{C}}$ $\left.125-145 \mathrm{~Hz} ;{ }^{3} \mathrm{~J}_{\mathrm{H}-\mathrm{C}} 5-15 \mathrm{~Hz}\right)$. Methanol- $d_{4}(\mathrm{MeOD})$ and DMSO- $d_{6}$ (DMSO) were used as NMR solvent. $\mathrm{D}_{2} \mathrm{O}$ spectra showed line broadening due to hydrogen bond network forbidding their assignment.

UV-Vis measurements were performed using Jasco V-570 spectrophotometer at $25.0 \pm$ $0.1^{\circ} \mathrm{C}$ in the $200-600 \mathrm{~nm}$ spectral range employing a $1 \mathrm{~cm}$ quartz cell. $2.5 \times 10^{-5} \mathrm{M}$ solutions were prepared using different solvents: $\mathrm{H}_{2} \mathrm{O}, \mathrm{ACN}, \mathrm{DMSO}$ and $\mathrm{MeOH}$.

The infrared spectra of solid compounds were obtained by means of a Bruker FTIR VERTEX 70, with a MCT Mid-Band detector in the 4000-600 $\mathrm{cm}^{-1}$ spectral range using an ATR Golden-Gate (Heated-Diamond top-plate).

\section{Results and discussion}

\subsection{Conformational analysis}

Previous studies ${ }^{[7,17,26]}$ demonstrated that the keto-enolic tautomer of curcumin is the most stable form, so we decided to use it to describe the core of all curcumin derivatives reported in Figure 1.

The first remarkable result is that for all compounds the curcuminic core maintains a completely planar conformation with the same geometrical parameters of curcumin, ${ }^{[17]}$ allowing Curcumin-like electronic conjugation of aromatic/ $\pi$ electrons. This was found to be a fundamental feature to allow metal coordination through dissociated enolic moiety. ${ }^{[27]}$ The introduction of a glucose molecule may perturb curcumin skeleton by means of hydrogen bond interactions or conjugation effects, therefore we decided to scan the dihedral angle $\phi$ as it defines the sugar 
molecule orientation. Table 1 reports energies, thermodynamic quantities and dihedral angles $(\phi, \theta)$ values obtained from conformational analysis of all studied compounds.

A plot of $\Delta \mathrm{G}$ values as a function of $\phi$ angle for $\mathbf{A}$ type compounds is reported in Figure 2. From the analysis of rotational free energy, two states of rotational maximum $\left(\mathrm{TS}_{1}\right.$ and $\left.\mathrm{TS}_{2}\right)$ and two states of minimum $\left(\mathrm{GS}_{1}\right.$ and $\left.\mathrm{GS}_{2}\right)$ are found.

The main differences in $\phi$ values are observed for the absolute minimum conformation $\left(\mathrm{GS}_{1}\right)$, ranging from $71^{\circ}$ to $118^{\circ}$ (Table 1). The $\phi$ value increases following the decrease of aromatic substituent steric hindrance $\left(\mathrm{H}<\mathrm{OH}<\mathrm{OCH}_{3}\right) . \mathrm{GS}_{2}$ ground state is less influenced by substituent bulkiness, since the sugar moiety is rotated thus minimizing the interaction with the substituent on the aromatic ring (Figure 3). As a consequence, $\phi$ values in $\mathrm{GS}_{2}$ are similar, ranging from $282^{\circ}$ to $293^{\circ}$.

Free Activation Energy of rotation $\left(\Delta \mathrm{G}^{*}\right)$ of $\mathrm{TS}_{1}$ for $\mathbf{A 2}$ and $\mathbf{A 3}$ are similar (A2 $7.67 \mathrm{Kcal}$ $\mathrm{mol}^{-1}$; A3 $\left.7.45 \mathrm{Kcal} \mathrm{mol}^{-1}\right)$, while for $\mathbf{A 1} \Delta \mathrm{G}^{*}$ assumes an higher value $\left(9.43 \mathrm{Kcal} \mathrm{mol}^{-1}\right)$. In $\mathbf{A 1}$ the geometrical analysis of $\mathrm{GS}_{1}$ (Figure 3) provides the evidence of an hydrogen bond between $\mathrm{OH}$

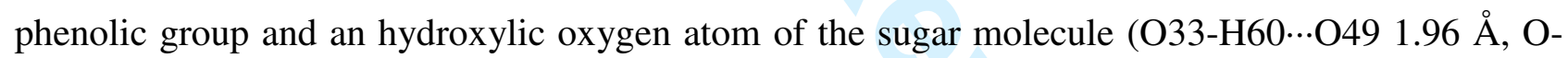
$\mathrm{H} \cdots \mathrm{O}$ angle $158.6^{\circ}$ ). The hydrogen bond breakage in $\mathbf{A 1}$ strongly contributes to the higher $\Delta \mathrm{G}^{*}$. In A2 and A3 only steric effects are responsible for the $\Delta \mathrm{G}^{*}$ value and no evidence of significant $\mathrm{H}$ bond is observed. Previous study on similar $\beta$-diketo-mono-glucosylated derivatives ${ }^{[27]}$ showed the formation of an hydrogen bond involving $\mathrm{OCH}_{3}$ aromatic substituent and glucosydic moiety ( $\mathrm{OH} \cdots \mathrm{O} 2.23 \AA$, $\mathrm{OHO}$ angle $\left.155^{\circ}\right)$. Instead in $\mathrm{A} 2$ no hydrogen interaction is observed suggesting that the greater extent of $\pi$ delocalization with respect to the previously studied compound, probably makes $\mathrm{OCH}_{3}$ group less capable to form hydrogen bond.

In di-glucosylated $\mathbf{C}$ compounds all the possible dispositions of the two sugar molecules with respect to the planar skeleton of curcumin were optimized and the results for $\mathbf{C} 2$ are reported 
in Figure 4, $\mathbf{C 1}$ and $\mathbf{C 3}$ show the same trend. The $\sin$-sin configuration is the most stable therefore it is used as the starting point for conformational analysis on $\phi$ dihedral angle. For all $\mathbf{C}$ compounds the value of $\theta$ dihedral angle results close to the corresponding $\phi$ angle in $\mathrm{GS}_{1}$ state of $\mathbf{A}$ compound with opposite sign.

C compounds show the same trend as mono-glucosylated (A series) regarding PSE. The $\Delta \mathrm{G}^{*}$ and dihedral angles for $\mathbf{A}$ and $\mathbf{C}$ homologues are superimposable with a maximum difference of $\pm 2^{\circ}$ (Table 1).

For each $\mathbf{C}$ compound, in all states of maximum and minimum, the value of the not scanned $\theta$ dihedral angle is constant and equal to the $\phi$ value obtained in the ground state $\left(\mathrm{GS}_{1}\right)$ with opposite sign. This evidence suggests that the two sugar molecules rotate independently and do not influence each other during the rotation. We suggest that $\theta$ angle can rotate with the same potential energy of $\phi$ since $\mathbf{C}$ compounds maintain the same symmetric structure observed in curcumin. For all compounds the relatively low $\Delta \mathrm{G}^{*}$ values $\left(<10.04 \mathrm{kcal} \mathrm{mol}^{-1}\right)$ allow a "free" rotation of the glucosydic group around $\phi$ and $\theta$ dihedral angle at room temperature. Anyway the free rotation of the glucose moieties does not exert any steric hindrance on keto-enolic group in any conformation, maintaining it accessible to a metal ion for the coordination reaction.

We observed also the formation of an hydrogen bond interaction between $\mathrm{OH}$ phenolic

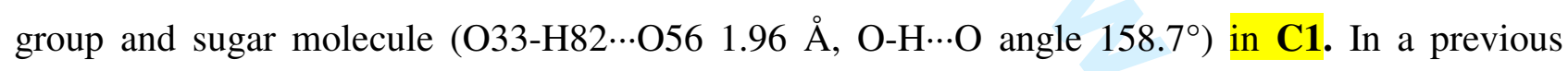
study on $\mathbf{C}$ compounds we discovered that the nature of meta substituent on the aromatic ring affects lipophilicity in the order $\mathrm{OH}>\mathrm{H}>\mathrm{OCH}_{3}{ }^{[10]}$ Today computational data may account for the unexpected increased lipophilicity of $\mathbf{C 1}$ due to the formation of the intra-molecular hydrogen bond interactions with the glucosydic moiety. 


\subsection{Molecular Orbitals}

HOMO and LUMO molecular orbitals where calculated for both GS and TS conformers for A and $\mathbf{C}$ series. Figure $\mathbf{5}$ shows HOMO and LUMO molecular orbitals at the $\mathrm{GS}_{1}$ conformation, HOMO and LUMO for the other conformations $\mathrm{GS}_{2}, \mathrm{TS}_{1}, \mathrm{TS}_{2}$ are perfectly overlapping with $\mathrm{GS}_{1}$. HOMO and LUMO are delocalized through the whole curcuminic core, allocating the electron density both on the dienic and benzenic moieties as found for curcumin. ${ }^{[17,26]}$ The introduction of one or two glucose molecules does not raise any interaction between the newly attached substituent and aromatic groups, thus not affecting the peculiar conjugation of aromatic/ $\pi$ electrons of curcumin core confirming the results of conformational analysis.

\subsection{MEPs}

Figure 6 shows the MEPs of $\mathrm{GS}_{1}$ state. In all compounds, the negative charge of the curcuminic core is located on the $\beta$-keto-enolic oxygen atoms, resembling the same trend of curcumin. ${ }^{[17,26]}$ Table 2 reports charge density values of $\beta$-keto-enolic and phenolic oxygen atoms of $\mathbf{A 1}$ and $\mathbf{C 1}$ in $\mathrm{GS}_{1}$ state. By comparing the values obtained in vacuum with those of curcumin we can observe variation of $\approx 0.004$ in the negative charge of the $\beta$-keto-enolic oxygen atoms, thus suggesting the same potential metal coordination ability of curcumin. ${ }^{[6]}$ The presence of OH groups in $\mathbf{A 1}$ and $\mathbf{C 1}$ introduces a further center of negative charge that might affects physical-chemical properties. Although enolic and phenolic oxygens show almost the same negative charge values the involvement of phenolic oxygen in metal coordination is prevented by the higher $\mathrm{pK}_{\mathrm{a}}$ value $\left[\mathrm{pK}_{\mathrm{a}}\right.$ (phenolic) 9.4; $\mathrm{pK}_{\mathrm{a}}$ (enolic) 8.2]. ${ }^{[11]}$ The negative charge on $\mathrm{OH}$ aromatic substituent evidences its strong electron withdrawing ability, which probably influences the $\mathrm{pK}_{\mathrm{a}}$ value of the $\beta$-keto-enolic group, in fact $\mathbf{C 1}$ was found to be the most acidic compound in aqueous solution and the more effective $\mathrm{Fe}(\mathrm{III})$ and $\mathrm{Ga}(\mathrm{III})$ chelating agent. ${ }^{[11]}$

A and $\mathbf{C}$ compounds show the same electronic properties (MEPs, CHELPG) suggesting similar coordination ability, therefore the analysis of solvent effects is limited to $\mathbf{C}$ compounds in 
order to compare their electronic properties with experimental data. MEPs calculated in different solvents look like those in vacuum; the corresponding density charge values are reported in Table 2.

We can observe a general increase of the negative charge on ketonic oxygen atom in solvent with respect to vacuum. This effect seems to be directly related to solvent polarity, in fact $\mathrm{H}_{2} \mathrm{O}$ gives major increase in negative charge. On the contrary the ability to form hydrogen bonds seems not to influence charge distribution. The high charge density on enolic function may account for the replacement of hydrogen atom by metal ion with a lower $\mathrm{pK}_{\mathrm{a}}$ value with respect to free ligand system, enabling metal coordination and preventing hydroxide precipitation.

MEPs do not take into account higher electrostatic effects as polarization which may be very important in metal complexation. Anyway the higher charge density on keto-enolic group with respect to sugar oxygen atoms suggests that the keto-enolic function is the only potentially involved in metal coordination. In addition chelating effect and enolic proton acidity enforces this assumption. Previous NMR study on $\mathbf{C}$ compounds ${ }^{[9]}$ established that sugar moiety was never involved in metal ligation as no ${ }^{1} \mathrm{H}$ and ${ }^{13} \mathrm{C}$ chemical shifts were observed upon metal addition.

\subsection{NMR spectroscopy}

${ }^{1} \mathrm{H}$ and ${ }^{13} \mathrm{C}$ NMR spectra were recorded for $\mathbf{C}$ compounds and were compared with the calculated values. Figure 7 reports ${ }^{1} \mathrm{H}$ NMR spectrum of $\mathbf{C 1}$ in DMSO- $d_{6}$. Experimental data evidence only one set of signals corresponding to the keto-enolic form; the complete symmetry $\left(\mathrm{C}_{2 \mathrm{v}}\right)$ of the molecule, due to resonance between the two possible keto-enolic limit structures, is supported by the equivalence of double bonds and aromatic rings, and by ${ }^{13} \mathrm{C} N \mathrm{NR}$ spectrum giving undistinguishable and equivalent peaks for ketonic and enolic carbons. $\mathbf{C 2}$ and $\mathbf{C 3}$ behave the same. The fit between experimental chemical shifts of glucosyl-curcuminoids in DMSO and MeOD for both ${ }^{1} \mathrm{H}$ (Figure 8A) and ${ }^{13} \mathrm{C}$ (Figure 9B) illustrates very small variation due to experimental conditions. 
The GIAO (Gauge-Indipendent-Atomic-Orbitals) isotropic magnetic shielding tensors $\left(\sigma_{c a l c}\right)$ were calculated for $\mathbf{C 1}, \mathbf{C 2}$ and $\mathbf{C 3}$ in vacuum, $\mathrm{H}_{2} \mathrm{O}, \mathrm{MeOH}$, and DMSO (Table 1-3, supplementary material). Bearing in mind the experimental molecular symmetry of glucosyl-curcuminoids, the average value of $\sigma_{\text {calc }}\left(\bar{\sigma}_{\text {calc }}\right)$ between the two keto-enolic limit structures was calculated and reported in Table 3 for each solvent system.

${ }^{13} \mathrm{C}$ and ${ }^{1} \mathrm{H}$ experimental chemical shift assignments were supported by $2 \mathrm{D}$ COSY, HSQC and HMBC experiments, data are reported in Table 4 and Table 5.

Plotting the experimental ${ }^{13} \mathrm{C}$ and ${ }^{1} \mathrm{H}$ chemical shifts $\left(\delta_{\exp }\right)$ in MeOD and DMSO versus the $\bar{\sigma}_{\text {calc }}$ in vacuum or in the same solvent system, a linear regression is observed: $\delta_{\exp }=\mathrm{a} \cdot \bar{\sigma}_{\text {calc }}+\mathrm{b}\left(\mathrm{a}, \mathrm{b}\right.$ and $\mathrm{R}^{2}$ parameters are given in Table 4 and Table 5.). This relationship is then used to predict the chemical shifts $\left(\delta_{\text {pred }}\right) .{ }^{[17,28]}$ As already observed, ${ }^{[29]}$ the correlation between experimental chemical shifts and calculated isotropic screening constants is better for ${ }^{13} \mathrm{C}$ than for ${ }^{1} \mathrm{H}$, in fact the correlation coefficient $\mathrm{R}^{2}$ ranges from 0.9834 to 0.9939 for ${ }^{13} \mathrm{C}$ and from 0,9186 to 0.9718 for ${ }^{1} \mathrm{H}$.

A comparison with previously reported Curcumin predicted ${ }^{13} \mathrm{C}$ NMR data ${ }^{[18]}$ highlights how for glucosyl-curcuminoids the presence of the sugar moiety experiences the solvent effect, in fact linear regressions are definitely better when calculated on the basis of $\bar{\sigma}_{\text {calc }}$ in the same solvent system than in vacuum.

${ }^{1} \mathrm{H} \delta_{\text {pred }}$ values (Table 5) have good correlation with $\delta_{\exp }$ with exception of $\mathrm{H}-1$, due to the fact that the GIAO $\sigma_{c a l c}$ has almost the same value in the two resonance limit structures of ketoenolic moiety, as a consequence electronic delocalization can't be predicted accurately. Table $\mathbf{5}$ data confirm the importance of solvent environment in predicting correct ${ }^{1} \mathrm{H} \delta_{\text {pred }}$, in fact the gap in $\mathrm{R}^{2}$ value between in vacuum and solvent prediction is higher for ${ }^{1} \mathrm{H}(0.0117-0.0319)$ than ${ }^{13} \mathrm{C}$ (0.0003-0.0044). 
$\Delta \mathrm{R}^{2}$ values $\left(\mathrm{R}_{\text {solvent }}{ }^{-} \mathrm{R}^{2}\right.$ vacuum $)$ prove that solvent has a greater effect in $\mathbf{C} \mathbf{1}$ and $\mathbf{C 2}$ than in

C3; ${ }^{1} \mathrm{H}$ NMR data of $\mathbf{C 1}$ are better predicted in $\mathrm{MeOH}$ than in DMSO, $\mathbf{C 3}$ behaves in the opposite way while for $\mathbf{C 2}$ the two solvents show the same effect. This behavior suggests that the presence of $\mathrm{OH}$ and $\mathrm{OCH}_{3}$ group in $\mathbf{C 1}$ and $\mathbf{C 2}$ respectively may interact with solvent through hydrogen bond and/or dipolar interactions.

Figure 9 reports the plotting of ${ }^{1} \mathrm{H}$ and ${ }^{13} \mathrm{C} \delta_{\text {exp }}$ vs. $\bar{\sigma}_{\text {calc }}$ for $\mathbf{C 1}$ together with the linear relationships. $\mathbf{C 1}$ behaves differently from $\mathbf{C 2}$ and $\mathbf{C 3}$ as it concerns anomeric proton (H-11), in fact this proton is not well predicted by calculation as a consequence of the intra-molecular hydrogen bond between the $\mathrm{OH}$ phenolic group and the sugar moiety.

\subsection{UV-Vis spectroscopy}

Each compound shows a strong intense absorption band in the 300-500 nm wavelength region. Absorption maxima are reported in Table 6 together with calculated values in the corresponding solvent. The red shift in DMSO with respect to ACN medium follows the order $\mathbf{C 1}>\mathbf{C 2}>\mathbf{C 3}$ and it is related to the higher polarity of the phenolic substituent $\left(\mathrm{OH}>\mathrm{OCH} \mathrm{H}_{3}>\mathrm{H}\right)$ interacting with different polar solvent. A solvent dependent red shift in maximum was found also in curcumin ${ }^{[12]}$ and was related to solvent polarity and proticity, in fact methanol, a strong hydrogen bond-donating as well as hydrogen bond accepting gave the greatest maximum red shift. In our case the three compounds in $\mathrm{H}_{2} \mathrm{O}$ and $\mathrm{MeOH}$ behaves similarly, probably because the hydrogen bond effect is masked by the presence of sugar moiety. The same trend is observed in predicted values.

\subsection{Infra Red Spectroscopy}

Usually the calculated harmonic vibrational wavenumbers are higher than the experimental ones, because of the anharmonicity, incomplete treatment of electron correlation and use of finite 
one-particle basis set. ${ }^{[29]}$ A linear relationship with a good correlation coefficient is obtained plotting the experimental wavenumbers versus the calculated ones $\left(\bar{v}_{\text {calc }}\right)$ for all $\mathbf{C}$ compounds; Figure 10 reports $\mathbf{C 1}$ data. This result suggests that the over-estimation of calculated wave numbers is quite systematic and allows to predict FT-IR spectra. The slope of the linear regression $(\mathrm{a}=$ 0.9991) is greater than the value (0.9613) estimated at B3LYP/6-31G* level for a great number of different compounds. ${ }^{[30]}$ A list of the experimental and calculated frequencies together with a tentative assignment is reported as supplementary material (Table 4 supplementary material)

\section{Conclusions}

We have demonstrated that the addition of bulky sugar molecule does not affect electronic properties of curcuminic core, allowing metal coordination through keto-enolic moiety. Glucose molecule interacts only with meta-substituent on aromatic ring influencing lipophilicity.

Solvent effect is a critical aspect in predicting spectroscopic data which well agree with experimental ones.

\section{Acknowledgments}

We are thankful to the "Consorzio Interuniversitario per il Calcolo Automatico dell'Italia Nord Orientale - CINECA" and to the "Laboratorio di Calcolo Scientifico Avanzato Interdipartimentale dell'Università degli Studi di Modena e Reggio Emilia" for computing facilities. We are grateful to the "Centro Interdipartimentale Grandi Strumenti - C.I.G.S." of the University of Modena and Reggio Emilia and to the "Fondazione Cassa di Risparmio di Modena" for supplying NMR spectrometer. 


\section{References}

1. S. Goel, B. Jhurani, B. Aggarwal, Mol. Nutr. Food Res. 2008; 52, 1010.

2. R.A. Sharma, A.J. Gescher, W.P. Steward, Eur. J. Cancer 2005; 4, 1955.

3. R.K. Maheshwari, A.K. Singh, J. Gaddipati, R.C. Srimal, Life Sci. 2006; 78, 2081.

4. $\quad$ L. Shen, H.F. Ji, Spectrochim. Acta A Mol. Biomol. Spectrosc. 2007; 67,619.

5. Y. Jiao, J. Wilkinson IV, X. Di, W. Wang, H. Hatcher, N.D. Kock, R. D’Agostino Jr, M.A. Knovich, F.M. Torti, S.V. Torti, Blood 2009; 113462.

6. M. Borsari, E. Ferrari, R. Grandi, M. Saladini, Inorg. Chim. Acta 2002; 328, 61.

7. P. Cornago, R.M. Claramount, L. Bouissane, I. Alkorta, J. Elguero, Tetrahedron 2008; 8089.

8. V. Bertolasi, V. Ferretti, P. Gilli, X. Yao, C. J. Li, New J. Cem. 2008; 32, 694.

9. B. Arezzini, M. Ferrali, E. Ferrari, R. Grandi, S. Monti, M. Saladini, Eur. J. Inorg. Chem. 2004; 3, 646.

10. E. Ferrari, B. Arezzini, M. Ferrali, S. Lazzari, F. Pignedoli, F. Spagnolo, M. Saladini, BioMetals 2009; 22, 701.

11. E. Ferrari, S. Lazzari, G. Marverti, F. Pignedoli, F. Spagnolo, M. Saladini, Biorg. Med. Chem. 2009; 17, 3043.

12. S.M. Khopde, K.I. Priyadarsini, D.K. Palit, T. Mukherjee, Photochem. Photobiol. 2000; 72 , 625.

13. B. Miehlich, A. Savin, H. Stoll, H. Preuss, Chem. Phys. Lett. 1989; 157, 200.

14. W. Lee, R. Yang, G. Parr, Phys. Rev. 1988; B 37, 785.

15. A.D. Becke, J. Chem. Phys. 1993; 98, 5648.

16. M.J. Frisch, G.W. Trucks, H.B. Schlegel, G.E. Scuseria, M.A. Robb, J.R. Cheeseman, J.A. Montgomery Jr., T. Vreven, K.N. Kudin, J.C. Burant, J.M. Millam, S.S. Iyengar, J. Tomasi, V. Barone, B. Mennucci, M. Cossi, G. Scalmani, N. Rega, G.A. Petersson, H. Nakatsuji, M. Hada, M. Ehara, K. Toyota, R. Fukuda, J. Hasegawa, M. Ishida, T. Nakajima, Y. Honda, O. Kitao, H. Nakai, 
M. Klene, X. Li, J.E. Knox, H.P. Hratchian, J.B. Cross, V. Bakken, C. Adamo, J. Jaramillo, R. Gomperts, R.E. Stratmann, O. Yazyev, A.J. Austin, R. Cammi, C. Pomelli, J.W. Ochterski, P.Y. Ayala, K. Morokuma, G.A. Voth, P. Salvador, J.J. Dannenberg, V.G. Zakrzewski, S. Dapprich, A.D. Daniels, M.C. Strain, O. Farkas, D.K. Malick, A.D. Rabuck, K. Raghavachari, J.B. Foresman, J.V. Ortiz, Q. Cui, A.G. Baboul, S. Cliord, J. Cioslowski, B.B. Stefanov, G. Liu, A. Liashenko, P. Piskorz, I. Komaromi, R.L. Martin, D.J. Fox, T. Keith, M.A. Al-Laham, C.Y. Peng, A. Nanayakkara, M. Challacombe, P.M.W. Gill, B. Johnson, W. Chen, M.W. Wong, C. Gonzalez, J.A. Pople, Gaussian 03, Revision C.02, Gaussian, Inc., Wallingford, CT, 2004.

17. R. Benassi, E. Ferrari, S. Lazzari, F. Spagnolo, M Saladini, J. Mol. Struct. 2008; 892, 168.

18. R. Dennington II, T. Keyth, J. Millam, K. Eppinnett, W.L. Hovell, R. Gilliand, Gaussview, Version 3.0, Semichem, Inc.,Shawnee Mission, KS, 2003.

19. S. Miertus, E. Scrocco, J. Tomasi, Chem. Phys. 1981; 55, 117.

20. S. Miertus, J. Tomasi, Chem. Phys. 1982; 65, 239.

21. M. Cossi, V. Barone, Cammi, J. Chem. Phys. Lett. 1996; 255, 327.

22. K. Wolinski, J.F. Hinton, P.J. Pulay, J. Am. Chem. Soc. 1990; 112,8251.

23. K. Nagayama, A. Kumar, K. Wuethrich, R.R. Ernst, J. Magn. Res. 1980; 40, 321.

24. A. Bax, M.F. Summers, J. Am. Chem. Soc. 1986; 108, 2093.

25. A. Bax, R. H.Griffey, B. L. Hawkins, J. Magn. Res. 1983; 55, 301.

26. E. Benassi, F. Spagnolo, Theor. Chem. Acc. 2009; 124, 235.

27. R. Benassi, E. Ferrari, R. Grandi, S. Lazzari, M. Saladini, J. Inorg. Biochem. 2007; 101, 203.

28. Blanco, I. Alkorta, J. Elguero, Magn.Res.Chem 2007; 45, 797.

29. F. M. Szafran, E. Bartoszak-Adamska, J. Koput, Z. Dega-Szafran, J. Mol. Struct. 2007; 140, 844.

30. J.B. Foresman, A. Frisch, "Exploring Chemistry with Electronic Structure Methods: A Guide to Using Gaussian"(2 ed.), Gaussian, Inc, Pittsburgh, PA 1996. 
Table 1- $\theta$ and $\varphi$ dihedral angles, total electronic energies (E), zero point vibrational energies $(\mathrm{E}+$ ZPE), thermodynamic quantities $(\mathrm{G}, \mathrm{H})$ and relative differences calculated at B3LYP/6-31G*.

a) Not rotated dihedral angle; b) rotated dihedral angle; c) a.u. ; d) Kcal mol ${ }^{-1}$

\begin{tabular}{|c|c|c|c|c|c|c|c|c|c|c|c|}
\hline & State & $\theta^{\mathrm{a}}$ & $\phi^{\mathrm{b}}$ & $\mathbf{E}^{\mathbf{c}}$ & $\mathbf{E}+\mathbf{Z P E}^{\mathrm{c}}$ & $\mathbf{H}^{\mathbf{c}}$ & $\mathbf{G}^{\mathbf{c}}$ & $\Delta \mathbf{E}^{\mathrm{d}}$ & $\Delta \mathbf{E}+Z \mathbf{Z P E}{ }^{\mathrm{d}}$ & $\Delta \mathbf{H}^{\mathrm{d}}$ & $\Delta \mathbf{G}^{\mathrm{d}}$ \\
\hline \multirow[t]{4}{*}{ A1 } & $\mathbf{G S}_{1}$ & - & 99.89 & -1795. & 202059 & 8855 & -1795. & 0.00 & .00 & 0.00 & 0.00 \\
\hline & $\mathbf{T S}_{1}$ & - & 3.03 & -1795.675170 & -1795.188357 & -1795.153763 & -1795.257743 & 9.03 & 8.60 & 8.22 & 9.43 \\
\hline & $\mathbf{G S}_{2}$ & - & 282.23 & -1795.685569 & -1795.198340 & -1795.162987 & -1795.269849 & 2.50 & .33 & 2.43 & 1.83 \\
\hline & $\mathbf{T S}_{2}$ & - & 358.46 & -1795.675225 & -1795.188406 & -1795.153778 & -1795.257568 & 8.99 & 8.57 & 8.21 & 9.54 \\
\hline \multirow[t]{4}{*}{ A2 } & $\mathbf{G S}_{\mathbf{1}}$ & - & 71.35 & -1874.295775 & -1873.750883 & -1873.712800 & -1873.826631 & 0.00 & 0 & 0.00 & 0.00 \\
\hline & $\mathbf{T S}_{1}$ & - & 216.94 & -1874.286710 & -1873.742105 & -1873.704899 & -1873.814405 & 5.69 & 5.51 & 4.96 & 7.67 \\
\hline & $\mathbf{G S}_{2}$ & - & 293.57 & -1874.293078 & -1873.747762 & -1873.709895 & -1873.822106 & 1.69 & 1.96 & 1.82 & 2.84 \\
\hline & $\mathbf{T S}_{2}$ & - & 347.61 & -1874.288686 & -1873.744054 & -1873.706854 & -1873.816857 & 4.45 & 4.29 & 3.73 & 6.13 \\
\hline \multirow[t]{4}{*}{$\mathbf{A 3}$} & $\mathbf{G S}_{1}$ & - & 117.93 & -1645.248964 & -1644.770062 & -1644.737057 & -1644.839474 & 0.00 & 0.00 & 0.00 & 0.00 \\
\hline & $\mathbf{T S}_{1}$ & - & 215.56 & -1645.238652 & -1644.760269 & -16 & -1644.8 & 6.47 & 6.15 & 5.67 & 7.45 \\
\hline & $\mathbf{G S}_{2}$ & - & 285.75 & -1645.247768 & -1644.768673 & -1644.735734 & -1644.838272 & 0.75 & 0.87 & 0.83 & 0.75 \\
\hline & $\mathbf{T S}_{2}$ & - & 354.04 & -1645.240588 & -1644.762127 & -1644.729889 & -1644.829315 & 5.26 & 4.98 & 4.50 & 6.37 \\
\hline \multirow[t]{4}{*}{ C1 } & $\mathbf{G S}_{\mathbf{1}}$ & 0.42 & 100.33 & -2406.423034 & -2405.761907 & -2405 & -2405 & 0.00 & 0.00 & 0.00 & 0.00 \\
\hline & $\mathbf{T S}_{1}$ & -100.76 & 203.85 & -2406.408721 & -2405.748111 & -2405.702281 & -2405.832897 & 8.98 & 8.66 & 8.23 & 9.90 \\
\hline & $\mathbf{G S}_{2}$ & -100.38 & 283.45 & -2406.419166 & -2405.757980 & -2405.711444 & -2405.843949 & 2.43 & 2.46 & 2.48 & 2.96 \\
\hline & $\mathbf{T S}_{2}$ & -101.45 & 358.72 & -2406.408670 & -2405.748086 & -2405.702199 & -2405.832666 & 9.01 & 8.67 & 8.28 & 10.04 \\
\hline \multirow[t]{4}{*}{$\mathrm{C2}$} & GS & -70.29 & 70.21 & -2485.008984 & -2484.291502 & -2484. & -2484.383139 & 0.00 & 0.00 & 0.00 & 0.00 \\
\hline & $\mathbf{T S}_{1}$ & -69.85 & 216.44 & -2484.999813 & -2484.282656 & -2484.233480 & -2484.372251 & 5.75 & 5.55 & 5.03 & 6.83 \\
\hline & $\mathbf{G S}_{\mathbf{2}}$ & -70.32 & 292.82 & -2485.005972 & -2484.288132 & -2484 & -2484.379928 & 1.89 & 2.11 & 2.01 & 2.01 \\
\hline & $\mathbf{T S}_{2}$ & -70.41 & 347.01 & -2485.001852 & -2484.284795 & -2484.235586 & -2484.374735 & 4.47 & 4.21 & 3.71 & 5.27 \\
\hline \multirow[t]{4}{*}{$\mathrm{C3}$} & $\overline{\mathbf{G S}_{1}}$ & .28 & 121.21 & -2255.973714 & -2255.321410 & -2255.276831 & -2255.406096 & 0.00 & 0.00 & 0.00 & 0.00 \\
\hline & $\mathbf{T S}_{1}$ & -121.24 & 214.87 & -2255.963342 & -2255.311860 & -2255.267906 & -2255.396296 & 6.50 & 5.99 & 5.69 & 6.15 \\
\hline & $\mathbf{G S}_{2}$ & -119.54 & 289.28 & -2255.972864 & -2255.320652 & -2255.276018 & -2255.404949 & 0.53 & 0.48 & 0.52 & 0.72 \\
\hline & $\mathbf{T S}_{2}$ & -121.64 & 353.48 & -2255.965306 & -2255.313594 & -2255.269723 & -2255.396658 & 5.27 & 4.90 & 4.53 & 5.92 \\
\hline
\end{tabular}


Table 2- CHELPG charges calculated with B3LYP/6-31G* basis-set

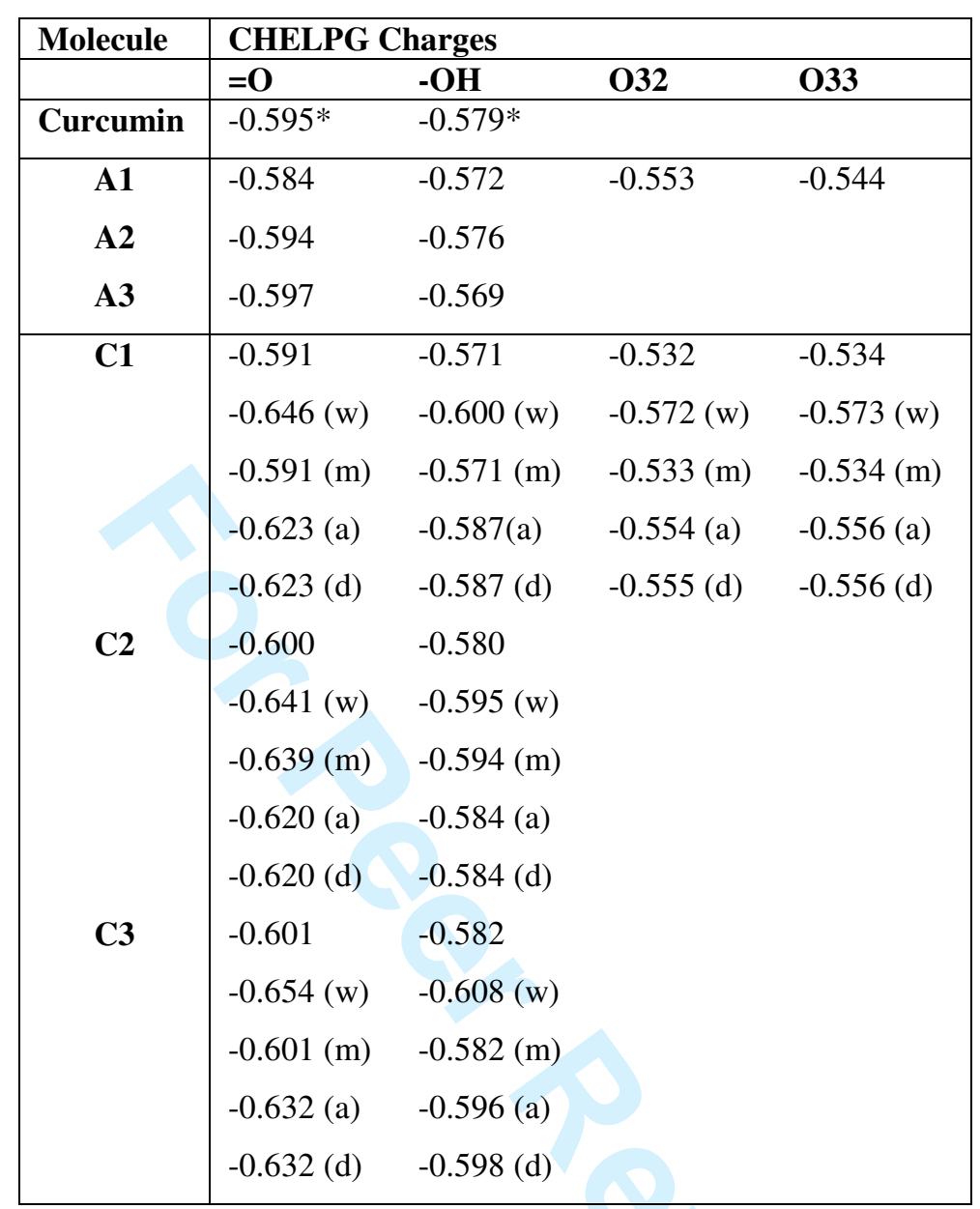

*Values taken from Ref 15. Values calculated in vacuum if not specified, (w) water, (m) MeOH, (a) ACN, (d) DMSO. 
Table 3- Calculated averaged GIAO magnetic isotropic shielding tensors $\left(\bar{\sigma}_{\text {calc }}\right)$ for $\mathbf{C 1}, \mathbf{C 2}$ and $\mathbf{C 3}$ in vacuum (v), MeOH (m) and DMSO (d).

\begin{tabular}{|c|c|c|c|c|c|c|c|c|c|}
\hline \multirow[b]{2}{*}{${ }^{13} \mathrm{C}$} & \multicolumn{3}{|c|}{ C1 } & \multicolumn{3}{|c|}{$\mathrm{C2}$} & \multicolumn{3}{|c|}{$\mathrm{C3}$} \\
\hline & $\mathrm{V}$ & $\overline{\mathrm{m}}$ & $\mathrm{d}$ & $\mathrm{V}$ & $\mathrm{m}$ & $\mathrm{d}$ & $\mathrm{v}$ & $\mathrm{m}$ & $\mathrm{d}$ \\
\hline 1 & 88.34 & 87.94 & 88.01 & 88.5 & 88.19 & 88.29 & 88.75 & 88.64 & 88.62 \\
\hline 2 & 17.99 & 17.36 & 17.65 & 18.13 & 17.46 & 17.77 & 18.14 & 17.54 & 17.82 \\
\hline 3 & 72.92 & 72.8 & 72.91 & 73.34 & 73.18 & 73.37 & 74.28 & 74.47 & 74.47 \\
\hline 4 & 54.21 & 53.84 & 54.01 & 54.69 & 54 & 54.2 & 55.27 & 54.56 & 54.82 \\
\hline 5 & 62.48 & 63.47 & 63.29 & 64.33 & 64.36 & 64.45 & 66.69 & 67.35 & 67.23 \\
\hline 6 & 73.19 & 73.98 & 73.96 & 79.16 & 77.99 & 78.37 & 60.88 & 60.51 & 60.69 \\
\hline 7 & 45.32 & 46.03 & 45.79 & 44.92 & 44.85 & 44.83 & 76.35 & 76.59 & 76.57 \\
\hline 8 & 49.42 & 48.2 & 48.58 & 48.22 & 48.5 & 48.41 & 36.48 & 35.8 & 36.03 \\
\hline 9 & 71.79 & 71.02 & 70.95 & 68.91 & 69.3 & 69.1 & 78.25 & 77.66 & 77.72 \\
\hline 10 & 81.35 & 79.7 & 80.11 & 78.51 & 77.95 & 78.11 & 70.32 & 69.18 & 69.41 \\
\hline 11 & 84.48 & 85.92 & 85.26 & 89.05 & 89.5 & 89.35 & 86.84 & 87.8 & 87.33 \\
\hline 12 & 115.66 & 116.23 & 115.72 & 115.9 & 116.54 & 115.88 & 116.26 & 116.88 & 116.26 \\
\hline 13 & 114.94 & 115.9 & 115.4 & 113.79 & 114.9 & 114.23 & 114.3 & 115.37 & 114.81 \\
\hline 14 & 119.61 & 120.01 & 119.61 & 119.83 & 120.26 & 119.81 & 120.2 & 120.63 & 120.2 \\
\hline 15 & 114.05 & 115.18 & 114.52 & 114.92 & 116.03 & 115.35 & 113.73 & 114.89 & 114.26 \\
\hline 16 & 126.04 & 126.7 & 126.47 & 126.08 & 126.87 & 126.56 & 125.87 & 126.58 & 126.31 \\
\hline${ }^{1} \mathrm{H}$ & & & & & & & & & \\
\hline 1 & 27.27 & 26.76 & 26.96 & 27.22 & 26.74 & 26.94 & 27.28 & 26.79 & 26.99 \\
\hline 3 & 26.05 & 25.64 & 25.82 & 26.02 & 25.63 & 25.82 & 26.08 & 25.7 & 25.86 \\
\hline 4 & 24.85 & 24.85 & 24.9 & 24.87 & 24.8 & 24.86 & 24.85 & 24.83 & 24.89 \\
\hline 6 & 25.57 & 25.42 & 25.57 & 25.89 & 25.52 & 25.7 & 25.1 & 24.89 & 25.01 \\
\hline 7 & I & I & I & 1 & I & 1 & 25.16 & 25.06 & 25.13 \\
\hline 9 & 25.19 & 24.91 & 25.03 & 25.16 & 25.01 & 25.12 & 25.34 & 25.06 & 25.18 \\
\hline 10 & 25.22 & 24.89 & 25.01 & 24.97 & 24.72 & 24.83 & 24.61 & 24.32 & 24.43 \\
\hline 11 & 27.82 & 26.98 & 27.53 & 26.89 & 26.73 & 26.86 & 27.36 & 26.69 & 27.15 \\
\hline 12 & 28.49 & 28.23 & 28.47 & 28.43 & 28.21 & 28.47 & 28.39 & 28.18 & 28.42 \\
\hline 13 & 28.6 & 28.19 & 28.44 & 28.7 & 28.28 & 28.57 & 28.6 & 28.17 & 28.43 \\
\hline 14 & 28.2 & 28.03 & 28.22 & 28.21 & 28.04 & 28.25 & 28.2 & 28.04 & 28.24 \\
\hline 15 & 28.94 & 28.37 & 28.69 & 29.09 & 28.53 & 28.88 & 28.87 & 28.27 & 28.6 \\
\hline 16 & 28 & 27.86 & 27.9 & 28.16 & 28 & 28.06 & 28.01 & 27.85 & 27.9 \\
\hline 16 & 28.14 & 28.12 & 28.14 & 28.41 & 28.39 & 28.42 & 28.22 & 28.2 & 28.23 \\
\hline
\end{tabular}


Table 4-Experimental $\left(\delta_{\text {exp }}\right)$ and predicted $\left(\delta_{\text {pred }}=\mathrm{a} \cdot \bar{\sigma}_{\text {calc }}+\mathrm{b}\right){ }^{13} \mathrm{C}$ chemical shifts (ppm) for $\mathbf{C 1}, \mathbf{C 2}$ and $\mathbf{C 3}$ in vacuum $(\mathrm{v}), \mathrm{MeOH}(\mathrm{m})$ and $\operatorname{DMSO}(\mathrm{d})$.

\begin{tabular}{|c|c|c|c|c|c|c|c|c|c|c|c|c|c|c|c|c|c|c|}
\hline & \multicolumn{6}{|c|}{$\mathrm{C1}$} & \multicolumn{6}{|c|}{$\mathrm{C2}$} & \multicolumn{6}{|c|}{$\mathrm{C3}$} \\
\hline & $\begin{array}{l}\delta_{\exp } \\
(\mathrm{m})\end{array}$ & $\begin{array}{r}\delta_{\text {pred }} \\
(\mathrm{v})\end{array}$ & $\begin{array}{r}\delta_{\text {pred }} \\
(\mathrm{m})\end{array}$ & $\begin{array}{r}\delta_{\exp } \\
\text { (d) }\end{array}$ & $\begin{array}{r}\delta_{\text {pred }} \\
(\mathrm{v})\end{array}$ & $\begin{array}{r}\delta_{\text {pred }} \\
(\mathrm{d})\end{array}$ & $\begin{array}{l}\delta_{\exp } \\
(\mathrm{m})\end{array}$ & $\begin{array}{r}\delta_{\text {pred }} \\
(\mathrm{v})\end{array}$ & $\begin{array}{r}\delta_{\text {pred }} \\
(\mathrm{m})\end{array}$ & $\begin{array}{r}\delta_{\exp } \\
\text { (d) }\end{array}$ & $\begin{array}{r}\delta_{\text {pred }} \\
(\mathrm{v})\end{array}$ & $\begin{array}{r}\delta_{\text {pred }} \\
(\mathrm{d})\end{array}$ & $\begin{array}{l}\delta_{\exp } \\
(\mathrm{m})\end{array}$ & $\begin{array}{r}\delta_{\text {pred }} \\
(\mathrm{v})\end{array}$ & $\begin{array}{r}\delta_{\text {pred }} \\
(\mathrm{m})\end{array}$ & $\begin{array}{r}\delta_{\exp } \\
\text { (d) }\end{array}$ & $\begin{array}{r}\delta_{\text {pred }} \\
\text { (d) }\end{array}$ & $\begin{array}{l}\delta_{\exp } \\
(\mathrm{m}) \\
\end{array}$ \\
\hline 1 & 2.80 & 01.85 & 02.50 & 1.71 & 03.19 & 03.62 & 2.80 & 02.08 & 02.56 & 1.32 & 03.50 & 103.76 & 102.8 & 102.4 & 102.8 & 01.74 & 03.82 & 04.01 \\
\hline 2 & 5.40 & .41 & 4.20 & & 0.20 & .27 & & 4.88 & & & & & 6 & 8 & 186.1 & & .87 & 1.69 \\
\hline 3 & 4.50 & 119.95 & 120.04 & 22.77 & 120.07 & 120.07 & 24.50 & 92 & 89 & 39 & 11 & 01 & 124.5 & 119.7 & 119.5 & .81 & 81 & 9.54 \\
\hline 4 & 2.10 & 41.90 & 141.98 & 0.56 & 140.55 & 140.66 & 2.10 & 1.86 & 2.12 & 69 & 140.53 & 89 & 142.1 & 142.4 & 142.6 & .31 & 0.82 & 1.10 \\
\hline 5 & .60 & .20 & .22 & & 50 & & .10 & .52 & 25 & & & & 1.6 & 128.8 & 28.0 & 78 & 20 & 7.48 \\
\hline 6 & .20 & .63 & & & & & 10 & 07 & & & & & 131.6 & 135.7 & 135.7 & .32 & .62 & 4.66 \\
\hline 7 & 7.70 & 52.34 & 53 & 7.27 & 50.28 & .61 & .60 & .36 & 152.99 & 47 & 151.23 & 10 & 118.9 & 117.2 & 117.0 & 116.94 & 7.52 & 117.24 \\
\hline 8 & 9.40 & 147.53 & 148.29 & 47.53 & 145.80 & 146.57 & 0.10 & 149.47 & 48.84 & & 147.61 & & 61.6 & 164.9 & 164.7 & 159.5 & 1.60 & 61.71 \\
\hline 9 & 118.80 & 121.27 & 122.31 & 116.31 & 121.31 & 122.20 & 17.30 & 125.13 & 124.83 & 115.42 & 124.96 & 124.67 & 118.9 & 115.0 & 115.7 & 116.94 & 115.42 & 115.97 \\
\hline 10 & 122.80 & 110.06 & 111.67 & 114.98 & 110.84 & 112.22 & 122.50 & 113.84 & 114.37 & 122.83 & 114.44 & 114.85 & 131.6 & 124.4 & 125.4 & 130.32 & 4.19 & 125.09 \\
\hline 11 & 96.30 & 106.38 & 105.70 & 101.58 & 107.42 & 106.61 & 99.40 & 101.43 & 101.32 & 99.97 & 102.90 & 102.61 & 96.5 & 104.7 & 104.4 & 100.34 & 105.93 & 105.43 \\
\hline 12 & 68.10 & 69.79 & 70.34 & 76.96 & 73.28 & 73.43 & 68.10 & 69.84 & 70.52 & 73.52 & 73.50 & 73.71 & 68.4 & 69.5 & 70.3 & 73.55 & 73.41 & 73.69 \\
\hline 13 & 71.40 & 70.64 & 70.73 & & 74.07 & 78 & 70.50 & 72.32 & 72.44 & & 75.81 & 51 & 71.2 & 71 & 72.1 & 98 & 75.57 & 75.28 \\
\hline 14 & 65.00 & 65.16 & 65.81 & 42 & 68.96 & 19 & 66.20 & 65.22 & 65.95 & 69.97 & 19 & 43 & 66.7 & 64 & 65.7 & .06 & 9.05 & 59.37 \\
\hline 15 & 70.10 & .68 & 71.74 & & 75.05 & & 69.70 & 71.00 & 71.14 & & 74.57 & 29 & 68.7 & 72.6 & 72.7 & 77.52 & 76.20 & 5.89 \\
\hline 16 & 59.20 & 57.61 & 57.86 & 60.80 & 61.92 & 61.72 & 59.20 & 57.86 & 58.12 & 61.14 & 62.35 & 62.08 & 58.3 & 58.0 & 58.5 & 61.07 & 62.78 & 62.67 \\
\hline $\bar{a}$ & & -1.174 & -1.161 & & -1.095 & -1.089 & & -1.177 & -1.161 & & -1.095 & -1.089 & & -1.195 & -1.176 & & -1.105 & -1.097 \\
\hline $\mathrm{b}$ & & 205.52 & 204.69 & & 199.90 & 199.50 & & 206.21 & 205.06 & & 200.42 & 199.93 & & 208.51 & 207.09 & & 201.92 & 201.24 \\
\hline $\mathrm{R}^{2}$ & & 0.9834 & 0.9878 & & 0.9905 & 0.9915 & & 0.9906 & 0.9917 & & 0.9879 & 0.9882 & & 0.9896 & 0.9911 & & 0.9933 & 0.9939 \\
\hline
\end{tabular}


Table 5- Experimental $\left(\delta_{\text {exp }}\right)$ and predicted $\left(\delta_{\text {pred }}=\mathrm{a} \cdot \bar{\sigma}_{\text {calc }}+\mathrm{b}\right){ }^{1} \mathrm{H}$ chemical shifts (ppm) for C1, C2 and C3 in vacuum (v). MeOH (m) and DMSO

\begin{tabular}{|c|c|c|c|c|c|c|c|c|c|c|c|c|c|c|c|c|c|c|}
\hline \multirow[b]{2}{*}{${ }^{1} \mathrm{H}$} & \multicolumn{6}{|c|}{$\mathrm{C1}$} & \multicolumn{6}{|c|}{$\mathrm{C2}$} & \multicolumn{6}{|c|}{ C3 } \\
\hline & $\begin{array}{l}\delta_{\exp } \\
(\mathrm{m})\end{array}$ & $\begin{array}{c}\delta_{\text {pred }} \\
(\mathrm{v})\end{array}$ & $\begin{array}{c}\delta_{\text {pred }} \\
(\mathrm{m})\end{array}$ & $\begin{array}{c}\delta_{\exp } \\
(\mathrm{d})\end{array}$ & $\begin{array}{c}\delta_{\text {pred }} \\
\text { (v) }\end{array}$ & $\begin{array}{c}\delta_{\text {pred }} \\
\text { (d) }\end{array}$ & $\begin{array}{l}\delta_{\exp } \\
(\mathrm{m})\end{array}$ & $\begin{array}{c}\delta_{\text {pred }} \\
\text { (v) }\end{array}$ & $\begin{array}{c}\delta_{\text {pred }} \\
(\mathrm{m})\end{array}$ & $\begin{array}{l}\delta_{\exp } \\
(\mathrm{d})\end{array}$ & $\begin{array}{c}\delta_{\text {pred }} \\
\text { (v) }\end{array}$ & $\begin{array}{c}\delta_{\text {pred }} \\
\text { (d) }\end{array}$ & $\begin{array}{l}\delta_{\exp } \\
(\mathrm{m})\end{array}$ & $\begin{array}{c}\delta_{\text {pred }} \\
\text { (v) }\end{array}$ & $\begin{array}{c}\delta_{\text {pred }} \\
(\mathrm{m})\end{array}$ & $\begin{array}{c}\delta_{\exp } \\
\text { (d) }\end{array}$ & $\begin{array}{c}\delta_{\text {pred }} \\
\text { (d) }\end{array}$ & $\begin{array}{c}\delta_{\text {pred }} \\
(\mathrm{m})\end{array}$ \\
\hline 1 & 6.01 & 5.05 & 5.27 & 6.12 & 4.96 & 5.17 & 6.02 & 5.07 & 5.31 & 6.13 & 5.03 & 5.25 & 6.01 & 4.98 & 4.97 & 6.11 & 4.89 & 5.09 \\
\hline 3 & 6.63 & 6.40 & 6.58 & 6.75 & 6.39 & 6.54 & 6.72 & 6.38 & 6.56 & 6.87 & 6.44 & 6.57 & 6.69 & 6.31 & 6.26 & 6.83 & 6.31 & 6.44 \\
\hline 4 & 7.53 & 7.74 & 7.50 & 7.52 & 7.80 & 7.64 & 7.6 & 7.63 & 7.50 & 7.6 & 7.79 & 7.71 & 7.62 & 7.67 & 7.40 & 7.61 & 7.76 & 7.60 \\
\hline 6 & 7.14 & 6.94 & 6.83 & 7.13 & 6.96 & 6.84 & 7.12 & 6.52 & 6.69 & 7.38 & 6.59 & 6.71 & 7.14 & 7.40 & 7.25 & 7.7 & 7.46 & 7.46 \\
\hline 7 & I & I & I & I & I & 1 & I & I & I & I & I & I & 7.61 & 7.33 & 7.11 & 7.09 & 7.39 & 7.32 \\
\hline 9 & 7.20 & 7.36 & 7.43 & 7.13 & 7.40 & 7.48 & 7.18 & 7.31 & 7.26 & 7.13 & 7.45 & 7.40 & 7.60 & 7.13 & 7.05 & 7.09 & 7.18 & 7.26 \\
\hline 10 & 7.07 & 7.32 & 7.45 & 7.2 & 7.37 & 7.51 & 7.18 & 7.52 & 7.59 & 7.26 & 7.67 & 7.74 & 7.14 & 7.94 & 7.92 & 7.7 & 8.04 & 8.15 \\
\hline 11 & 4.97 & 4.44 & 5.01 & 4.81 & 4.31 & 4.49 & 4.98 & 5.43 & 5.32 & 4.99 & 5.42 & 5.34 & 4.98 & 4.90 & 4.79 & 4.96 & 4.79 & 4.90 \\
\hline 12 & 3.53 & 3.69 & 3.55 & 3.30 & 3.52 & 3.37 & 3.53 & 3.75 & 3.65 & 3.27 & 3.61 & 3.44 & 3.52 & 3.76 & 3.33 & 3.27 & 3.58 & 3.38 \\
\hline 13 & 3.47 & 3.57 & 3.60 & 3.18 & 3.40 & 3.40 & 3.48 & 3.45 & 3.57 & 3.28 & 3.29 & 3.32 & 3.47 & 3.52 & 3.30 & 3.29 & 3.33 & 3.37 \\
\hline 14 & 3.44 & 4.02 & 3.79 & 3.32 & 3.87 & 3.67 & 3.43 & 3.99 & 3.84 & 3.17 & 3.87 & 3.70 & 3.42 & 3.97 & 3.55 & 3.17 & 3.80 & 3.60 \\
\hline 15 & 3.45 & 3.19 & 3.39 & 3.36 & 3.00 & 3.10 & 3.45 & 3.03 & 3.28 & 3.34 & 2.83 & 2.95 & 3.45 & 3.22 & 3.11 & 3.37 & 3.01 & 3.17 \\
\hline 16 & 3.90 & 4.24 & 3.98 & 3.72 & 4.10 & 4.05 & 3.89 & 4.04 & 3.88 & 3.67 & 3.93 & 3.92 & 3.91 & 4.18 & 3.93 & 3.69 & 4.03 & 4.00 \\
\hline $16^{\prime}$ & 3.71 & 4.08 & 3.68 & 3.48 & 3.94 & 3.76 & 3.71 & 3.77 & 3.44 & 3.45 & 3.63 & 3.50 & 3.71 & 3.94 & 3.55 & 3.46 & 3.78 & 3.61 \\
\hline $\mathrm{a}$ & & -1.110 & -1.168 & & -1.175 & -1.197 & & -1.091 & -1.131 & & -1.175 & -1.183 & & -1.107 & -1.150 & & -1.181 & -1.196 \\
\hline b & & 35.324 & 36.527 & & 37.003 & 37.442 & & 34.772 & 35.537 & & 37.019 & 37.1198 & & 35.183 & 36.005 & & 37.103 & 37.359 \\
\hline $\mathrm{R}^{2}$ & & 0.9399 & 0.9718 & & 0.9295 & 0.9553 & & 0.9232 & 0.9524 & & 0.9186 & 0.9465 & & 0.9362 & 0.9479 & & 0.9399 & 0.9611 \\
\hline
\end{tabular}


Table 6- Uv-vis band maximum position (nm) in different solvents for $\mathbf{C 1}$ compound.

\begin{tabular}{|l|c|c|c|c|c|c|c|c|c|}
\hline & Vacuum & \multicolumn{2}{|c|}{$\mathrm{H}_{2} \mathrm{O}$} & \multicolumn{2}{c|}{$\mathrm{MeOH}$} & \multicolumn{2}{c|}{ ACN } & \multicolumn{2}{c|}{ DMSO } \\
\hline & calc & exp & calc & exp & calc & exp & calc & exp & calc \\
\hline C1 & 402 & 414 & 423 & 411 & 422 & 405 & 419 & 423 & 421 \\
\hline C2 & 413 & 413 & 432 & 411 & 432 & 413 & 428 & 425 & 431 \\
\hline C3 & 403 & 408 & 425 & 406 & 425 & 405 & 420 & 416 & 423 \\
\hline
\end{tabular}




\section{Caption of Figures}

Figure 1: General structure of the studied curcumin glucosyl derivatives.

Figure 2: $\Delta \mathrm{G}$ for compounds $\mathbf{A 1}, \mathbf{A 2}$ and $\mathbf{A 3}$ as function of the rotational dihedral angle $\phi$.

Figure 3: Final optimized structures of $\mathbf{A}$ compounds at B3LYP/6-31G* level at $\mathrm{GS}_{1}$ and $\mathrm{GS}_{2}$ state. The hydrogen interaction is represented by dots.

Figure 4: Top view of $\mathbf{C 2}$ optimized structures at B3LYP/6-31G* level showing the different sugar spatial positions with respect to curcumin planar skeleton and their total energies (a.u.).

Figure 5: Representation of HOMO and LUMO orbital density for $\mathbf{A}$ and $\mathbf{C}$ series at B3LYP/6$31 \mathrm{G}^{*}$ level.

Figure 6: Molecular electrostatic maps in vacuum of $\mathbf{A}$ and $\mathbf{C}$ compounds in their $\mathrm{GS}_{1}$ state at B3LYP/6-31G* level.

Figure 7: ${ }^{1} \mathrm{H}$ NMR spectrum of $\mathbf{C 1}$ in DMSO- $d_{6}\left({ }^{*}\right.$ residual DMSO)

Figure 8: Plots of DMSO- $d_{6} v s . \mathrm{MeOD}$ experimental ${ }^{1} \mathrm{H}(\mathbf{A})$ and ${ }^{13} \mathrm{C}(\mathbf{B})$ chemical shifts $(\delta)$ for glucosyl-curcuminoids: C1 ( $\nabla)$. C2 ( $\square)$ and $\mathbf{C 3}(\circ)$.

Figure 9: Plot of ${ }^{1} \mathrm{H}$ and ${ }^{13} \mathrm{C} \delta_{\text {exp }}$ vs. $\bar{\sigma}_{\text {calc }}$ for $\mathbf{C 1}$. Solid circle is referred to $\mathrm{H}-1$ proton, dotted circle highlights anomeric proton (H-11).

Figure 10: Correlation between experimental and B3LYP/6-311G* in vacuum calculated wavenumbers for C1. Equation: $\bar{\nu}_{\text {exp }}=a \cdot \bar{v}_{\text {calc }} ; \mathrm{a}=0.9991, \mathrm{R}^{2}=0.9983$. 
Figure 1

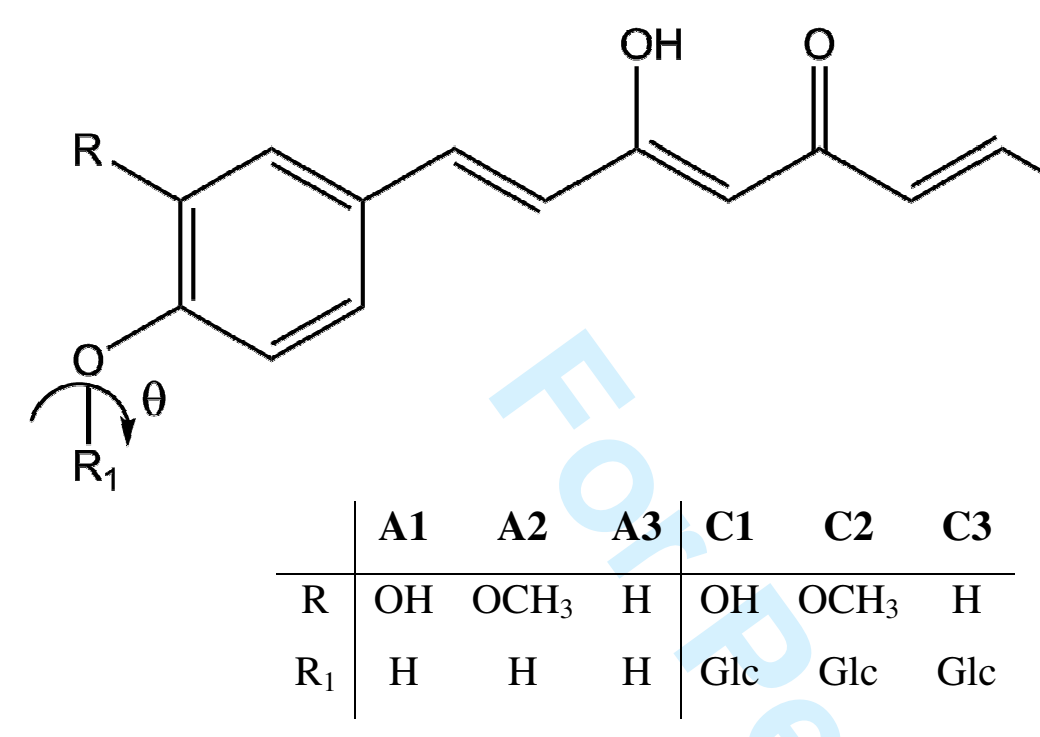<smiles>[R]c1cc(C)ccc1O</smiles>

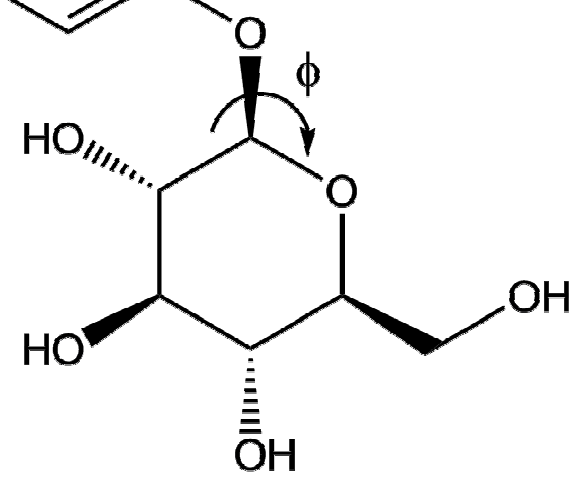


Figure 2

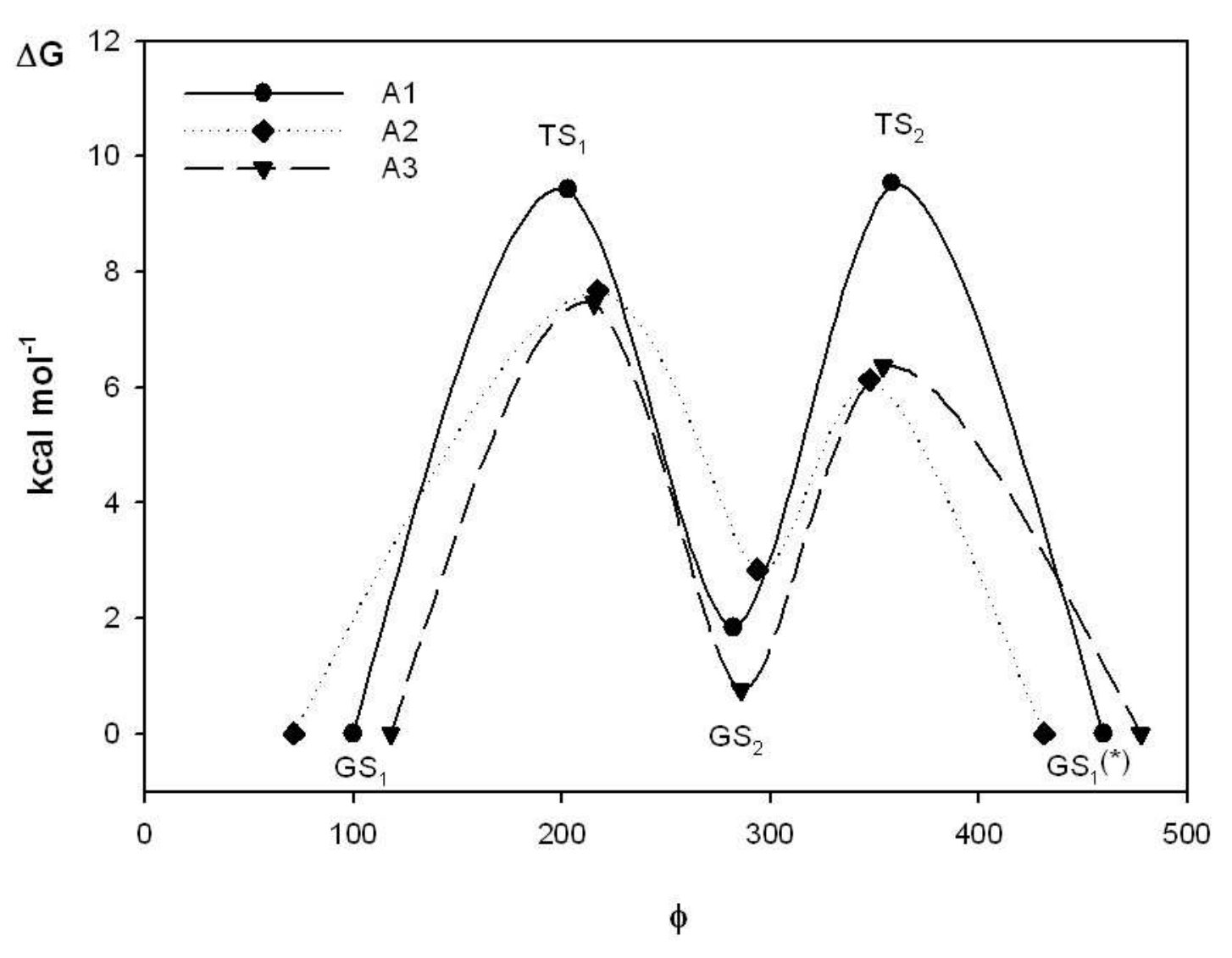




\section{Figure 3}

$$
\text { (5) }
$$$$
\text { (51) }
$$$$
\text { A2 in } \mathrm{GS}_{1}
$$$$
\mathrm{A}^{2} \text { in } \mathrm{GS}_{2}
$$
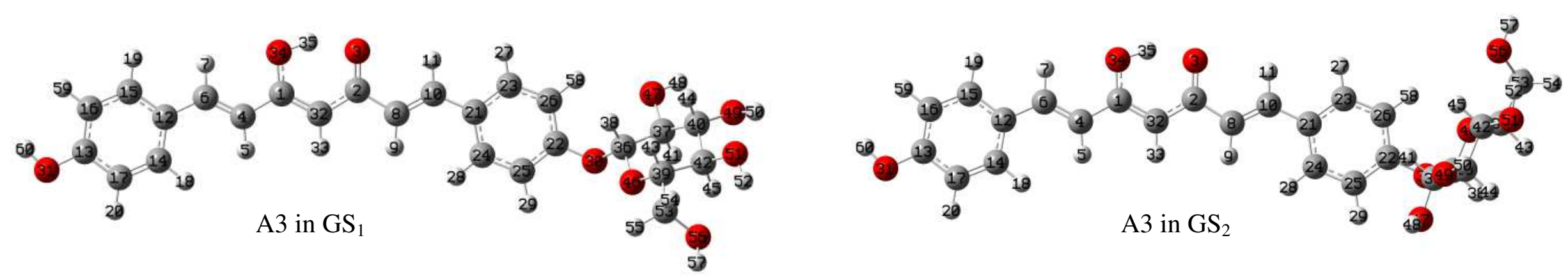
Figure 4

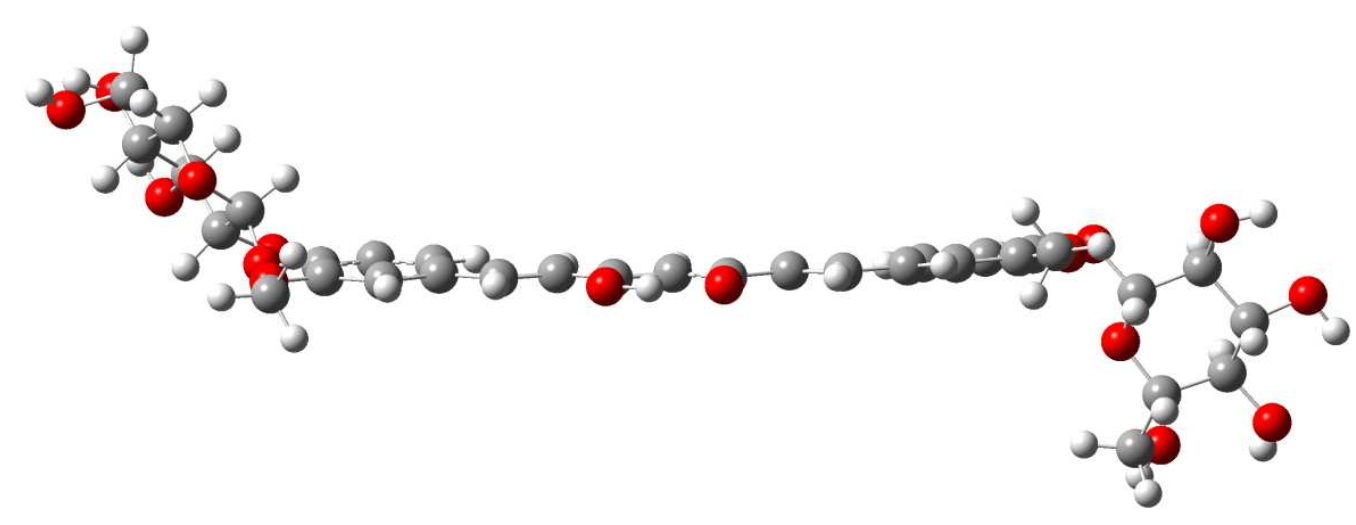

sin-anti configuration $\mathrm{E}=-2485.008088$ a.u.

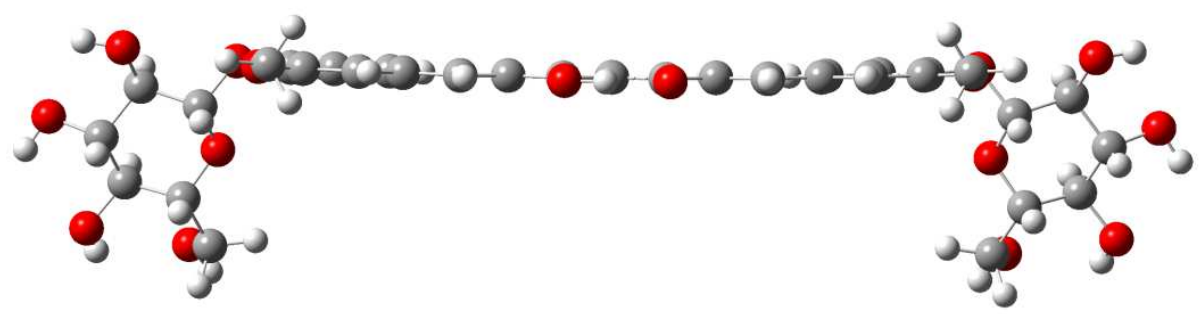

$\sin$ - $\sin$ configuraton $\mathrm{E}=-2485.008984$ a.u. 
Figure 5

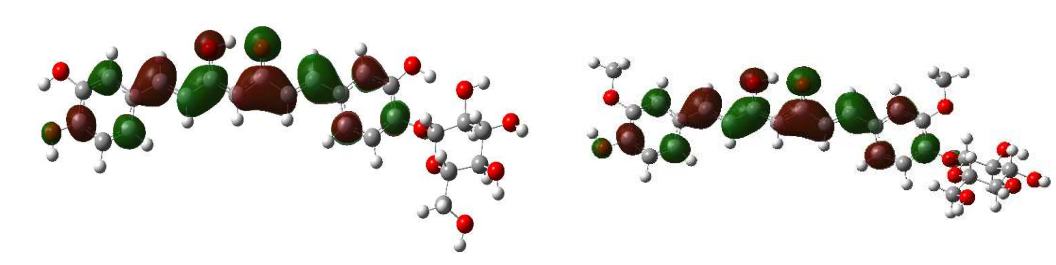
A1 HOMO
A2 HOMO
A3 HOMO

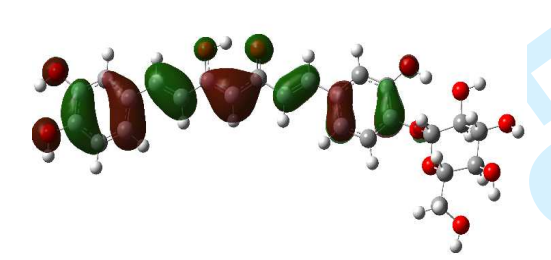

A1 LUMO
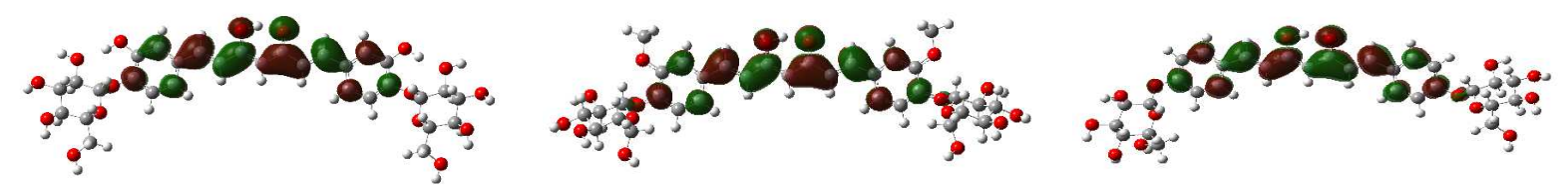

C1 HOMO

C3 HOMO

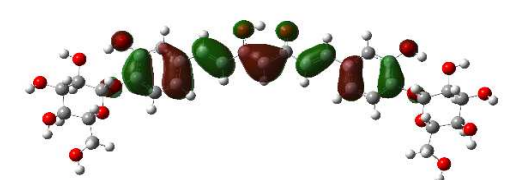

C1 LUMO
A2 LUMO

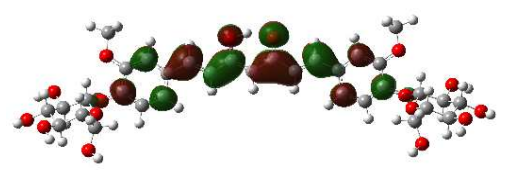

C2 HOMO
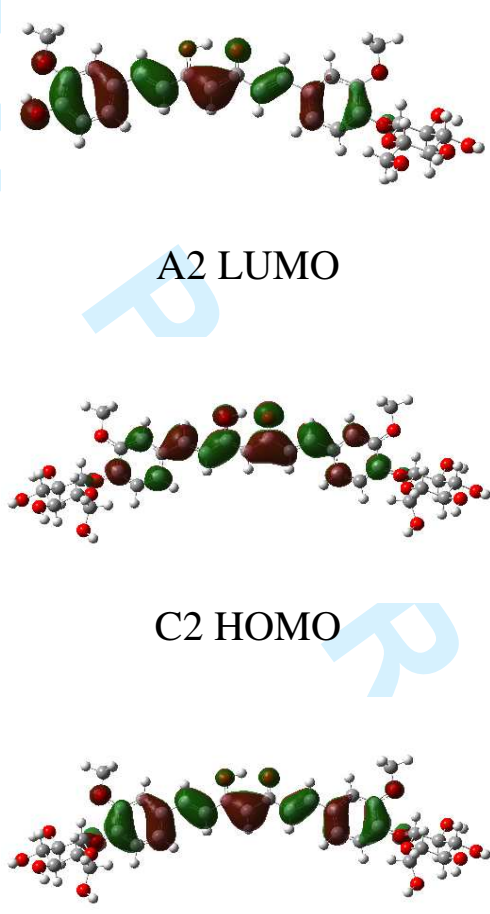

C2 LUMO
A3 LUMO

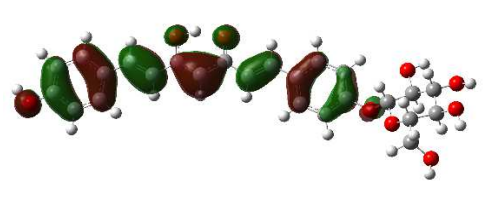


Figure 6

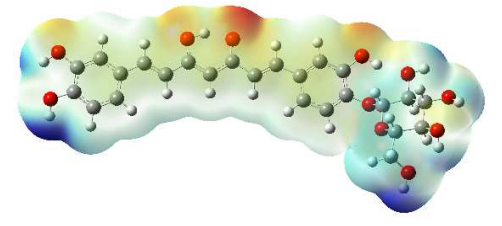

A1

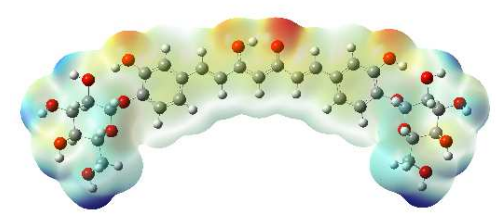

C1

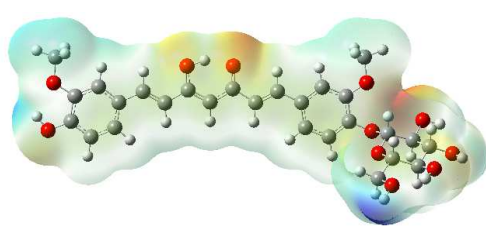

A2

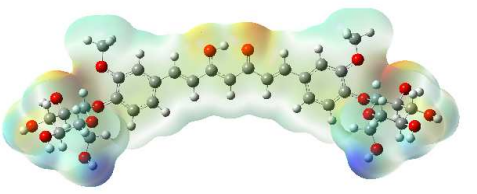

$\mathrm{C} 2$

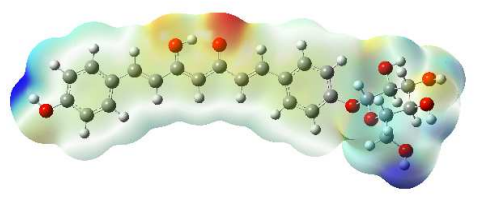

A3

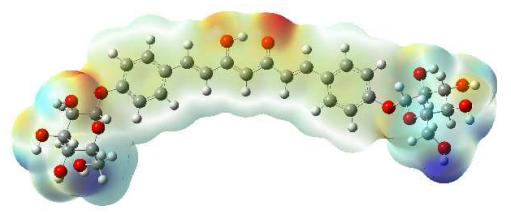

C3 
Figure 7

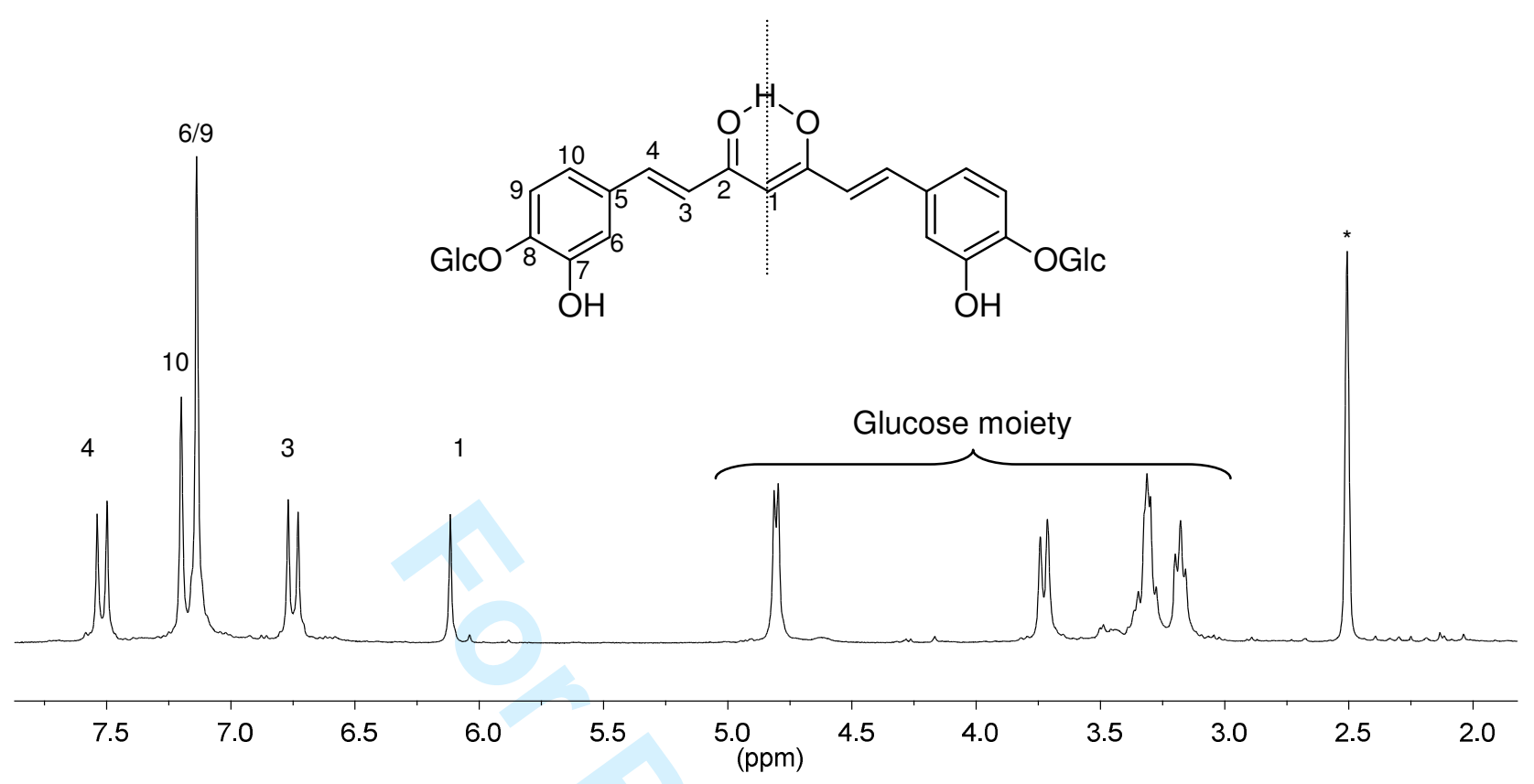




\section{Figure 8}
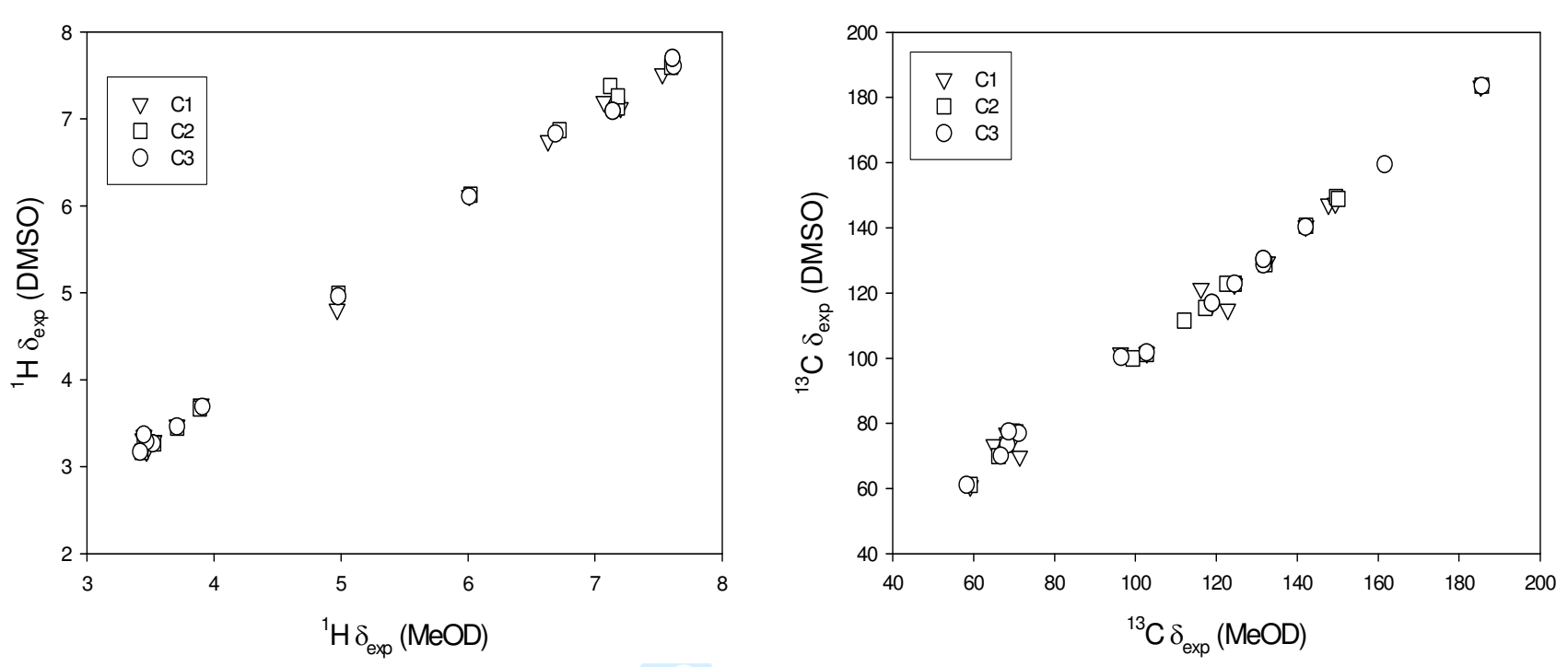

28 


\section{Figure 9}
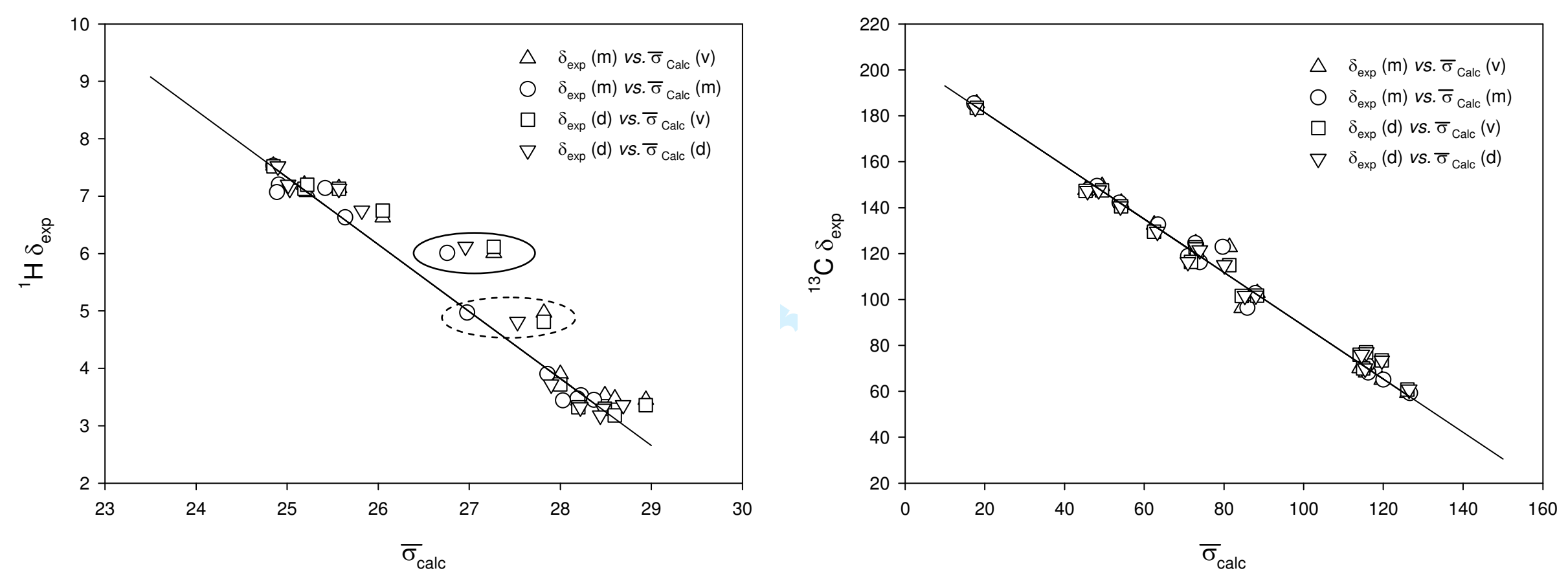
Figure 10

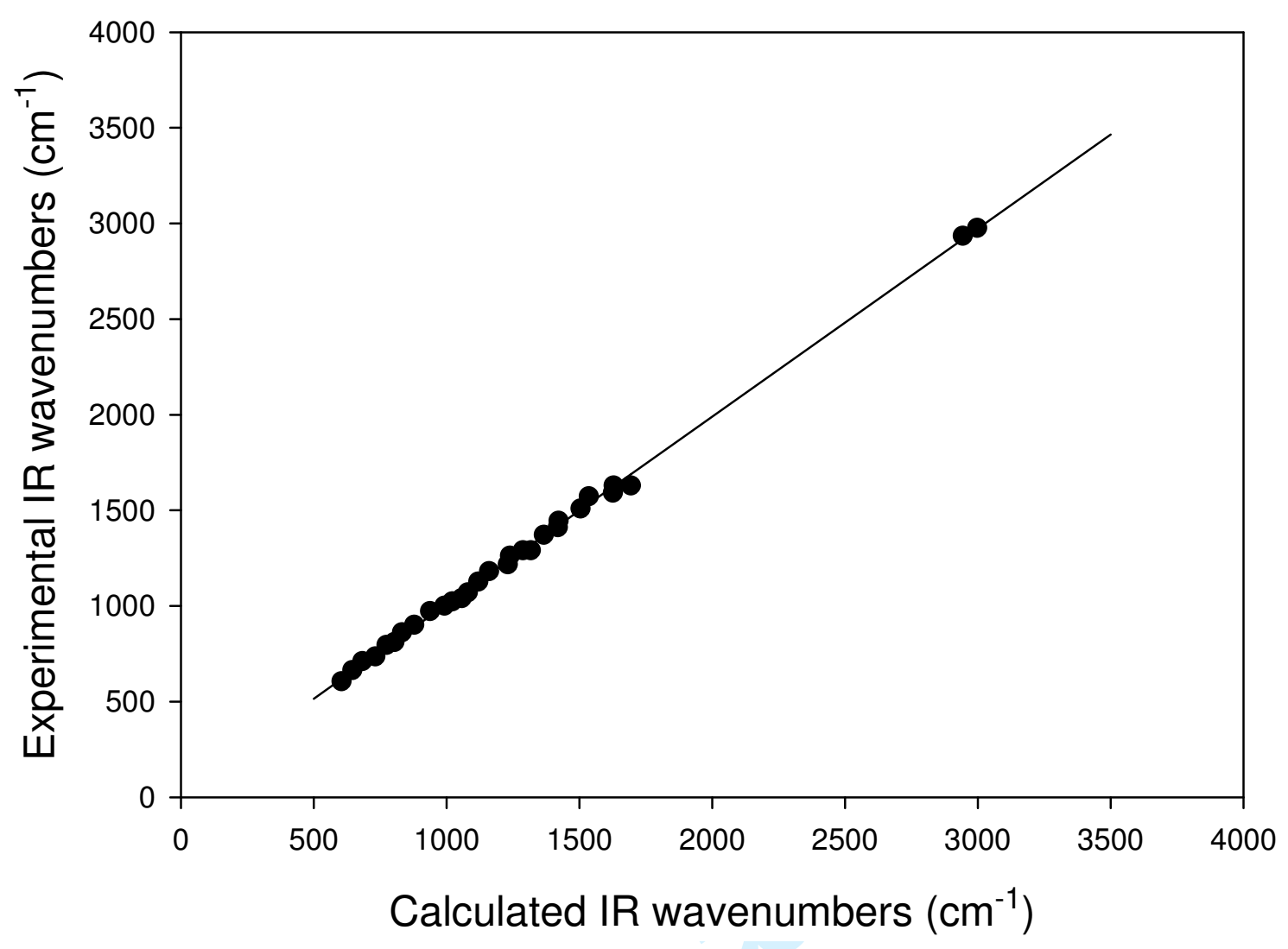


Table 1: NMR values for $\mathrm{C} 1$ compound calculated in vacuum, $\mathrm{H}_{2} \mathrm{O}, \mathrm{MeOH}, \mathrm{ACN}$ and DMSO.

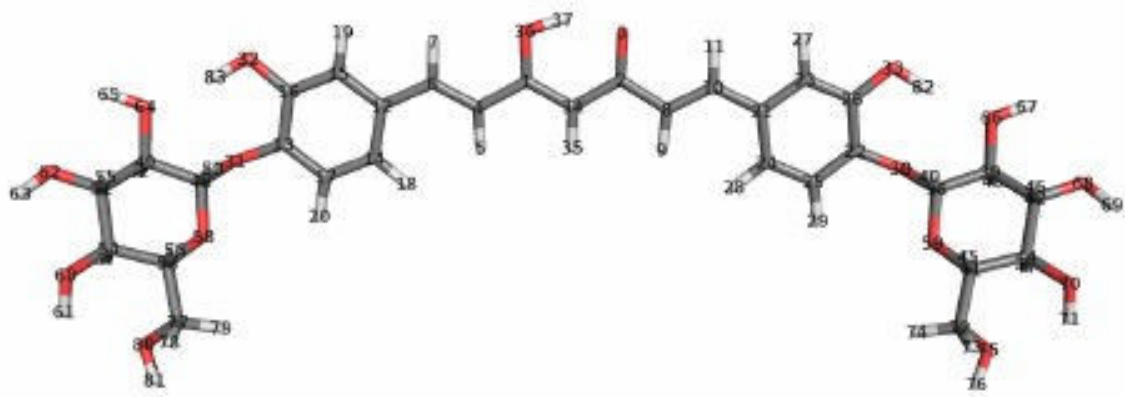

\begin{tabular}{|c|c|c|c|c|c|}
\hline ATOMN & vacuum & $\underline{\mathrm{H}_{2} \mathrm{O}}$ & $\mathrm{MeOH}$ & $\mathrm{ACN}$ & DMSO \\
\hline 1 & 25.37 & 24.94 & 24.95 & 25.17 & 25.17 \\
\hline 2 & 10.62 & 9.72 & 9.77 & 10.15 & 10.15 \\
\hline 4 & 74.48 & 74.58 & 74.63 & 74.69 & 74.69 \\
\hline 5 & 26.18 & 25.74 & 25.77 & 25.95 & 25.95 \\
\hline 6 & 55.32 & 54.75 & 54.74 & 54.92 & 54.93 \\
\hline 7 & 24.95 & 24.92 & 24.92 & 24.98 & 24.98 \\
\hline 8 & 71.37 & 70.91 & 70.98 & 71.14 & 71.14 \\
\hline 9 & 25.93 & 25.49 & 25.52 & 25.7 & 25.7 \\
\hline 10 & 53.11 & 52.98 & 52.95 & 53.11 & 53.11 \\
\hline 11 & 24.75 & 24.79 & 24.79 & 24.84 & 24.84 \\
\hline 12 & 62.48 & 63.4 & 63.38 & 63.23 & 63.23 \\
\hline 13 & 49.51 & 48.29 & 48.32 & 48.69 & 48.68 \\
\hline 14 & 81.55 & 79.78 & 79.87 & 80.3 & 80.28 \\
\hline 15 & 73.57 & 74.35 & 74.3 & 74.26 & 74.27 \\
\hline 16 & 45.3 & 46.06 & 46.03 & 45.78 & 45.78 \\
\hline 17 & 71.73 & 71.01 & 70.99 & 70.94 & 70.92 \\
\hline 18 & 25.26 & 24.9 & 24.92 & 25.05 & 25.05 \\
\hline 19 & 25.62 & 25.45 & 25.46 & 25.61 & 25.61 \\
\hline 20 & 25.19 & 24.92 & 24.92 & 25.04 & 25.03 \\
\hline 21 & 62.49 & 63.59 & 63.56 & 63.34 & 63.35 \\
\hline 22 & 49.34 & 48.06 & 48.09 & 48.49 & 48.49 \\
\hline 23 & 72.82 & 73.72 & 73.67 & 73.64 & 73.65 \\
\hline 24 & 81.16 & 79.45 & 79.54 & 79.95 & 79.94 \\
\hline 25 & 71.86 & 71.06 & 71.05 & 71 & 70.99 \\
\hline 26 & 45.36 & 46.07 & 46.04 & 45.8 & 45.81 \\
\hline 27 & 25.53 & 25.38 & 25.39 & 25.54 & 25.54 \\
\hline 28 & 25.19 & 24.84 & 24.86 & 24.99 & 24.99 \\
\hline 29 & 25.19 & 24.91 & 24.92 & 25.03 & 25.03 \\
\hline 34 & 88.34 & 87.9 & 87.95 & 88.02 & 88.02 \\
\hline 35 & 27.27 & 26.73 & 26.76 & 26.97 & 26.97 \\
\hline 36 & 189.29 & 193.45 & 193.26 & 192.29 & 192.32 \\
\hline 37 & 18.36 & 18.58 & 18.57 & 18.54 & 18.54 \\
\hline 38 & 84.49 & 86.03 & 85.93 & 85.26 & 85.27 \\
\hline 39 & 115.67 & 116.25 & 116.24 & 115.72 & 115.72 \\
\hline 40 & 27.82 & 26.92 & 26.98 & 27.54 & 27.53 \\
\hline 41 & 114.06 & 115.26 & 115.18 & 114.51 & 114.52 \\
\hline 42 & 114.93 & 115.95 & 115.9 & 115.38 & 115.39 \\
\hline 43 & 28.5 & 28.23 & 28.23 & 28.47 & 28.47 \\
\hline 44 & 119.61 & 120.01 & 120.01 & 119.62 & 119.62 \\
\hline 45 & 28.94 & 28.34 & 28.38 & 28.7 & 28.69 \\
\hline 46 & 28.6 & 28.17 & 28.19 & 28.45 & 28.45 \\
\hline 47 & 28.2 & 28.04 & 28.04 & 28.23 & 28.23 \\
\hline 48 & 84.47 & 86.02 & 85.92 & 85.26 & 85.26 \\
\hline 49 & 115.67 & 116.25 & 116.24 & 115.72 & 115.72 \\
\hline 50 & 27.83 & 26.92 & 26.99 & 27.54 & 27.54 \\
\hline
\end{tabular}


Table 1 - continued from previous page

\begin{tabular}{|c|c|c|c|c|c|}
\hline ATOMN & vacuum & $\mathrm{H}_{2} \mathrm{O}$ & $\mathrm{MeOH}$ & $\mathrm{ACN}$ & DMSO \\
\hline 51 & 114.95 & 115.96 & 115.91 & 115.39 & 115.41 \\
\hline 52 & 28.5 & 28.24 & 28.24 & 28.47 & 28.48 \\
\hline 53 & 114.05 & 115.25 & 115.18 & 114.51 & 114.52 \\
\hline 54 & 119.63 & 120.02 & 120.02 & 119.63 & 119.62 \\
\hline 55 & 28.6 & 28.17 & 28.2 & 28.45 & 28.45 \\
\hline 56 & 28.95 & 28.35 & 28.38 & 28.7 & 28.7 \\
\hline 57 & 28.21 & 28.04 & 28.04 & 28.23 & 28.23 \\
\hline 61 & 30.54 & 29.9 & 29.91 & 30.21 & 30.21 \\
\hline 63 & 30.08 & 29.55 & 29.56 & 29.87 & 29.87 \\
\hline 65 & 29.99 & 29.25 & 29.29 & 29.68 & 29.68 \\
\hline 67 & 29.99 & 29.25 & 29.29 & 29.67 & 29.67 \\
\hline 69 & 30.08 & 29.55 & 29.56 & 29.87 & 29.87 \\
\hline 71 & 30.54 & 29.9 & 29.91 & 30.21 & 30.21 \\
\hline 72 & 126.03 & 126.73 & 126.7 & 126.47 & 126.47 \\
\hline 73 & 28 & 27.85 & 27.86 & 27.91 & 27.91 \\
\hline 74 & 28.15 & 28.13 & 28.13 & 28.15 & 28.15 \\
\hline 76 & 32.03 & 30.77 & 30.81 & 31.33 & 31.32 \\
\hline 77 & 126.05 & 126.74 & 126.71 & 126.48 & 126.48 \\
\hline 78 & 28.01 & 27.86 & 27.86 & 27.91 & 27.91 \\
\hline 79 & 28.15 & 28.13 & 28.13 & 28.15 & 28.15 \\
\hline 81 & 32.03 & 30.78 & 30.82 & 31.33 & 31.32 \\
\hline 82 & 25.17 & 25.02 & 25.03 & 25.07 & 25.07 \\
\hline 83 & 25.16 & 25.02 & 25.03 & 25.07 & 25.07 \\
\hline
\end{tabular}

28 
Table 2: NMR values for $\mathrm{C} 2$ compound calculated in vacuum, $\mathrm{H}_{2} \mathrm{O}, \mathrm{MeOH}, \mathrm{ACN}$ and DMSO. Oxygen atom chemical shifts are omitted.

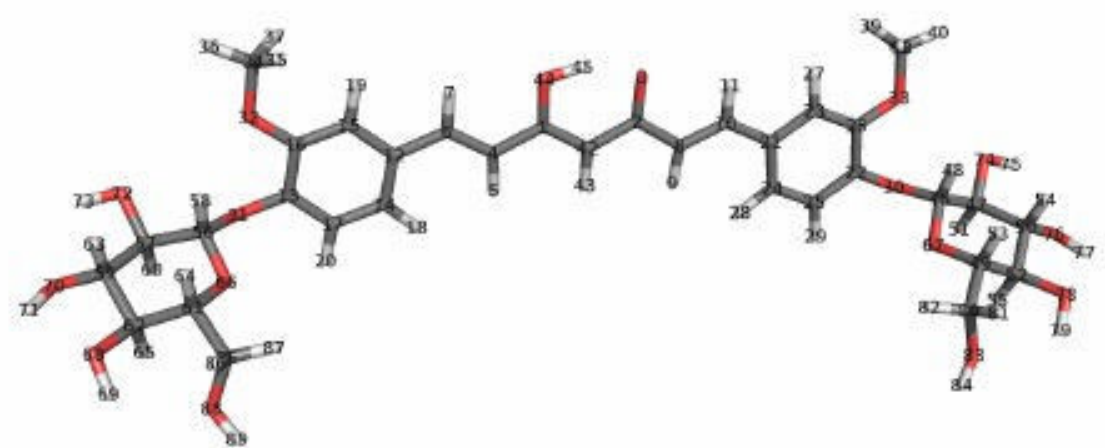

\begin{tabular}{|c|c|c|c|c|c|}
\hline ATOMN & vacuum & $\mathrm{H}_{2} \mathrm{O}$ & $\mathrm{MeOH}$ & $\mathrm{ACN}$ & DMSO \\
\hline 1 & 25.5 & 24.99 & 25.01 & 25.24 & 25.24 \\
\hline 2 & 10.76 & 9.88 & 9.93 & 10.32 & 10.32 \\
\hline 4 & 74.94 & 74.98 & 75.02 & 75.17 & 75.17 \\
\hline 5 & 26.15 & 25.74 & 25.76 & 25.95 & 25.95 \\
\hline 6 & 55.83 & 54.9 & 54.91 & 55.13 & 55.13 \\
\hline 7 & 24.98 & 24.86 & 24.87 & 24.94 & 24.94 \\
\hline 8 & 71.75 & 71.28 & 71.34 & 71.57 & 71.58 \\
\hline 9 & 25.9 & 25.49 & 25.51 & 25.69 & 25.69 \\
\hline 10 & 53.57 & 53.11 & 53.09 & 53.3 & 53.28 \\
\hline 11 & 24.77 & 24.73 & 24.73 & 24.79 & 24.78 \\
\hline 12 & 64.32 & 64.26 & 64.26 & 64.37 & 64.38 \\
\hline 13 & 48.31 & 48.6 & 48.63 & 48.52 & 48.52 \\
\hline 14 & 78.72 & 78.04 & 78.12 & 78.28 & 78.28 \\
\hline 15 & 79.53 & 78.2 & 78.3 & 78.68 & 78.68 \\
\hline 16 & 44.9 & 44.85 & 44.86 & 44.84 & 44.83 \\
\hline 17 & 68.85 & 69.41 & 69.26 & 69.07 & 69.06 \\
\hline 18 & 25.01 & 24.73 & 24.75 & 24.87 & 24.87 \\
\hline 19 & 25.95 & 25.53 & 25.56 & 25.74 & 25.74 \\
\hline 20 & 25.16 & 25.02 & 25.02 & 25.13 & 25.13 \\
\hline 21 & 64.34 & 64.46 & 64.46 & 64.51 & 64.52 \\
\hline 22 & 48.14 & 48.37 & 48.39 & 48.33 & 48.32 \\
\hline 23 & 78.8 & 77.59 & 77.68 & 78.08 & 78.07 \\
\hline 24 & 78.32 & 77.7 & 77.79 & 77.94 & 77.94 \\
\hline 25 & 68.99 & 69.48 & 69.34 & 69.15 & 69.14 \\
\hline 26 & 44.95 & 44.86 & 44.86 & 44.86 & 44.85 \\
\hline 27 & 25.85 & 25.45 & 25.48 & 25.67 & 25.67 \\
\hline 28 & 24.94 & 24.68 & 24.7 & 24.81 & 24.81 \\
\hline 29 & 25.17 & 25.01 & 25.01 & 25.13 & 25.13 \\
\hline 34 & 137.41 & 137.57 & 137.56 & 137.34 & 137.34 \\
\hline 35 & 28.57 & 28.35 & 28.36 & 28.43 & 28.43 \\
\hline 36 & 28.14 & 28.06 & 28.06 & 28.1 & 28.1 \\
\hline 37 & 28.65 & 28.51 & 28.52 & 28.59 & 28.59 \\
\hline 38 & 137.39 & 137.55 & 137.53 & 137.32 & 137.32 \\
\hline 39 & 28.63 & 28.5 & 28.5 & 28.57 & 28.57 \\
\hline 40 & 28.14 & 28.05 & 28.05 & 28.09 & 28.09 \\
\hline 41 & 28.55 & 28.33 & 28.35 & 28.42 & 28.42 \\
\hline 42 & 88.51 & 88.15 & 88.2 & 88.3 & 88.3 \\
\hline 43 & 27.23 & 26.71 & 26.74 & 26.95 & 26.95 \\
\hline 45 & 18.33 & 18.52 & 18.51 & 18.49 & 18.49 \\
\hline 46 & 89.08 & 89.77 & 89.52 & 89.37 & 89.37 \\
\hline 47 & 115.9 & 116.53 & 116.54 & 115.88 & 115.88 \\
\hline 48 & 26.89 & 26.47 & 26.72 & 26.85 & 26.86 \\
\hline 49 & 114.93 & 116.16 & 116.04 & 115.36 & 115.36 \\
\hline 50 & 113.79 & 114.93 & 114.89 & 114.23 & 114.23 \\
\hline
\end{tabular}


Table 2 - continued from previous page

1

2

\begin{tabular}{|c|c|c|c|c|c|}
\hline ATOMN & vacuum & $\mathrm{H}_{2} \mathrm{O}$ & $\mathrm{MeOH}$ & $\mathrm{ACN}$ & DMSO \\
\hline$\overline{51}$ & 28.43 & 28.23 & 28.21 & 28.47 & 28.48 \\
\hline 52 & 119.82 & 120.21 & 120.26 & 119.82 & 119.81 \\
\hline 53 & 29.09 & 28.5 & 28.54 & 28.89 & 28.89 \\
\hline 54 & 28.7 & 28.26 & 28.28 & 28.57 & 28.57 \\
\hline 55 & 28.22 & 28.07 & 28.04 & 28.24 & 28.25 \\
\hline 56 & 89.04 & 89.75 & 89.49 & 89.35 & 89.34 \\
\hline 57 & 115.91 & 116.54 & 116.56 & 115.89 & 115.89 \\
\hline 58 & 26.91 & 26.48 & 26.74 & 26.87 & 26.87 \\
\hline 59 & 113.8 & 114.94 & 114.91 & 114.24 & 114.24 \\
\hline 60 & 28.43 & 28.23 & 28.21 & 28.48 & 28.48 \\
\hline 61 & 114.92 & 116.15 & 116.04 & 115.35 & 115.35 \\
\hline 62 & 119.84 & 120.23 & 120.28 & 119.84 & 119.82 \\
\hline 63 & 28.7 & 28.27 & 28.28 & 28.58 & 28.58 \\
\hline 64 & 29.09 & 28.5 & 28.54 & 28.89 & 28.89 \\
\hline 65 & 28.22 & 28.07 & 28.04 & 28.24 & 28.25 \\
\hline 69 & 30.67 & 30.02 & 30.02 & 30.31 & 30.31 \\
\hline 71 & 30.18 & 29.59 & 29.63 & 29.93 & 29.93 \\
\hline 73 & 30.5 & 29.88 & 29.96 & 30.29 & 30.29 \\
\hline 75 & 30.5 & 29.88 & 29.95 & 30.28 & 30.29 \\
\hline 77 & 30.18 & 29.59 & 29.63 & 29.93 & 29.93 \\
\hline 79 & 30.67 & 30.01 & 30.02 & 30.31 & 30.3 \\
\hline 80 & 126.08 & 126.89 & 126.88 & 126.57 & 126.57 \\
\hline 81 & 28.17 & 28 & 28 & 28.06 & 28.06 \\
\hline 82 & 28.41 & 28.4 & 28.4 & 28.42 & 28.42 \\
\hline 84 & 32.17 & 30.85 & 30.89 & 31.42 & 31.41 \\
\hline 85 & 126.09 & 126.9 & 126.88 & 126.57 & 126.57 \\
\hline 86 & 28.17 & 28 & 28.01 & 28.06 & 28.06 \\
\hline 87 & 28.42 & 28.4 & 28.4 & 28.42 & 28.42 \\
\hline 89 & 32.17 & 30.85 & 30.89 & 31.42 & 31.41 \\
\hline
\end{tabular}


Table 3: NMR values for $\mathrm{C} 3$ compound calculated in vacuum, $\mathrm{H}_{2} \mathrm{O}, \mathrm{MeOH}, \mathrm{ACN}$ and DMSO. Oxygen atom chemical shifts are omitted.

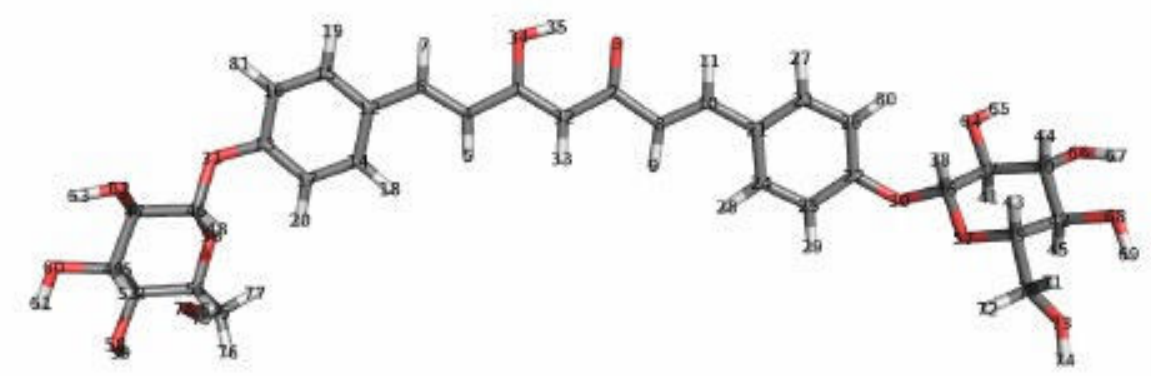

\begin{tabular}{|c|c|c|c|c|c|}
\hline ATOMN & Vacuum & $\mathrm{H}_{2} \mathrm{O}$ & $\mathrm{MeOH}$ & $\mathrm{ACN}$ & DMSO \\
\hline 1 & 25.43 & 24.95 & 24.97 & 25.18 & 25.18 \\
\hline 2 & 10.86 & 10.08 & 10.13 & 10.47 & 10.47 \\
\hline 4 & 76.13 & 76.66 & 76.7 & 76.58 & 76.58 \\
\hline 5 & 26.24 & 25.82 & 25.85 & 26.02 & 26.01 \\
\hline 6 & 56.42 & 55.38 & 55.38 & 55.71 & 55.71 \\
\hline 7 & 24.97 & 24.91 & 24.91 & 24.98 & 24.98 \\
\hline 8 & 72.44 & 72.2 & 72.25 & 72.36 & 72.36 \\
\hline 9 & 25.94 & 25.52 & 25.55 & 25.72 & 25.72 \\
\hline 10 & 54.12 & 53.76 & 53.74 & 53.96 & 53.95 \\
\hline 11 & 24.74 & 24.76 & 24.76 & 24.8 & 24.8 \\
\hline 12 & 67.23 & 67.9 & 67.89 & 67.74 & 67.74 \\
\hline 13 & 37.49 & 36.71 & 36.72 & 37.03 & 37.02 \\
\hline 14 & 70.18 & 68.76 & 68.85 & 69.07 & 69.05 \\
\hline 15 & 61.48 & 61.18 & 61.15 & 61.35 & 61.35 \\
\hline 16 & 77.04 & 77.78 & 77.69 & 77.6 & 77.61 \\
\hline 17 & 81.5 & 80.29 & 80.39 & 80.56 & 80.55 \\
\hline 18 & 24.65 & 24.32 & 24.34 & 24.45 & 24.45 \\
\hline 19 & 25.14 & 24.94 & 24.94 & 25.06 & 25.06 \\
\hline 20 & 25.39 & 25.01 & 25.05 & 25.16 & 25.16 \\
\hline 21 & 66.15 & 66.86 & 66.83 & 66.71 & 66.72 \\
\hline 22 & 35.48 & 34.87 & 34.88 & 35.07 & 35.06 \\
\hline 23 & 60.28 & 59.88 & 59.89 & 60.04 & 60.05 \\
\hline 24 & 70.47 & 69.46 & 69.51 & 69.78 & 69.77 \\
\hline 25 & 75.01 & 74.96 & 74.94 & 74.9 & 74.89 \\
\hline 26 & 75.66 & 75.48 & 75.51 & 75.53 & 75.53 \\
\hline 27 & 25.06 & 24.85 & 24.85 & 24.97 & 24.97 \\
\hline 28 & 24.58 & 24.29 & 24.31 & 24.42 & 24.42 \\
\hline 29 & 25.3 & 25.07 & 25.08 & 25.21 & 25.2 \\
\hline 32 & 88.76 & 88.6 & 88.64 & 88.63 & 88.63 \\
\hline 33 & 27.28 & 26.77 & 26.8 & 27 & 26.99 \\
\hline 35 & 18.33 & 18.56 & 18.55 & 18.52 & 18.52 \\
\hline 36 & 84.51 & 85.52 & 85.42 & 84.97 & 84.97 \\
\hline 37 & 115.85 & 116.52 & 116.52 & 115.88 & 115.87 \\
\hline 38 & 27.37 & 26.68 & 26.76 & 27.17 & 27.17 \\
\hline 39 & 113.59 & 114.88 & 114.8 & 114.13 & 114.13 \\
\hline 40 & 114.15 & 115.28 & 115.25 & 114.66 & 114.67 \\
\hline 41 & 28.45 & 28.23 & 28.23 & 28.47 & 28.47 \\
\hline 42 & 119.84 & 120.29 & 120.28 & 119.85 & 119.84 \\
\hline 43 & 28.94 & 28.33 & 28.36 & 28.7 & 28.7 \\
\hline 44 & 28.55 & 28.1 & 28.12 & 28.39 & 28.39 \\
\hline 45 & 28.24 & 28.07 & 28.07 & 28.27 & 28.27 \\
\hline 46 & 89.18 & 90.29 & 90.19 & 89.71 & 89.71 \\
\hline 47 & 116.67 & 117.26 & 117.26 & 116.65 & 116.65 \\
\hline 48 & 27.37 & 26.53 & 26.63 & 27.14 & 27.14 \\
\hline
\end{tabular}


Table 3 - continued from previous page

\begin{tabular}{cccccc} 
ATOM N & VACUO & $\mathrm{H}_{2} \mathrm{O}$ & $\mathrm{MeOH}$ & $\mathrm{ACN}$ & DMSO \\
\hline 40 & 114.46 & 115.58 & 115.51 & 114.96 & 114.97 \\
50 & 28.34 & 28.14 & 28.14 & 28.37 & 28.37 \\
51 & 113.87 & 115.07 & 114.99 & 114.38 & 114.4 \\
52 & 120.56 & 120.93 & 120.99 & 120.58 & 120.57 \\
53 & 28.65 & 28.21 & 28.23 & 28.5 & 28.49 \\
54 & 28.8 & 28.16 & 28.2 & 28.53 & 28.52 \\
55 & 28.18 & 28.05 & 28.02 & 28.21 & 28.21 \\
59 & 30.52 & 29.9 & 29.85 & 30.2 & 30.2 \\
61 & 30.06 & 29.48 & 29.53 & 29.82 & 29.82 \\
63 & 30.44 & 29.85 & 29.89 & 30.23 & 30.24 \\
65 & 30.26 & 29.66 & 29.72 & 30.03 & 30.04 \\
67 & 30.11 & 29.55 & 29.6 & 29.88 & 29.88 \\
69 & 30.46 & 29.83 & 29.87 & 30.14 & 30.14 \\
70 & 125.83 & 126.59 & 126.59 & 126.28 & 126.29 \\
71 & 28.05 & 27.89 & 27.89 & 27.94 & 27.94 \\
72 & 28.24 & 28.25 & 28.24 & 28.27 & 28.26 \\
74 & 32.08 & 30.79 & 30.83 & 31.35 & 31.35 \\
75 & 125.92 & 126.6 & 126.58 & 126.33 & 126.33 \\
76 & 27.98 & 27.81 & 27.82 & 27.86 & 27.86 \\
77 & 28.2 & 28.17 & 28.17 & 28.2 & 28.2 \\
79 & 32.05 & 30.79 & 30.83 & 31.34 & 31.34 \\
80 & 24.96 & 24.88 & 24.89 & 24.92 & 24.92 \\
81 & 25.38 & 25.24 & 25.24 & 25.36 & 25.36
\end{tabular}


Table 4: Vibrational modes of C1: $v$, stretching; $\delta, \gamma$ in-plane and out-plane bending, respectively; $\tau$, torsion; superscript: $\mathrm{Ph}$, aromatic ring vibrations; $\mathrm{A}$, vibrations connected with 'enolic' part of the molecule(left); B, vibrations connected with 'keto' part of the molecule(right).

$V_{\mathrm{IR}} \exp _{\mathrm{Ass}} v_{\mathrm{IR}} \mathrm{Calc}_{\mathrm{A}} \mathrm{Calc}_{\mathrm{R}} \mathrm{km} \cdot \mathrm{mol}-1$

\begin{tabular}{|c|c|c|c|c|c|}
\hline & & 601 & 23.75 & $v^{\mathrm{cC}}$ & $\gamma_{\mathrm{COC}}$ \\
\hline 605 & $\gamma_{\mathrm{COH}} 605$ & & 142.03 & & $\gamma_{\mathrm{COH}}$ \\
\hline & & 622 & 5.09 & & \\
\hline
\end{tabular}

$623 \quad 23.69$

$628 \quad 9.09$

$644 \quad 40.35$

$646 \quad 173.64$

$663 \quad \delta_{\mathrm{ccc}}$

$\delta^{\mathrm{Ph}}$

$\gamma_{\mathrm{ccc}}$

$\gamma_{\mathrm{CCC}}^{\mathrm{Ph}}$

$\mid \begin{array}{ll}678 & 1.19 \\ 682 & 2.06 \\ 683 & 12.31\end{array}$

$\mid \begin{array}{ll}678 & 1.19 \\ 682 & 2.06 \\ 683 & 12.31\end{array}$

$693 \quad 0.42$

725

732
Assignment

$\delta_{\mathrm{CCC}}$

$\delta_{\text {CCC }}^{\mathrm{Ph}}$

$\gamma_{\mathrm{CCC}}^{\mathrm{PhB}}$

$\tau \mathrm{CCOH}$

$\tau \mathrm{CCOH}$

$\delta_{\mathrm{cc}}=\mathrm{C}$

$\delta_{\text {CCC }}^{\mathrm{PhA}}$

$\gamma_{\mathrm{CCC}}^{\mathrm{S}}$

$\gamma_{\mathrm{CCC}}^{\mathrm{S}}$

$\gamma^{\mathrm{Ph}}{ }_{\mathrm{B}}$

$\mathrm{C}=\mathrm{CH}$

$\gamma_{\mathrm{c}}=\mathrm{C}-\mathrm{OH}$

$\gamma_{\mathrm{C}=\mathrm{CH}}^{\mathrm{ph}}$

$\gamma_{\mathrm{CCC}}^{\mathrm{PhA}}$

$\gamma_{\mathrm{CCC}}^{\mathrm{PhA}}$ 
Table 4 - continued from previous page

1

2

5

6

7

8

12

13

14

15

16

17

18

19

20

21

22

23

24

25

26

27

28

29

30

31

32

33

34

35

36

37

38

39

40

41

42

43

44

45

46

47

48

49

50

51

52

53

54

55

56

57

58

59

60
Assignment

$v_{\mathbb{R}} \exp _{\mathrm{Ass}} v_{\mathrm{IR}} \mathrm{Calc}_{\mathrm{A}} \mathrm{Calc}_{\mathrm{IR}} \mathrm{km} \cdot \mathrm{mol}-1$

$\gamma_{\mathrm{CCC}}^{\mathrm{Ph}}$

734

$\gamma_{\mathrm{CCC}} \mathrm{Ph}$

$\delta_{\mathrm{CCC}}^{\mathrm{Ph}}$

$\delta_{\mathrm{CCC}}$

$\gamma_{\mathrm{CCH}}$

747

3.92

$\gamma_{\mathrm{CCC}}^{\mathrm{PhB}}$

$\gamma_{\mathrm{C} \_\mathrm{CH}}$

$754 \quad 8.52$

$\delta^{\mathrm{Ph}}{ }_{\mathrm{A}}$

$\mathrm{CCC}$

$\delta^{\mathrm{P}}{ }_{\mathrm{B}}$

$\mathrm{CCC}$

$\delta_{\mathrm{CCO}}^{\mathrm{K}}$

$\gamma_{\mathrm{C}=\mathrm{CH}}$

$774 \quad 11.89$

$795 \gamma_{\mathrm{CCC}} \mathrm{Ph}$

$804 \quad 66.38$

$810 \quad \gamma_{\mathrm{CCH}}^{\mathrm{Ph}}$

$812 \quad 22.66$

$815 \quad 1.49$

$829 \quad 22.98$

$\gamma_{\mathrm{CCC}}^{\mathrm{PhB}}$

$\gamma_{\mathrm{CCC}}^{\mathrm{PhA}}$

$\gamma^{\mathrm{Ph}}$

$\gamma^{\mathrm{Ph}}$

$\mathrm{CCH}$

$\gamma^{\mathrm{Ph}}{ }_{\mathrm{BCH}}$

$\gamma_{\mathrm{CCH}}^{\mathrm{PhA}}$

$\gamma_{\mathrm{CCH}}^{\mathrm{PhB}}$

$\gamma_{\mathrm{CCH}}^{\mathrm{PhA}}$

$833 \quad 10.35$

$\begin{array}{ll}852 & 3.77\end{array}$
$\delta_{\mathrm{cc}}=\mathrm{C}$

$\gamma^{\mathrm{Ph}}$

$\mathrm{CCH}$

$\gamma_{\mathrm{B}}^{\mathrm{Ph}}$

$\delta_{\mathrm{CC}=\mathrm{O}}^{\mathrm{K}}$ 
1

2

3

4

5

6

7

8

9

10

11

12

13

14

15

16

17

18

19

20

21

22

23

24

25

26

27

28

29

30

31

32

33

34

35

36

37

38

39

40

41

42

43

44

45

46

47

48

49

50

51

52

53

54

55

56

57

58

59

60
Table 4 - continued from previous page

$v_{\mathrm{IR}} \exp _{\mathrm{Ass}} v_{\mathrm{IR}} \mathrm{Calc}_{\mathrm{A}} \mathrm{Calc}_{\mathrm{IR}} \mathrm{km} \cdot \mathrm{mol}-1$

Assignment

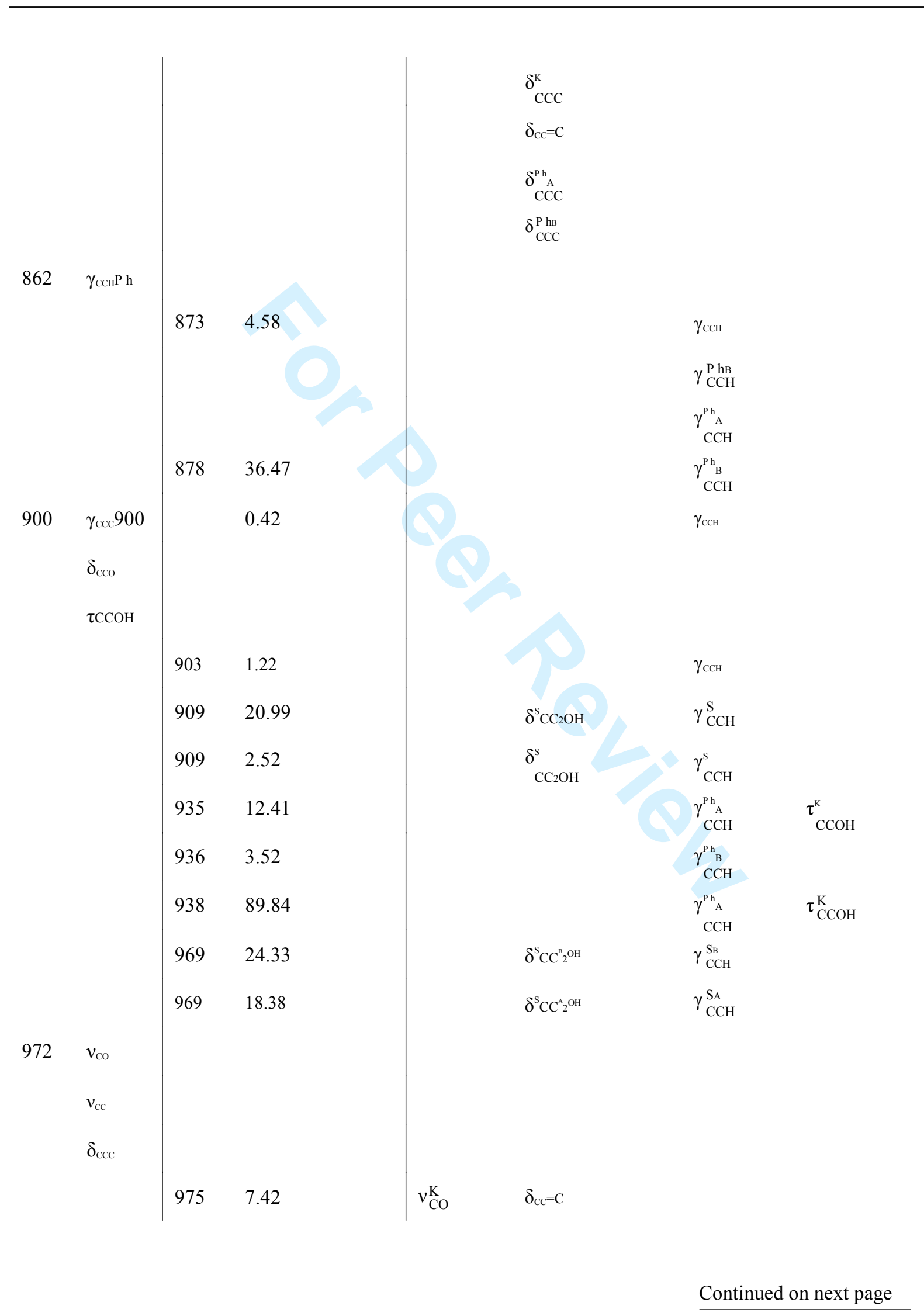

http://mc.manuscriptcentral..com/poc 
Table 4 - continued from previous page

$v_{\mathbb{R}} \exp _{A s s} v_{\mathbb{R}} \mathrm{Calc}_{\mathrm{A}} \mathrm{Calc}_{\mathbb{R}} \mathrm{km} \cdot \mathrm{mol}-1 \quad$ Assignment

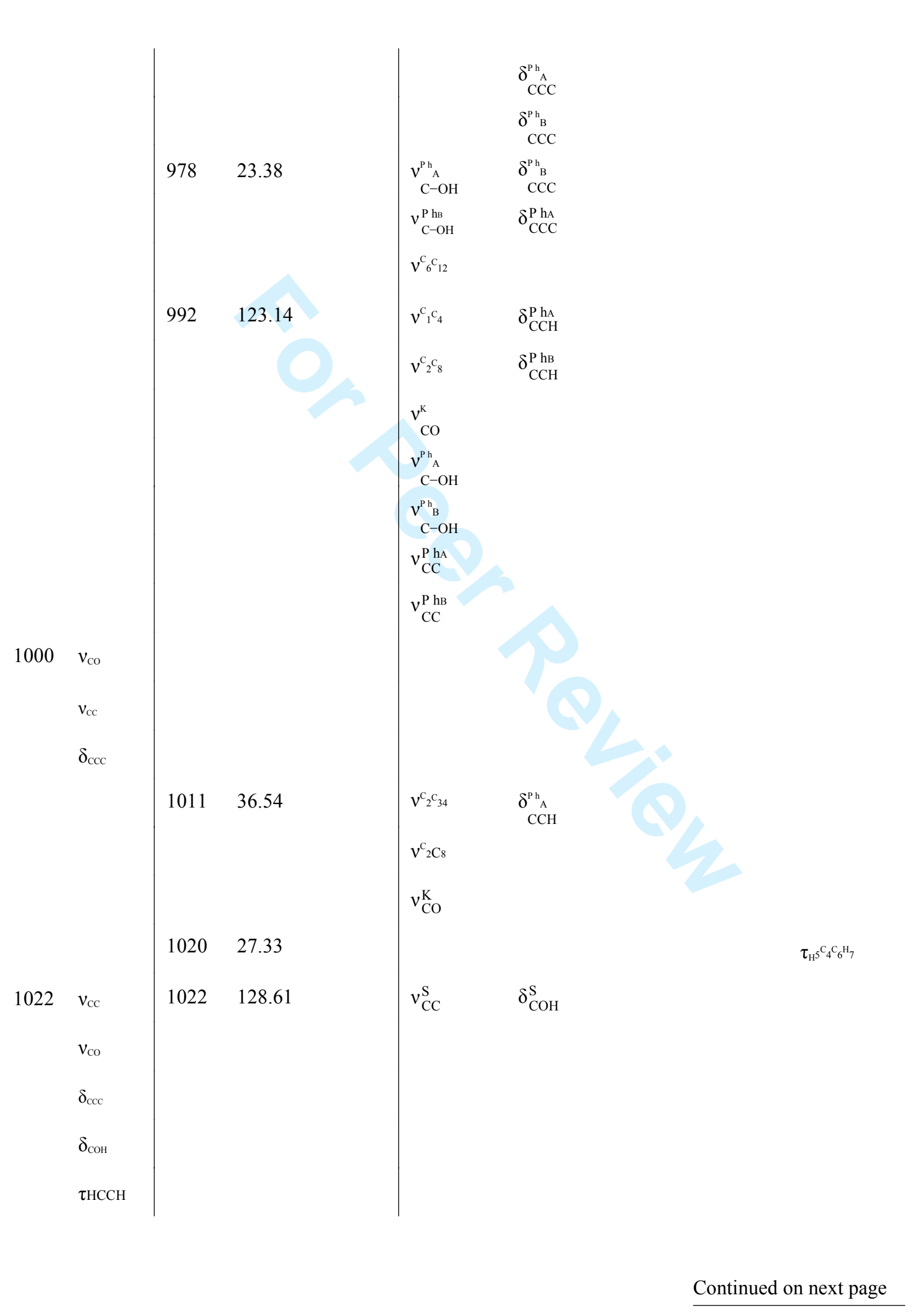

http://mc.manuscriptcentral.com/poc 
1

2

3

4

5

6

7

8

9

10

11

12

13

14

15

16

17

18

19

20

21

22

23

24

25

26

27

28

29

30

31

32

33

34

35

36

37

38

39

40

41

42

43

44

45

46

47

48

49

50

51

52

53

54

55

56

57

58

59

60

Table 4 - continued from previous page

$v_{\mathrm{IR}} \exp _{\mathrm{Ass}} v_{\mathrm{IR}} \mathrm{Calc}_{\mathrm{A}} \mathrm{Calc}_{\mathrm{IR}} \mathrm{km} \cdot \mathrm{mol}-1$

Assignment

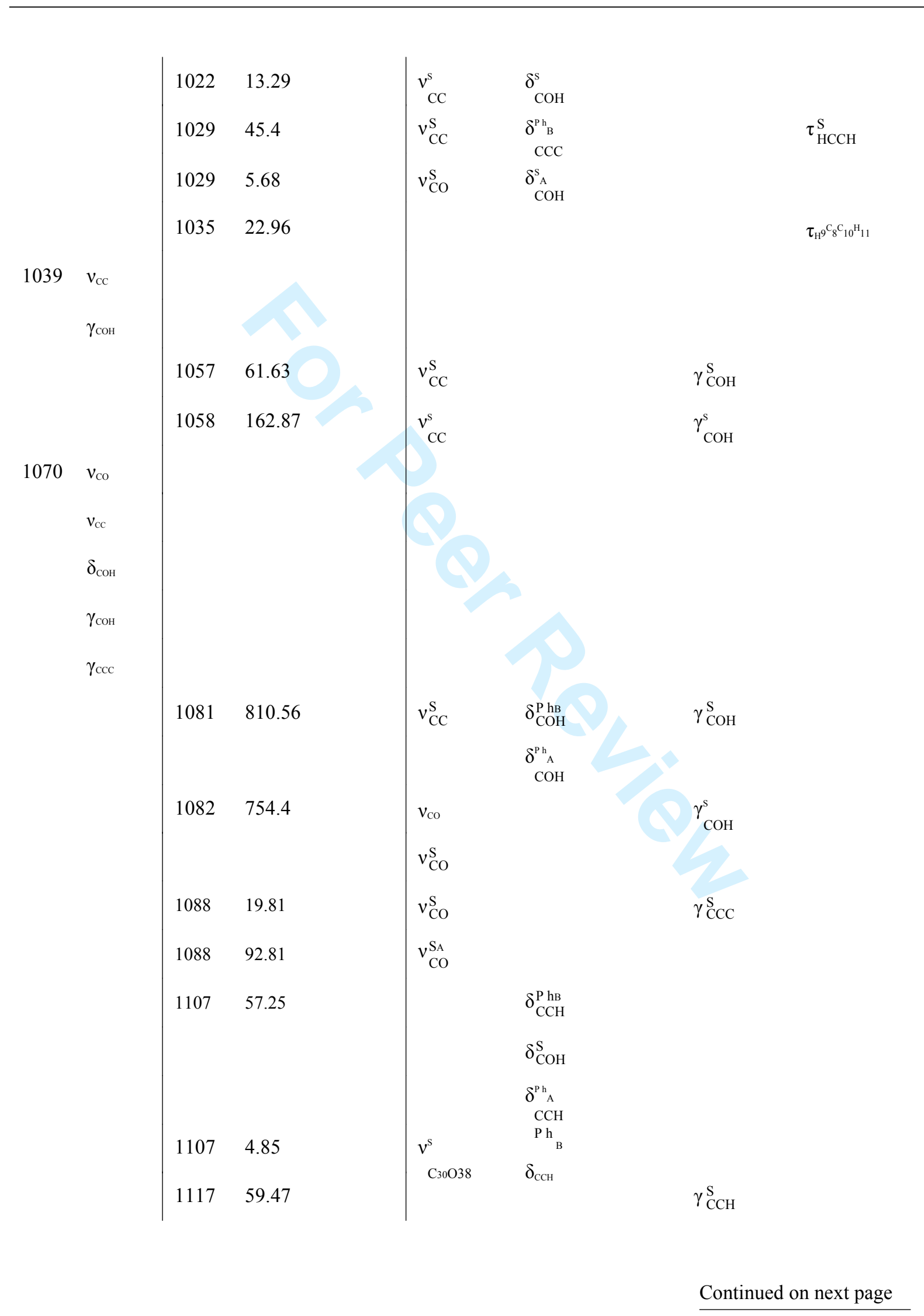

http://mc.manuscriptcentral.com/poc 
Table 4 - continued from previous page

$V_{\mathbb{R}} \exp _{A s s} v_{\mathbb{R}} \mathrm{Calc}_{\mathrm{A}} \mathrm{Calc}_{\mathbb{R}} \mathrm{km} \cdot \mathrm{mol}-1 \quad$ Assignment

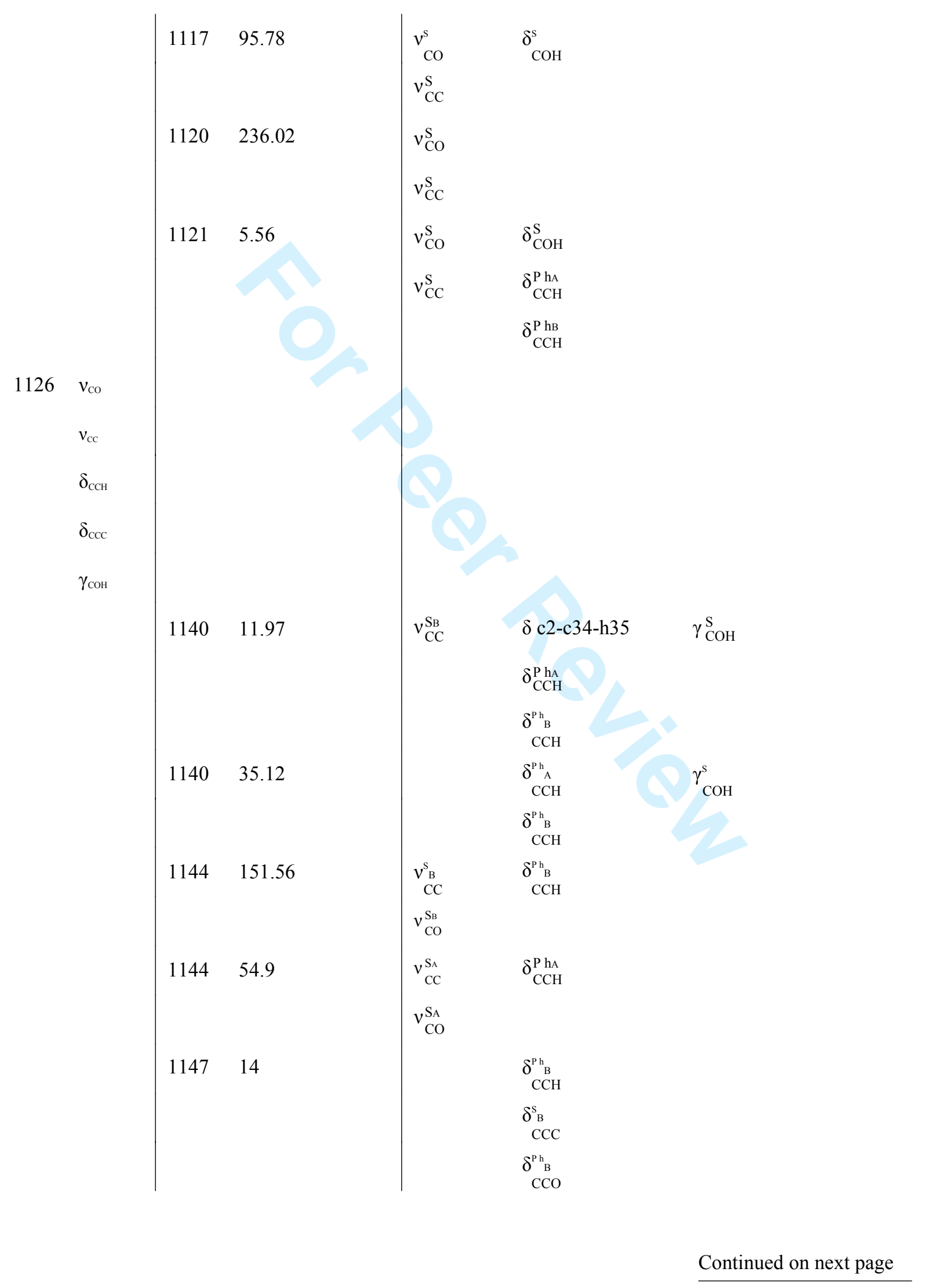

http://mc.manuscriptcentral.com/poc 
1

2

3

4

5

6

7

8

10

11

12

13

15

16

17

18

19

20

21

22

23

24

25

26

27

28

29

30

31

32

33

34

35

36

37

38

39

40

41

42

43

44

45

46

47

48

49

50

51

52

53

54

55

56

57

58

59

60
Table 4 - continued from previous page

$v_{\mathbb{R}} \exp _{A s s} v_{\mathbb{R}} \mathrm{Calc}_{\mathrm{A}} \mathrm{Calc}_{\mathrm{R}} \mathrm{km} \cdot \mathrm{mol}-1$

Assignment

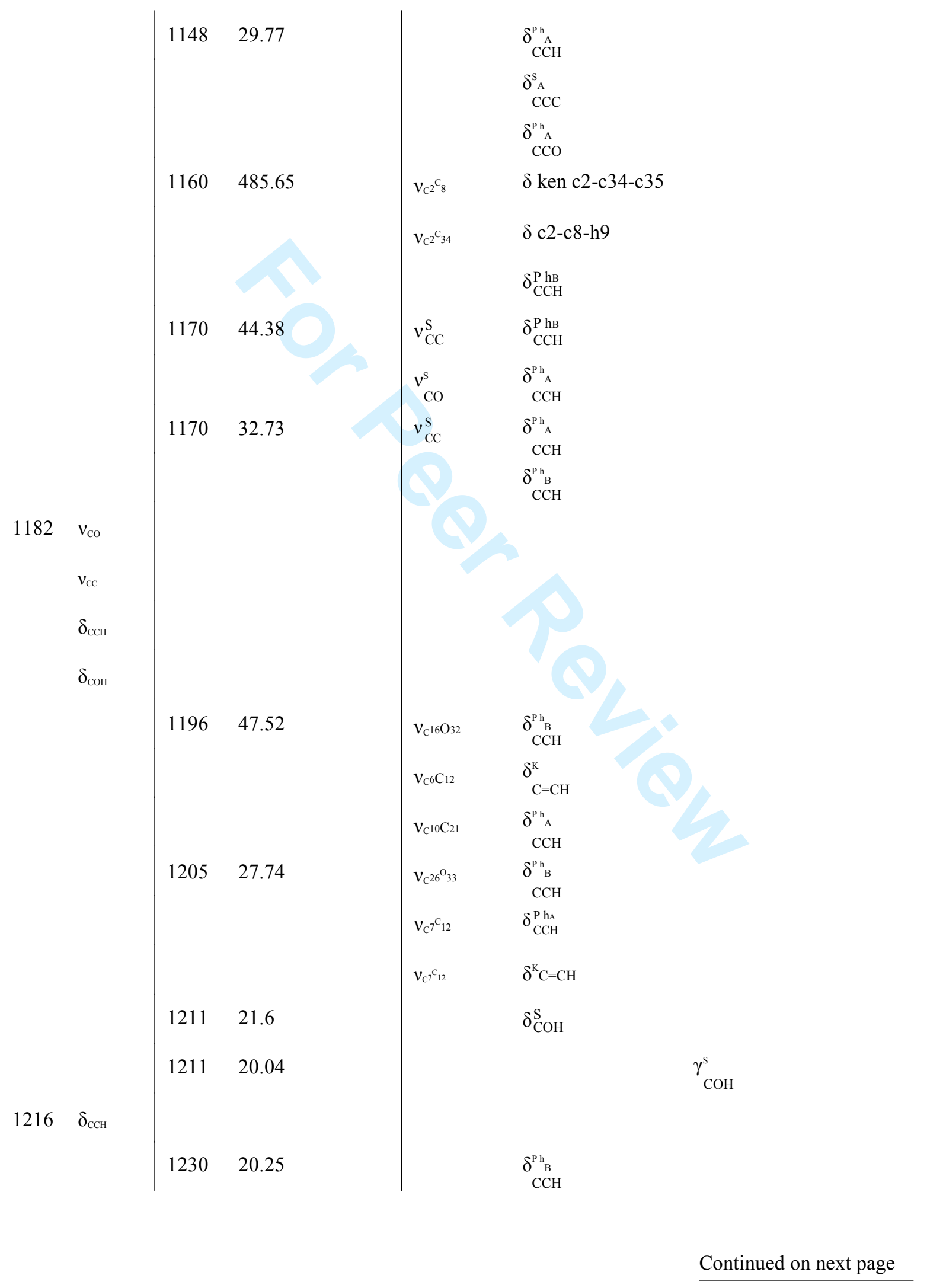

http://mc.manuscriptcentral.com/poc 
Table 4 - continued from previous page

$v_{\mathrm{IR}} \exp _{\mathrm{Ass}} v_{\mathrm{IR}} \mathrm{Calc}_{\mathrm{A}} \mathrm{Calc}_{\mathrm{IR}} \mathrm{km} \cdot \mathrm{mol}-1$

Assignment

2

4

5

6

8

12

13

14

15

16

17

18

19

20

21

22

23

24

25

26

27

28

29

30

31

32

33

34

35

36

37

38

39

40

41

42

43

44

45

46

47

48

49

50

51

52

53

54

55

56

57

58

59

60

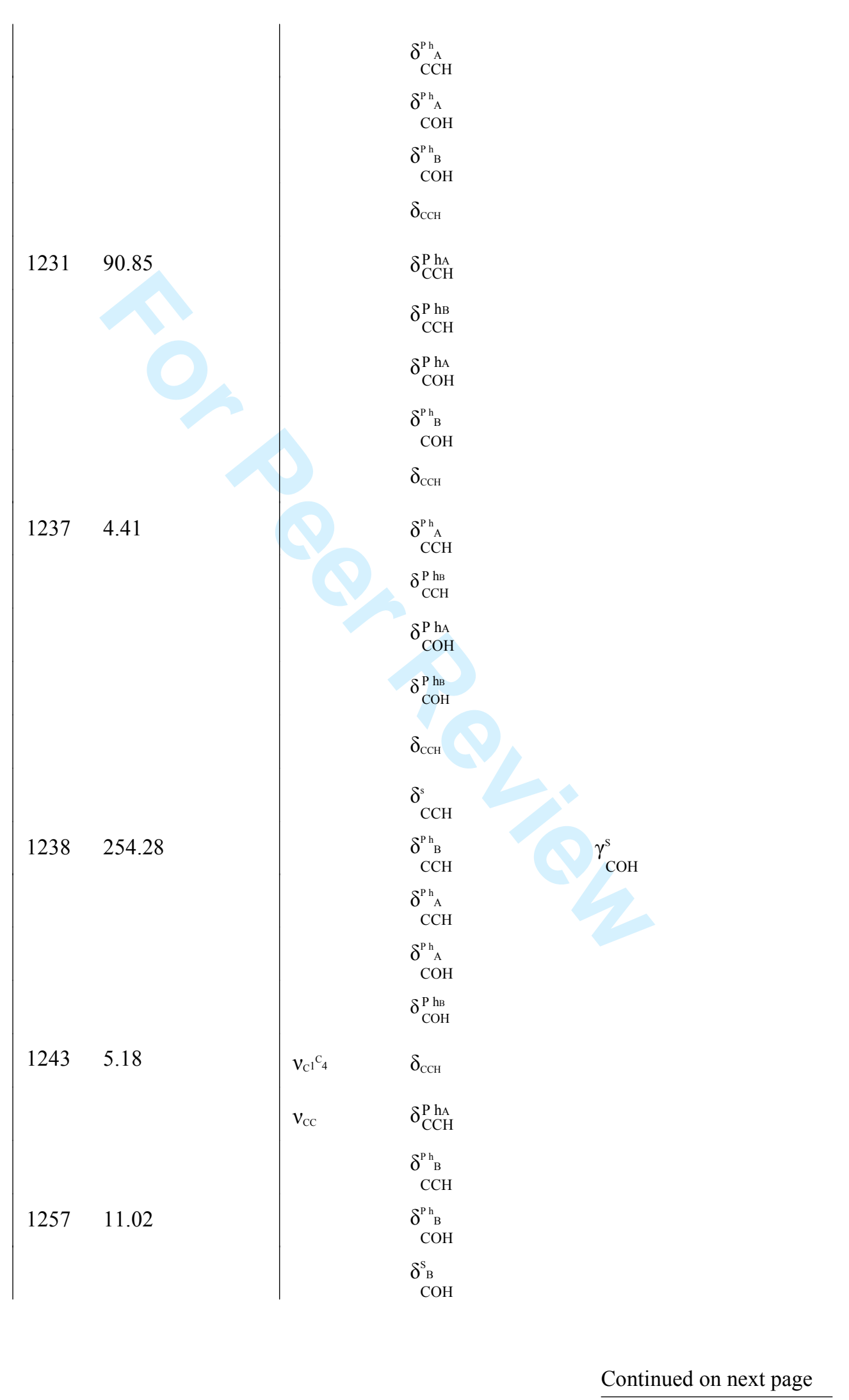

http://mc.manuscriptcentral.com/poc 
1

2

3

4

5

6

7

8

9

10

11

12

13

14

15

16

17

18

19

20

21

22

23

24

25

26

27

28

29

30

31

32

33

34

35

36

37

38

39

40

41

42

43

44

45

46

47

48

49

50

51

52

53

54

55

56

57

58

59

60
Table 4 - continued from previous page

$v_{\mathrm{IR}} \exp _{\mathrm{Ass}} v_{\mathrm{IR}} \mathrm{Calc}_{\mathrm{A}} \mathrm{Calc}_{\mathrm{IR}} \mathrm{km} \cdot \mathrm{mol}-1$

Assignment

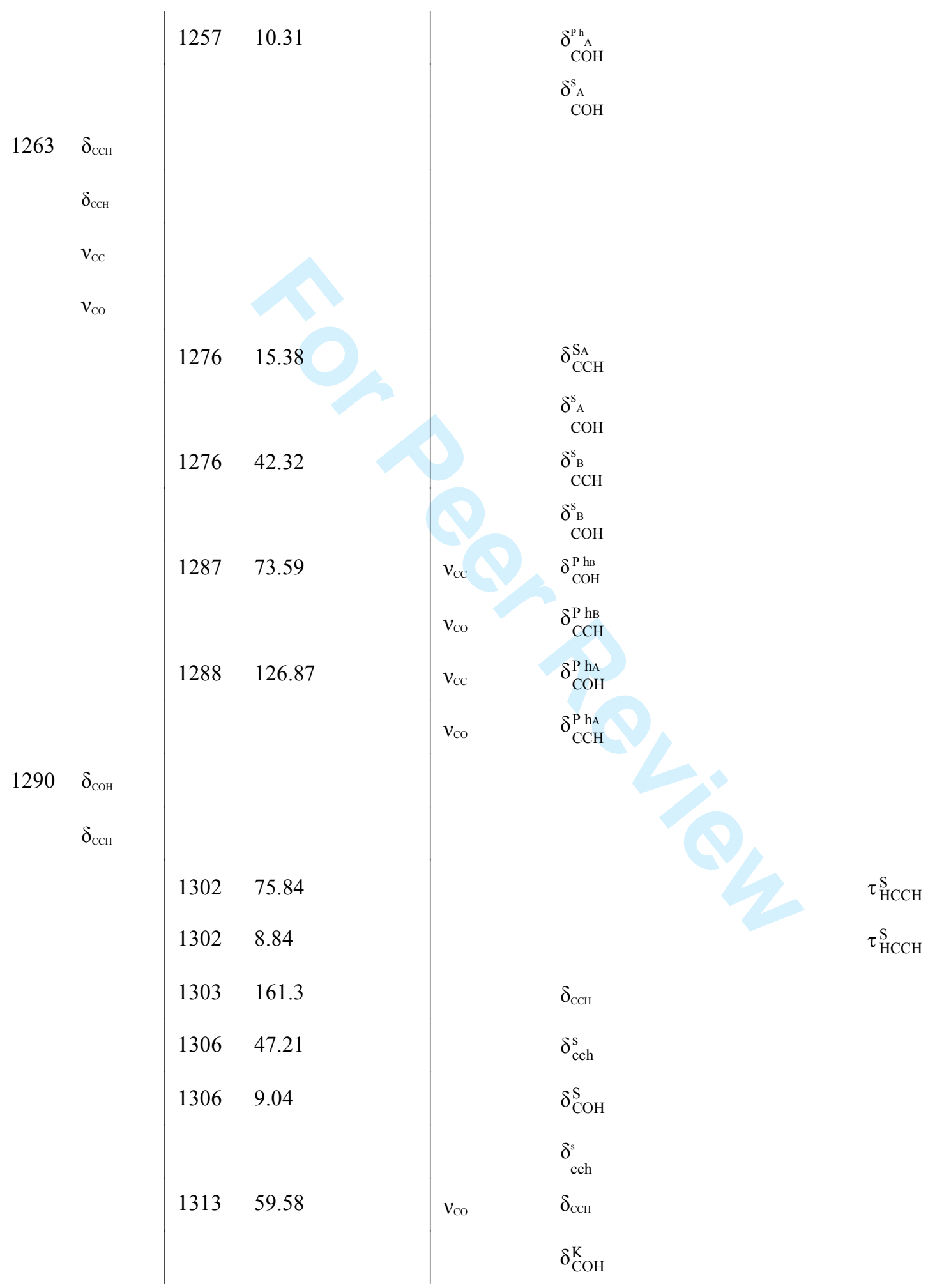

Continued on next page 
Table 4 - continued from previous page

$v_{\mathrm{IR}} \exp _{\mathrm{Ass}} v_{\mathrm{IR}} \mathrm{Calc}_{\mathrm{A}} \mathrm{Calc}_{\mathrm{IR}} \mathrm{km} \cdot \mathrm{mol}-1$

Assignment

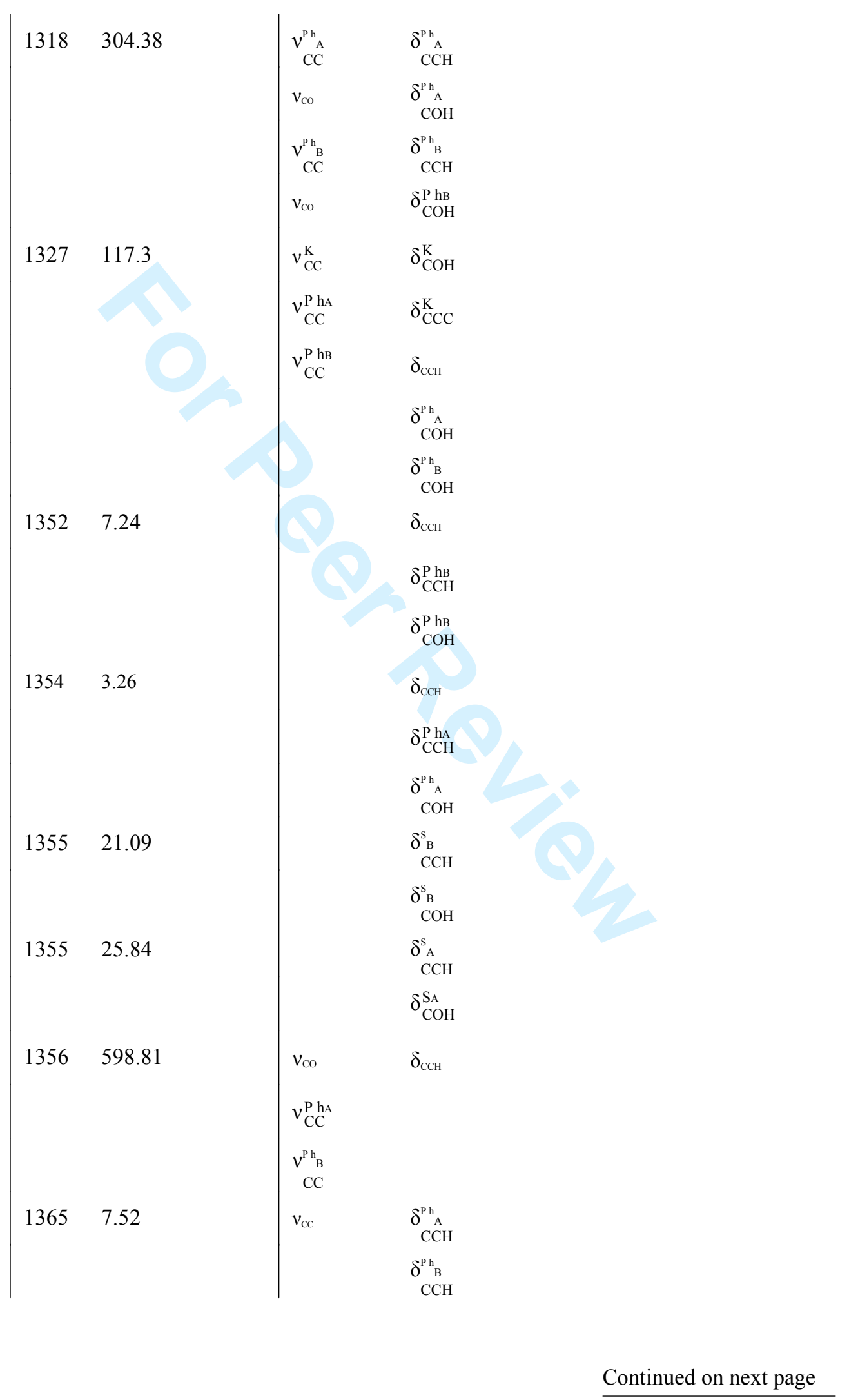

http://mc.manuscriptcentral.com/poc 
1

2

3

4

5

6

7

8

9

10

11

12

13

14

15

16

17

18

19

20

21

22

23

24

25

26

27

28

29

30

31

32

33

34

35

36

37

38

39

40

41

42

43

44

45

46

47

48

49

50

51

52

53

54

55

56

57

58

59

60
Table 4 - continued from previous page

$v_{\mathbb{R}} \exp _{A s s} v_{\mathbb{R}} \mathrm{Calc}_{\mathrm{A}} \mathrm{Calc}_{\mathrm{RR}} \mathrm{km} \cdot \mathrm{mol}-1$

Assignment

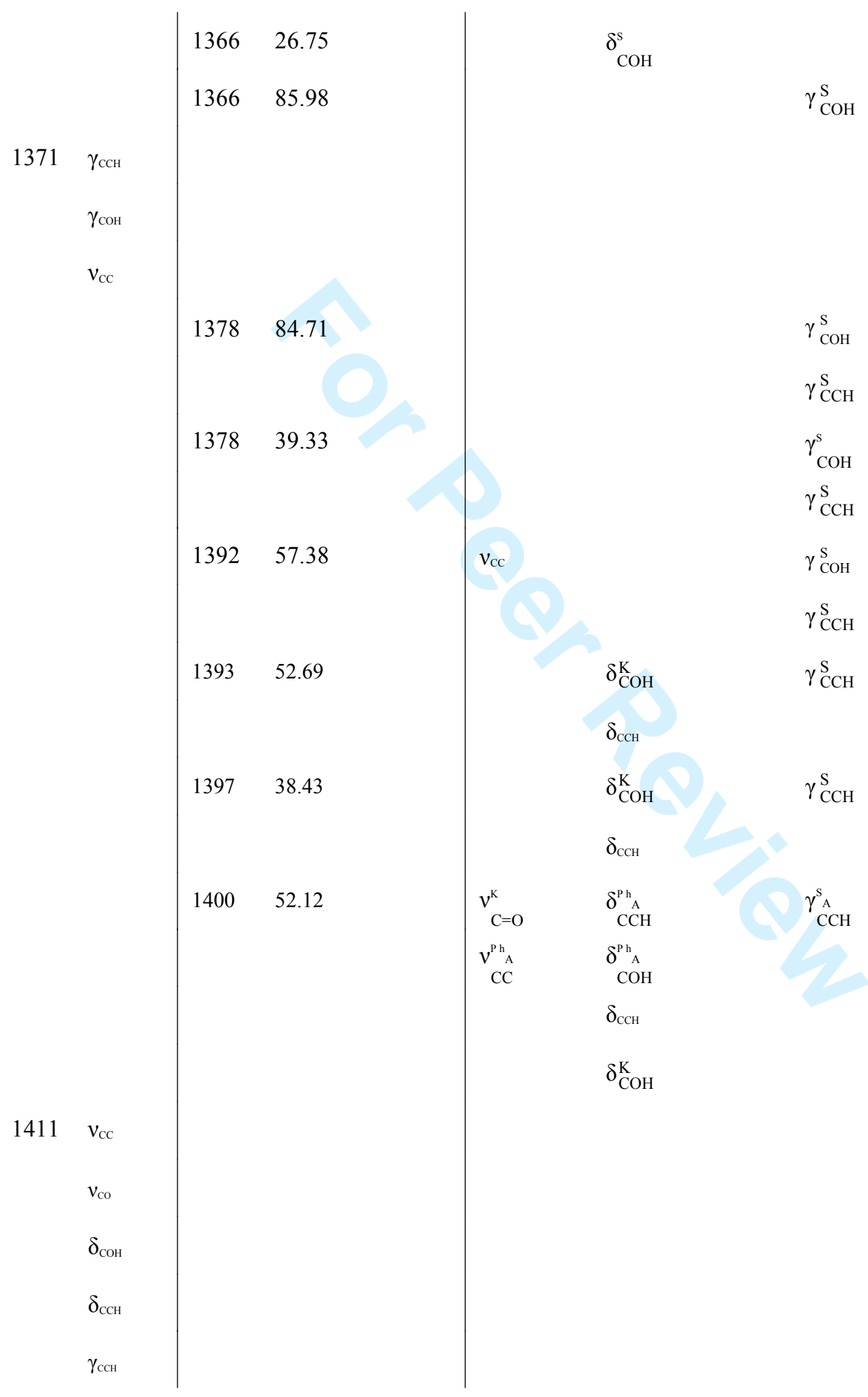

Continued on next page 
Table 4 - continued from previous page

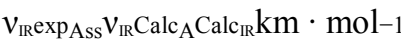

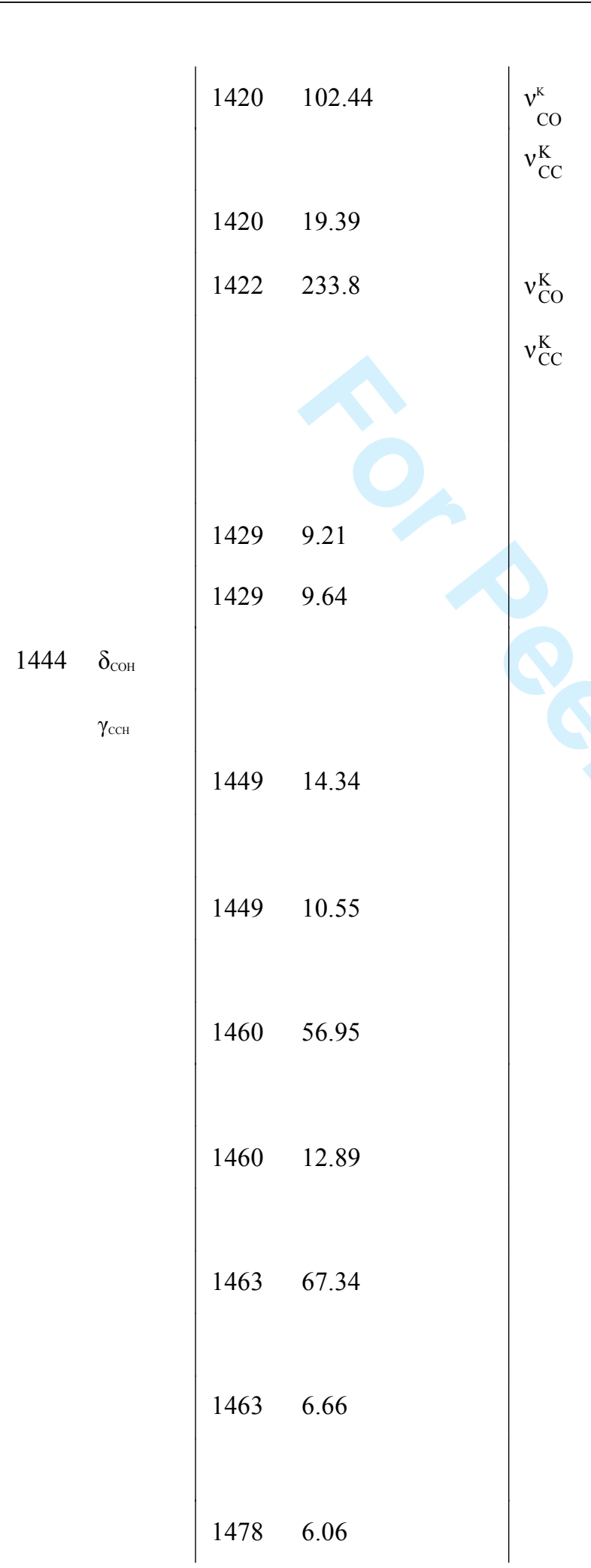

Assignment

$\begin{array}{cc}\delta_{\mathrm{COH}}^{\mathrm{K}} & \gamma_{\mathrm{CCH}}^{\mathrm{s}} \\ & \\ & \gamma_{\mathrm{CCH}}^{\mathrm{s}} \\ & \gamma_{\mathrm{CCH}}^{\mathrm{SB}} \\ \delta_{\mathrm{COH}}^{\mathrm{K}} & \\ \delta_{\mathrm{CCH}}^{\mathrm{K}} & \\ \delta_{\mathrm{CCH}} & \\ \delta_{\mathrm{COH}}^{\mathrm{Ph}} & \\ \delta^{\mathrm{ph}} & \\ { }_{\mathrm{COH}}^{\mathrm{CH}} & \gamma_{\mathrm{CCH}}^{\mathrm{s}} \\ \delta_{\mathrm{COH}}^{\mathrm{ph}} & \gamma_{\mathrm{B}}^{\mathrm{s}} \\ \mathrm{COH}^{\mathrm{s}} & { }_{\mathrm{CCH}}\end{array}$

$\delta_{\mathrm{COH}}^{\mathrm{PhA}}$

$\gamma_{\mathrm{CCH}}^{\mathrm{SA}}$

$\delta_{\mathrm{COH}}^{\mathrm{PhB}}$

$\gamma_{\mathrm{CCH}}^{\mathrm{SB}}$

$\delta_{\mathrm{COH}}^{\mathrm{PhA}}$

$\gamma_{\mathrm{CCH}}^{\mathrm{SA}}$

$\delta^{\mathrm{Ph}}{ }_{\mathrm{B}}$

$\mathrm{COH}$

$\delta^{\mathrm{Ph}}{ }_{\mathrm{A}}$

$\mathrm{COH}$

$\delta^{\mathrm{ph}}{ }^{\mathrm{B}}$

$\mathrm{COH}$

$\delta^{\mathrm{ph}}{ }_{\mathrm{A}}$

$\mathrm{COH}$

$\delta_{\mathrm{COH}}^{\mathrm{PhB}}$

$\delta_{\mathrm{COH}}^{\mathrm{PhA}}$

$\gamma^{\mathrm{S}}$

${ }^{\mathrm{CCH}}$

$\gamma_{\mathrm{A}}^{\mathrm{S}}$

$\mathrm{CCH}$

$\gamma_{\mathrm{B}}^{\mathrm{s}}$

$\mathrm{CCH}$

$\gamma_{\mathrm{CCH}}^{\mathrm{s}}$

$\gamma_{\mathrm{CCH}}^{\mathrm{SB}}$

$\gamma_{\mathrm{CCH}}^{\mathrm{SA}}$

$\delta_{\mathrm{COH}}^{\mathrm{PhB}}$

$\gamma_{\mathrm{CCH}}^{\mathrm{SB}}$

$\delta^{\mathrm{Ph}}{ }_{\mathrm{A}}$

$\mathrm{COH}$

$\gamma_{\mathrm{A}}^{\mathrm{s}}$

$\delta^{\mathrm{ph}}{ }_{\mathrm{B}}$

$\mathrm{COH}$

$\delta^{\mathrm{Ph}}{ }_{\mathrm{A}}$

$\mathrm{COH}$
$\mathrm{CCH}$

$\gamma_{\text {B }}^{\mathrm{s}}$

$\mathrm{CCH}$

$\gamma_{\mathrm{CCH}}^{\mathrm{s}}$ 
1

2

3

4

5

6

7

10

11

12

13

14

15

16

17

18

19

20

21

22

23

24

25

26

27

28

29

30

31

32

33

34

35

36

37

38

39

40

41

42

43

44

45

46

47

48

49

50

51

52

53

54

55

56

57

58

59

60

Table 4 - continued from previous page

$v_{\mathbb{R}} \exp _{A s s} v_{\mathbb{R}} \mathrm{Calc}_{\mathrm{A}} \mathrm{Calc}_{\mathrm{R}} \mathrm{km} \cdot \mathrm{mol}-1$

Assignment

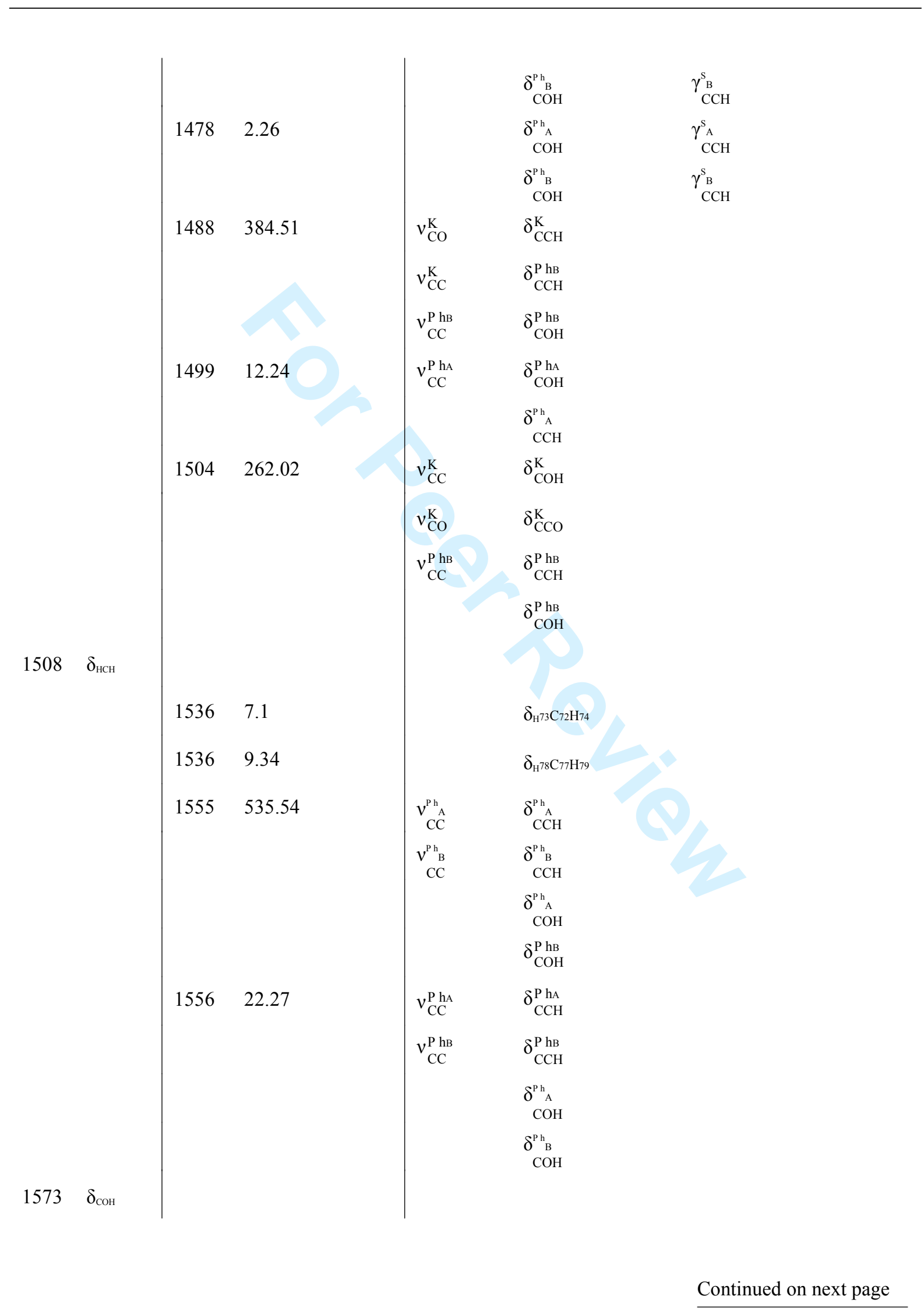

http://mc.manuscriptcentral..com/poc 
Table 4 - continued from previous page

$v_{\mathrm{IR}} \exp _{\mathrm{Ass}} v_{\mathrm{IR}} \mathrm{Calc}_{\mathrm{A}} \mathrm{Calc}_{\mathrm{IR}} \mathrm{km} \cdot \mathrm{mol}-1$

Assignment

2
3
4
5
6
7
8
9

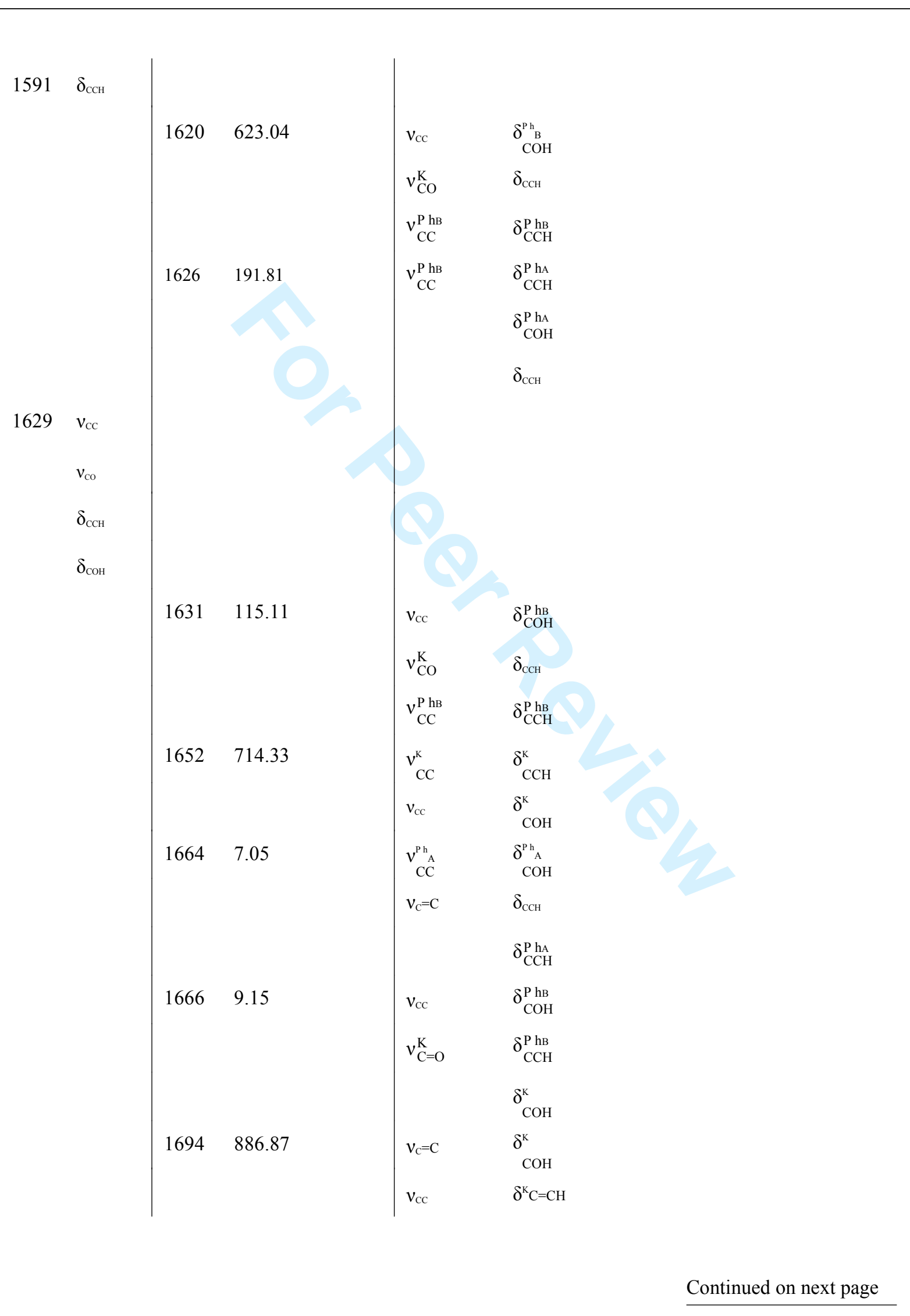

http://mc.manuscriptcentral.com/poc 
1

2

3

4

5

6

7

8

9

10

11

12

13

14

15

16

17

18

19

20

21

22

23

24

25

26

27

28

29

30

31

32

33

34

35

36

37

38

39

40

41

42

43

44

45

46

47

48

49

50

51

52

53

54

55

56

57

58

59

60
Table 4 - continued from previous page

$v_{\mathrm{IR}} \exp _{\mathrm{Ass}} v_{\mathrm{IR}} \mathrm{Calc}_{\mathrm{A}} \mathrm{Calc}_{\mathrm{IR}} \mathrm{km} \cdot \mathrm{mol}-1$

Assignment

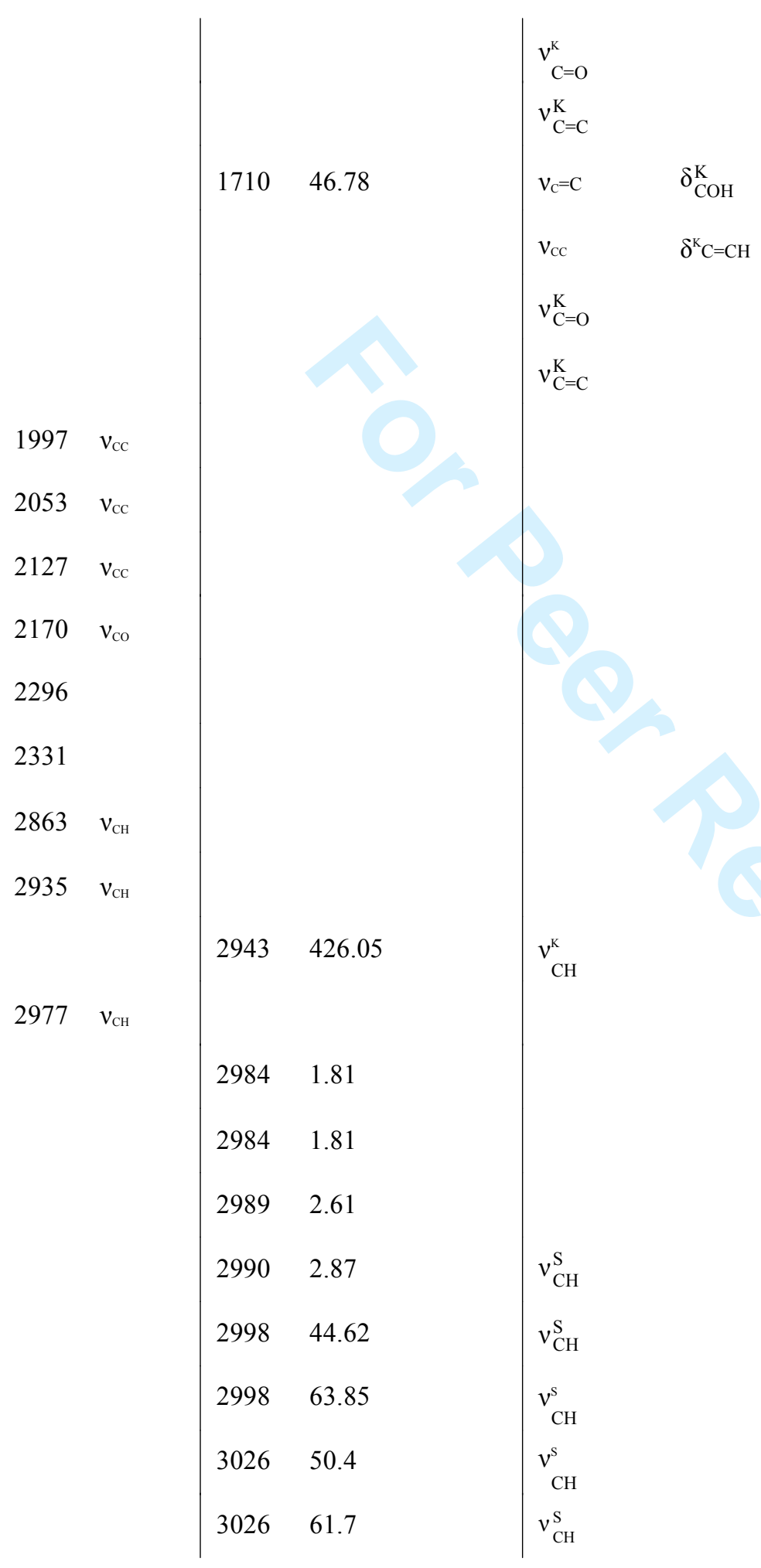


Table 4 - continued from previous page

$v_{\mathrm{IR}} \exp _{\mathrm{Ass}} v_{\mathrm{IR}} \mathrm{Calc}_{\mathrm{A}} \mathrm{Calc}_{\mathrm{IR}} \mathrm{km} \cdot \mathrm{mol}-1$

Assignment

\begin{tabular}{|ll|l}
3046 & 26.37 \\
3046 & 27.24 \\
3073 & 32.19 \\
3073 & 22.72 \\
3077 & 35.93 \\
3077 & 50.39 \\
3177 & 0.1 & $v_{\mathrm{CH}}^{\mathrm{s}}$ \\
3186 & 12.25 \\
3188 & 0.11 & $v_{\mathrm{CH}}^{\mathrm{S}}$ \\
3197 & 11.89 \\
3207 & 3.83 \\
$v_{\mathrm{CH}}^{\mathrm{S}}$ \\
$v_{\mathrm{CH}}^{\mathrm{S}}$ \\
3207 & 3.48 \\
3209 & 6.39 \\
3209 & 5.41 \\
3222 & 4.73 & $v_{\mathrm{CH}}^{\mathrm{S}}$ \\
3222 & 1.11 & $v_{\mathrm{CH}}^{\mathrm{S}}$ \\
3223 & 18.14 \\
3567 & 947.75 \\
3569 & 589.9 \\
3724 & 54.31 & 67.51 \\
$v_{\mathrm{CH}}$ \\
$v_{\mathrm{CH}}$ \\
$v_{\mathrm{CH}}$ \\
$v_{\mathrm{CH}}$ \\
$v_{\mathrm{CH}}^{\mathrm{Ph}}$ \\
$v_{\mathrm{CH}}^{\mathrm{Ph}}$
\end{tabular}

Continued on next page

http://mc.manuscriptcentral.com/poc 
1

2

3

4

5

6

7

8

9

10

11

12

13

14

15

16

17

18

19

20

21

22

23

24

25

26

27

28

29

30

31

32

33

34

35

36

37

38

39

40

41

42

43

44

45

46

47

48

49

50

51

52

53

54

55

56

57

58

59

60
Table 4 - continued from previous page

$v_{\mathrm{IR}} \exp _{\mathrm{Ass}} v_{\mathrm{IR}} \mathrm{Calc}_{\mathrm{A}} \mathrm{Calc}_{\mathrm{IR}} \mathrm{km} \cdot \mathrm{mol}-1$

Assignment

$\mid$\begin{tabular}{ll|l}
3742 & 59.96 & $v_{\mathrm{OH}}^{\mathrm{s}}$ \\
3766 & 24.08 & $v_{\mathrm{OH}}^{\mathrm{s}}$ \\
3766 & 32.37 & $v_{\mathrm{OH}}^{\mathrm{S}}$ \\
\hline
\end{tabular}




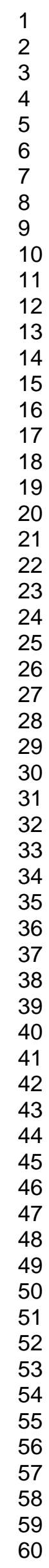




\title{
How glucosylation triggers physical-chemical properties of Curcumin: an experimental and theoretical study
}

\author{
Rois Benassi ${ }^{*}$, Erika Ferrari, Sandra Lazzari, Francesca Pignedoli, Ferdinando Spagnolo, Monica \\ Saladini \\ Department of Chemistry, University of Modena and Reggio Emilia, via Campi 183, 41100 \\ Modena, Italy
}

\begin{abstract}
In the present study we investigate the structures of glucosylated curcumin derivatives with DFT at B3LYP/6-31G* level. A conformational analysis is performed in order to determine the GS (conformational minimum) and TS (rotational transition state) of curcumin derivatives and then their electronic features are evaluated. HOMO and LUMO frontier orbitals and Maps of Electron Density Potential (MEPs) are plotted and compared. In order to correlate their predicted spectroscopic properties with IR, UV-vis and NMR experimental data we extended the theoretical study on electronic properties to different solvents $\left(\mathrm{H}_{2} \mathrm{O}, \mathrm{MeOH}, \mathrm{ACN}, \mathrm{DMSO}\right)$. The main finding is that the curcuminic core maintains the same geometrical and electronic structures in all compounds miming the metal coordination capability showed by curcumin. Therefore we may confirm that the presence of glucose does not affect the electronic properties of the derivatives.

Keywords: Curcumin, DFT, $\beta$-keto-enolic structure, glucosyl-curcuminoids
\end{abstract}

*Corresponding author: tel +39 0592055046, Fax +39 059373543, e-mail: rois.benassi@ unimore.it 


\section{Introduction}

Curcumin (1,7-bis(4-hydroxy-3-methoxyphenyl)-1,6-heptadiene-3,5-dione), a yellow spice extracted from Curcuma Longa L. rhizomes, is used in a wide range of applications, from industrial dyes to pharmaceutical treatments. ${ }^{[1-3]}$ It is proved that curcumin holds selective metal-chelating properties that are pharmaceutically interesting. ${ }^{[4]}$ In the field of medicinal chemistry one of the most promising properties of curcumin is its metal ligating ability towards Gallium and Iron. ${ }^{[5,6]}$ This feature can be exploited for a variety of pharmaceutical aims like metal overload detoxification, metal delivery and radio imaging. Despite these potential pharmaceutical applications, curcumin has a low water solubility and limited bioavailability that makes it difficult to handle for pharmaceutical use. To improve chemical properties of curcumin, several derivatives were synthesized and studied by means of theoretical and experimental data. ${ }^{[7,8]}$ The glucosylation of the aromatic ring was found to enhance curcumin water-solubility and kinetic stability which is a fundamental feature for drug bioavailability. ${ }^{[9]}$ The compounds were characterized and their ability to act as metal-chelating agents was also evaluated. ${ }^{[9,10]}$ Biological properties of these molecules were also tested and they showed cytotoxicity towards human ovarian carcinoma cell line leading to an improvement of Cisplatin efficiency with higher selectivity towards cancer than non-cancer cells. ${ }^{[11]}$ In order to elucidate chemical-physical properties of these molecules and to correlate their electronic structures with the ability to act as metal chelating agents, in the present study we employ DFT calculations for a conformational analysis of the compounds reported in Figure 1. A full optimization of the geometry is followed by a conformational search in order to study the potential energy surface (PES) as a function of the rotation of the O-C exocyclic dihedral angle $(\phi)$ (Figure 1). It was reported that the presence of different solvents perturbs intra- or inter-molecular hydrogen bonds in curcumin modifying its photophysical behavior. ${ }^{[12]}$ Therefore now we perform also a theoretical study on the electronic properties of glucosyl-curcuminoids in different solvents in order to correlate their predicted spectroscopic properties with IR, UV-vis and NMR experimental data. 


\section{Methods}

\section{$2.1 \quad$ Computational details}

The computations of all the studied compounds were performed by the DFT approach. The structures were fully optimized using hybrid-functional B3LYP applied to $6-31 \mathrm{G}^{*}$ basis set $\left(\mathrm{B} 3 \mathrm{LYP} / 6-31 \mathrm{G}^{*}\right)^{[13-15]}$ by means of Gaussian $03^{[16]}$ package of programs.

In a previous study we described curcumin structure using both B3LYP/6-311G** and B3LYP/6-31G* levels. ${ }^{[17]}$ By comparing the obtained results, B3LYP/6-31G* showed to be a good compromise between accuracy and precision. All the calculated properties agreed well with those obtained using B3LYP/6-311G**. Therefore we decided to employ B3LYP/6-31G* in order to study the influence of glucosylation on curcumin properties. GaussView $03^{[18]}$ was used as a plotting tool for data visualization.

Thermodynamics were obtained from vibrational analysis employing general procedures. The analysis of the calculated vibrational properties always confirmed the conformational minimum (GS) or rotational transition state (TS), characterized as a saddle point, for the considered structure.

Atom charges were calculated from the optimized geometries at B3LYP/6-31G* level with the CHELPG approach as implemented in Gaussian 03. The molecular electrostatic potential maps (MEPs) were plotted by Gaussview and reported onto 0.02 e/Bohr isosurface of electrondensity; representations of HOMO and LUMO orbital density were referred to an isovalue of 0.0004 .

The solvent effects were evaluated by employing the self-consistent reaction field (SCRF) method with polarized continuum model (PCM). ${ }^{[19-21]}$

The absorption wavelengths and oscillator strengths were calculated by means of timedependent density functional theory (TD-DFT) as implemented in Gaussian 03. The magnetic isotropic shielding tensors $(\sigma)$ for ${ }^{1} \mathrm{H}$ and ${ }^{13} \mathrm{C}$ NMR were calculated using the standard GIAO 
(Gauge-Independent Atomic Orbital) ${ }^{[22]}$ at B3LYP/6-31G* approach with the Gaussian 03 program package.

\subsection{Conformational analysis}

Starting with the mono glucosyl-compounds, Series A (Figure 1), all structures were fully optimized with the DFT approach at B3LYP/6-31G* level.

We utilized the geometries of the full reoptimized structures as the starting point for a rigid PES scan of the O-C exocyclic dihedral angle $(\phi)$ (Figure 1). The dihedral angle $\phi$ was rotated at $15^{\circ}$ step size up to a complete $360^{\circ}$ turn.

Minimum and maximum points on rigid PES were optimized and characterized as GS and TS states from the vibrational analysis. For all structures the starting point was confirmed to be the most stable structure.

Geometry of compounds C (Figure 1) was built from the more stable structure of the corresponding series A, adding a second glucose molecule. All the possible dispositions of the second sugar molecule with respect to curcumin planar skeleton were examined and optimized. Calculations on $\mathbf{C}$ compounds were then run using the same approach (rigid PES search of $\phi$ dihedral angle, optimization of the obtained structures, vibrational analysis, CHELPG charges calculation, plots of HOMO and LUMO molecular orbitals and MEPs).

\subsection{Spectroscopy}

Spectroscopic data were collected only for previously synthesized compounds $\mathbf{C} .^{[10]}$

NMR spectra were recorded at $300 \mathrm{~K}$ on a Bruker Avance AMX-400 spectrometer with a Broad Band $5 \mathrm{~mm}$ probe (inverse detection). Nominal frequencies were $100.13 \mathrm{MHz}$ for ${ }^{13} \mathrm{C}$ and 400.13 MHz for ${ }^{1} \mathrm{H}$. The typical acquisition parameters for ${ }^{1} \mathrm{H}$ were as follows: $20 \mathrm{ppm}$ spectral bandwidth $(\mathrm{SW}), 6.1 \mu$ s pulse width $\left(90^{\circ}\right.$ pulse hard pulse on $\left.{ }^{1} \mathrm{H}\right), 0.5-1$ s pulse delay, $216-512$ 


\section{Results and discussion \\ 3.1. Conformational analysis}

Previous studies ${ }^{[7,17,26]}$ demonstrated that the keto-enolic tautomer of curcumin is the most stable form, so that we decided to use it to describe the core of all curcumin derivatives reported in Figure 1.

The first remarkable result is that for all compounds the curcuminic core maintains a completely planar conformation with the same geometrical parameters as curcumin. ${ }^{[17]}$ allowing Curcumin-like electronic conjugation of aromatic/ $\pi$ electrons. This was found to be a fundamental feature to allow metal coordination through dissociated enolic moiety. ${ }^{[27]}$ The introduction of a glucose molecule may perturb curcumin skeleton by means of hydrogen bond interactions or conjugation effects, therefore we decided to scan the dihedral angle $\phi$ as it defines the sugar 
molecule orientation. Table 1 reports energies, thermodynamic quantities and dihedral angles $(\phi, \theta)$ values obtained from conformational analysis of all studied compounds.

A plot of $\Delta \mathrm{G}$ values as a function of $\phi$ angle for $\mathbf{A}$ type compounds is reported in Figure 2. From the analysis of rotational free energy, two states of rotational maximum $\left(\mathrm{TS}_{1}\right.$ and $\left.\mathrm{TS}_{2}\right)$ and two states of minimum $\left(\mathrm{GS}_{1}\right.$ and $\left.\mathrm{GS}_{2}\right)$ are found.

The main differences in $\phi$ values are observed for the absolute minimum conformation $\left(\mathrm{GS}_{1}\right)$, ranging from $71^{\circ}$ to $118^{\circ}$ (Table 1). The $\phi$ value increases following the decrease of aromatic substituent steric hindrance $\left(\mathrm{H}<\mathrm{OH}<\mathrm{OCH}_{3}\right) . \mathrm{GS}_{2}$ ground state is less influenced by substituent bulkiness, since the sugar moiety is rotated thus minimizing the interaction with the substituent on the aromatic ring (Figure 3). As a consequence, $\phi$ values in $\mathrm{GS}_{2}$ are similar, ranging from $282^{\circ}$ to $293^{\circ}$.

Free activation energies of rotation $\left(\Delta \mathrm{G}^{*}\right)$ of $\mathrm{TS}_{1}$ for $\mathbf{A 2}$ and $\mathbf{A 3}$ are similar (A2 $7.67 \mathrm{kcal}$ $\mathrm{mol}^{-1}$; A3 $7.45 \mathrm{kcal} \mathrm{mol}^{-1}$ ), while for $\mathbf{A 1} \Delta \mathrm{G}^{*}$ assumes an higher value $\left(9.43 \mathrm{kcal} \mathrm{mol}^{-1}\right)$. In A1 the geometrical analysis of $\mathrm{GS}_{1}$ (Figure 3) provides evidence for a hydrogen bond between $\mathrm{OH}$ phenolic group and a hydroxylic oxygen atom of the sugar moiety $(\mathrm{O} 33-\mathrm{H} 60 \cdots \mathrm{O} 491.96 \AA$, O-H $\cdots \mathrm{O}$ angle $158.6^{\circ}$ ). The hydrogen bond break in $\mathbf{A 1}$ strongly contributes to the higher $\Delta \mathrm{G}^{*}$. In $\mathbf{A 2}$ and A3 only steric effects are responsible for the $\Delta \mathrm{G}^{*}$ value and no evidence of significant $\mathrm{H}$-bond is observed. Previous study on similar $\beta$-diketo-mono-glucosylated derivatives ${ }^{[27]}$ showed the formation of a hydrogen bond involving $\mathrm{OCH}_{3}$ aromatic substituent and glucosydic moiety $(\mathrm{OH} \cdots \mathrm{O}$ $2.23 \AA$, OHO angle $155^{\circ}$ ). Instead in A2, no hydrogen interaction is observed suggesting that the greater extent of $\pi$ delocalization with respect to the previously studied compound, probably makes $\mathrm{OCH}_{3}$ group less capable to form a hydrogen bond.

In di-glucosylated compounds $\mathbf{C}$, all the possible dispositions of the two sugar moieties with respect to the planar skeleton of curcumin were optimized and the results for $\mathbf{C 2}$ are reported in 
Figure 4, $\mathrm{C1}$ and $\mathrm{C3}$ show the same trend. The syn-syn configuration is the most stable therefore it is used as the starting point for conformational analysis on $\phi$ dihedral angle. For all compounds $\mathbf{C}$ the value of the $\theta$ dihedral angle results close to the corresponding $\phi$ angle in $\mathrm{GS}_{1}$ state of compounds A with opposite sign.

Compounds $\mathbf{C}$ show the same trend as mono-glucosylated series A regarding PSE. The $\Delta \mathrm{G}^{*}$ and dihedral angles for $\mathbf{A}$ and $\mathbf{C}$ homologues are superimposable with a maximum difference of $\pm 2^{\circ}$ (Table 1).

For each compound $\mathbf{C}$, in all states of maximum and minimum, the value of the not scanned $\theta$ dihedral angle is constant and equal to the $\phi$ value obtained in the ground state $\left(\mathrm{GS}_{1}\right)$ with opposite sign. This evidence suggests that the two sugar molecules rotate independently and do not influence each other during the rotation. We suggest that $\theta$ angle can rotate with the same potential energy as $\phi$ since compounds $\mathbf{C}$ maintain the same symmetric structure observed in curcumin. For all compounds the relatively low $\Delta \mathrm{G}^{*}$ values $\left(<10.04 \mathrm{kcal} \mathrm{mol}^{-1}\right)$ allow a "free" rotation of the glucosydic group around $\phi$ and $\theta$ dihedral angle at room temperature. Anyway the free rotation of the glucose moieties does not exert any steric hindrance on keto-enolic group in any conformation, maintaining it accessible to a metal ion for the coordination reaction.

We observed also the formation of a hydrogen bond interaction between the $\mathrm{OH}$ phenolic

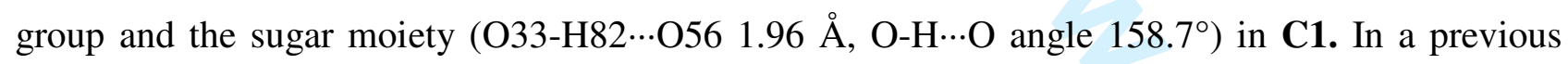
study on compounds $\mathbf{C}$ we discovered that the nature of a meta substituent on the aromatic ring affects its lipophilicity in the order $\mathrm{OH}>\mathrm{H}>\mathrm{OCH}_{3}{ }^{[10]}$ Today computational data may account for the unexpected increased lipophilicity of $\mathbf{C 1}$ due to the formation of an intra-molecular hydrogen bond with the glucosydic moiety. 


\subsection{Molecular Orbitals}

HOMO and LUMO molecular orbitals were calculated for both GS and TS conformers for A and $\mathbf{C}$ series. Figure $\mathbf{5}$ shows HOMO and LUMO molecular orbitals at the $\mathrm{GS}_{1}$ conformation, HOMO and LUMO for the other conformations $\mathrm{GS}_{2}, \mathrm{TS}_{1}, \mathrm{TS}_{2}$ are perfectly overlapping with $\mathrm{GS}_{1}$. HOMO and LUMO are delocalized through the whole curcuminic core, allocating the electron density both on the dienic and benzenic moieties as found for curcumin. ${ }^{[17,26]}$ The introduction of one or two glucose molecules does not add any interaction between the newly attached substituent and the aromatic groups, thus not affecting the peculiar conjugation of aromatic/ $\pi$ electrons of curcumin core confirming the results of conformational analysis.

\subsection{MEPs}

Figure 6 shows the MEPs of $\mathrm{GS}_{1}$ states. In all compounds, the negative charge of the curcuminic core is located on the $\beta$-keto-enolic oxygen atoms, resembling the same trend of curcumin. ${ }^{[17,26]}$ Table 2 reports charge density values of $\beta$-keto-enolic and phenolic oxygen atoms of $\mathbf{A 1}$ and $\mathbf{C 1}$ in $\mathrm{GS}_{1}$ state. By comparing the values obtained in vacuum with those of curcumin we can observe variation of $\approx 0.004$ in the negative charge of the $\beta$-keto-enolic oxygen atoms, thus suggesting the same potential metal coordination ability of curcumin. ${ }^{[6]}$ The presence of OH groups in A1 and C1 introduces a further center of negative charge that might affect their physicalchemical properties. Although enolic and phenolic oxygens show almost the same negative charge values the involvement of phenolic oxygen in metal coordination is prevented by the higher $\mathrm{pK}_{\mathrm{a}}$ value $\left[\mathrm{pK}_{\mathrm{a}}\right.$ (phenolic) 9.4; $\mathrm{pK}_{\mathrm{a}}$ (enolic) 8.2$] .{ }^{[11]}$ The negative charge on $\mathrm{OH}$ aromatic substituent evidences its strong electron withdrawing ability, which probably influences the $\mathrm{pK}_{\mathrm{a}}$ value of the $\beta$ keto-enolic group. In fact $\mathbf{C 1}$ was found to be the most acidic compound in aqueous solution and the more effective $\mathrm{Fe}(\mathrm{III})$ and $\mathrm{Ga}(\mathrm{III})$ chelating agent. ${ }^{[11]}$

Compounds $\mathbf{A}$ and $\mathbf{C}$ show the same electronic properties (MEPs, CHELPG) suggesting similar coordination ability. Therefore the analysis of solvent effects is limited to $\mathbf{C}$ compounds in 
order to compare their electronic properties with experimental data. MEPs calculated in different solvents look like those in vacuum; the corresponding density charge values are reported in Table 2.

We can observe a general increase in the negative charge on ketonic oxygen atom in solvents with respect to vacuum. This effect seems to be directly related to the solvent polarity. In fact $\mathrm{H}_{2} \mathrm{O}$ gives the major increase in negative charge. On the contrary the ability to form hydrogen bonds seems not to influence charge distribution. The high charge density on the enolic function may account for the replacement of hydrogen atom by metal ion with a lower $\mathrm{pK}_{\mathrm{a}}$ value with respect to free ligand system, enabling metal coordination and preventing hydroxide precipitation.

MEPs do not take into account higher electrostatic effects than polarization which may be very important in metal complexation. Anyway the higher charge density on keto-enolic group with respect to sugar oxygen atoms suggests that the keto-enolic function is the only group potentially involved in metal coordination. In addition to the chelating effect, the enolic proton acidity enforces this assumption. Previous NMR study on compounds $\mathbf{C}^{[9]}$ established that sugar moiety was never involved in metal ligation as no ${ }^{1} \mathrm{H}$ and ${ }^{13} \mathrm{C}$ chemical shifts were observed upon metal addition.

\subsection{NMR spectroscopy}

${ }^{1} \mathrm{H}$ and ${ }^{13} \mathrm{C}$ NMR spectra of compounds $\mathbf{C}$ were recorded and compared with the calculated values. Figure 7 reports ${ }^{1} \mathrm{H}$ NMR spectrum of $\mathbf{C 1}$ in DMSO- $d_{6}$. Experimental data evidence only one set of signals corresponding to the keto-enolic form; the complete symmetry $\left(\mathrm{C}_{2 \mathrm{v}}\right)$ of the molecule, due to resonance between the two possible keto-enolic limiting structures, is supported by the equivalence of double bonds and aromatic rings, and by ${ }^{13} \mathrm{C} \mathrm{NMR}$ spectrum giving undistinguishable and equivalent peaks for ketonic and enolic carbons. $\mathbf{C 2}$ and $\mathbf{C 3}$ behave similarly. The fit between experimental chemical shifts of glucosyl-curcuminoids in DMSO and MeOD for both ${ }^{1} \mathrm{H}$ (Figure 8A) and ${ }^{13} \mathrm{C}$ (Figure 9B) illustrates the very small variations due to experimental conditions. 
The GIAO (Gauge-Indipendent-Atomic-Orbitals) isotropic magnetic shielding tensors $\left(\sigma_{\text {calc }}\right)$ were calculated for $\mathbf{C 1}, \mathbf{C 2}$ and $\mathbf{C 3}$ in vacuum, $\mathrm{H}_{2} \mathrm{O}, \mathrm{MeOH}$, and DMSO (Table 1-3, supplementary material). Bearing in mind the experimental molecular symmetry of glucosyl-curcuminoids, the average value of $\sigma_{\text {calc }}\left(\bar{\sigma}_{\text {calc }}\right)$ between the two keto-enolic limit structures was calculated and reported in Table 3 for each solvent system.

${ }^{13} \mathrm{C}$ and ${ }^{1} \mathrm{H}$ experimental chemical shift assignments were supported by $2 \mathrm{D}$ COSY, HSQC and HMBC experiments. The data are reported in Table 4 and Table 5.

Plotting the experimental ${ }^{13} \mathrm{C}$ and ${ }^{1} \mathrm{H}$ chemical shifts $\left(\delta_{\exp }\right)$ in MeOD and DMSO versus the $\bar{\sigma}_{\text {calc }}$ in vacuum or in the same solvent system, a linear regression is observed: $\delta_{\exp }=\mathrm{a} \cdot \bar{\sigma}_{\text {calc }}+\mathrm{b}\left(\mathrm{a}, \mathrm{b}\right.$ and $\mathrm{R}^{2}$ parameters are given in Table 4 and Table 5.). This relationship is then used to predict the chemical shifts $\left(\delta_{\text {pred }}\right) \cdot{ }^{[17,28]}$ As already observed, ${ }^{[29]}$ the correlation between experimental chemical shifts and calculated isotropic screening constants is better for ${ }^{13} \mathrm{C}$ than for ${ }^{1} \mathrm{H}$. In fact the correlation coefficient $\mathrm{R}^{2}$ ranges from 0.9834 to 0.9939 for ${ }^{13} \mathrm{C}$ and from 0,9186 to 0.9718 for ${ }^{1} \mathrm{H}$.

A comparison with previously reported Curcumin predicted ${ }^{13} \mathrm{C}$ NMR data ${ }^{[18]}$ highlights how for glucosyl-curcuminoids the presence of the sugar moiety experiences the solvent effect; in fact linear regressions are definitely better when calculated on the basis of $\bar{\sigma}_{\text {calc }}$ in the same solvent system than in vacuum.

${ }^{1} \mathrm{H} \delta_{\text {pred }}$ values (Table 5) provide good correlation with $\delta_{\exp }$ with exception of $\mathrm{H}-1$, due to the fact that the GIAO $\sigma_{\text {calc }}$ has almost the same value in the two resonance limit structures of ketoenolic moiety. As a consequence electronic delocalization cannot be predicted accurately. Table 5 data confirm the importance of the solvent environment in predicting correct ${ }^{1} \mathrm{H} \delta_{\text {pred; }}$ in fact the gap in $\mathrm{R}^{2}$ value between in vacuum and solvent prediction is higher for ${ }^{1} \mathrm{H}(0.0117-0.0319)$ than ${ }^{13} \mathrm{C}$ (0.0003-0.0044). 
$\Delta \mathrm{R}^{2}$ values $\left(\mathrm{R}_{\text {solvent }}^{2}{ }^{-} \mathrm{R}^{2}\right.$ vacuum $)$ prove that the solvent has a greater effect in $\mathbf{C} 1$ and $\mathbf{C 2}$ than in C3; ${ }^{1} \mathrm{H}$ NMR data of $\mathbf{C 1}$ are better predicted in $\mathrm{MeOH}$ than in DMSO, $\mathbf{C 3}$ behaves in the opposite way while for $\mathbf{C 2}$ the two solvents show the same effect. This behavior suggests that the $\mathrm{OH}$ and $\mathrm{OCH}_{3}$ groups in $\mathbf{C 1}$ and $\mathbf{C 2}$ respectively may interact with the solvent through hydrogen bond and/or dipolar interactions.

Figure 9 reports the plotting of ${ }^{1} \mathrm{H}$ and ${ }^{13} \mathrm{C} \delta_{\exp }$ vs. $\bar{\sigma}_{\text {calc }}$ for $\mathbf{C} \mathbf{1}$ together with the linear relationships. $\mathbf{C 1}$ behaves differently from $\mathbf{C 2}$ and $\mathbf{C 3}$ as it concerns anomeric proton (H-11), in fact this proton is not well predicted by calculation as a consequence of the intra-molecular hydrogen bond between the $\mathrm{OH}$ phenolic group and the sugar moiety.

\subsection{UV-Vis spectroscopy}

Each compound shows a strong intense absorption band in the 300-500 nm wavelength region. Absorption maxima are reported in Table 6 together with calculated values in the corresponding solvent. The red shift in DMSO with respect to ACN medium follows the order C1 $>\mathbf{C 2}>\mathbf{C 3}$ and it is related to the higher polarity of the phenolic substituent $\left(\mathrm{OH}>\mathrm{OCH}_{3}>\mathrm{H}\right)$ interacting with different polar solvents. A solvent dependent red shift in maximum was found also in curcumin ${ }^{[12]}$ and was related to solvent polarity and proticity. In fact methanol, a strong hydrogen bond-donating as well as hydrogen bond accepting solvent gave the greatest maximum red shift. In our case the three compounds in $\mathrm{H}_{2} \mathrm{O}$ and $\mathrm{MeOH}$ behaves similarly, probably because the hydrogen bond effect is masked by the presence of sugar moiety. The same trend is observed in the predicted values.

\subsection{Infra Red Spectroscopy}

Usually the calculated harmonic vibrational wavenumbers are higher than the experimental ones, because of the anharmonicity, incomplete treatment of electron correlation and use of finite 
one-particle basis set. ${ }^{[29]}$ A linear relationship with a good correlation coefficient is obtained plotting the experimental wavenumbers versus the calculated ones $\left(\overline{\mathbf{v}}_{\text {calc }}\right)$ for all compounds $\mathbf{C}$; Figure 10 reports $\mathbf{C 1}$ data. This result suggests that the over-estimation of calculated wave numbers is quite systematic and allows to predict FT-IR spectra. The slope of the linear regression $(\mathrm{a}=$ 0.9991) is greater than the value (0.9613) estimated at B3LYP/6-31G* level for a great number of different compounds. ${ }^{[30]}$ A list of the experimental and calculated frequencies together with a tentative assignment is reported as supplementary material (Table 4 supplementary material)

\section{Conclusions}

We have demonstrated that the addition of bulky sugar groups does not affect the electronic properties of the curcuminic core, allowing metal coordination through keto-enolic moiety. A glucose moiety interacts only with meta-substituents on the aromatic ring influencing its lipophilicity.

The solvent effect is a critical aspect in predicting spectroscopic data which well agree with the experimental ones.

\section{Acknowledgments}

We are thankful to the "Consorzio Interuniversitario per il Calcolo Automatico dell'Italia Nord Orientale - CINECA" and to the "Laboratorio di Calcolo Scientifico Avanzato Interdipartimentale dell'Università degli Studi di Modena e Reggio Emilia” for computing facilities. We are grateful to the "Centro Interdipartimentale Grandi Strumenti - C.I.G.S." of the University of Modena and Reggio Emilia and to the "Fondazione Cassa di Risparmio di Modena" for supplying NMR spectrometer. 
References

1. S. Goel, B. Jhurani, B. Aggarwal, Mol. Nutr. Food Res. 2008; 52, 1010.

2. R.A. Sharma, A.J. Gescher, W.P. Steward, Eur. J. Cancer 2005; 4, 1955.

3. R.K. Maheshwari, A.K. Singh, J. Gaddipati, R.C. Srimal, Life Sci. 2006; 78, 2081.

4. $\quad$ L. Shen, H.F. Ji, Spectrochim. Acta A Mol. Biomol. Spectrosc. 2007; 67,619.

5. Y. Jiao, J. Wilkinson IV, X. Di, W. Wang, H. Hatcher, N.D. Kock, R. D’Agostino Jr, M.A. Knovich, F.M. Torti, S.V. Torti, Blood 2009; 113462.

6. M. Borsari, E. Ferrari, R. Grandi, M. Saladini, Inorg. Chim. Acta 2002; 328, 61.

7. P. Cornago, R.M. Claramount, L. Bouissane, I. Alkorta, J. Elguero, Tetrahedron 2008; 8089.

8. V. Bertolasi, V. Ferretti, P. Gilli, X. Yao, C. J. Li, New J. Cem. 2008; 32, 694.

9. B. Arezzini, M. Ferrali, E. Ferrari, R. Grandi, S. Monti, M. Saladini, Eur. J. Inorg. Chem. 2004; 3, 646.

10. E. Ferrari, B. Arezzini, M. Ferrali, S. Lazzari, F. Pignedoli, F. Spagnolo, M. Saladini, BioMetals 2009; 22, 701.

11. E. Ferrari, S. Lazzari, G. Marverti, F. Pignedoli, F. Spagnolo, M. Saladini, Biorg. Med. Chem. 2009; 17, 3043.

12. S.M. Khopde, K.I. Priyadarsini, D.K. Palit, T. Mukherjee, Photochem. Photobiol. 2000; 72 , 625.

13. B. Miehlich, A. Savin, H. Stoll, H. Preuss, Chem. Phys. Lett. 1989; 157, 200.

14. W. Lee, R. Yang, G. Parr, Phys. Rev. 1988; B 37, 785.

15. A.D. Becke, J. Chem. Phys. 1993; 98, 5648.

16. M.J. Frisch, G.W. Trucks, H.B. Schlegel, G.E. Scuseria, M.A. Robb, J.R. Cheeseman, J.A. Montgomery Jr., T. Vreven, K.N. Kudin, J.C. Burant, J.M. Millam, S.S. Iyengar, J. Tomasi, V. Barone, B. Mennucci, M. Cossi, G. Scalmani, N. Rega, G.A. Petersson, H. Nakatsuji, M. Hada, M. Ehara, K. Toyota, R. Fukuda, J. Hasegawa, M. Ishida, T. Nakajima, Y. Honda, O. Kitao, H. Nakai, 
M. Klene, X. Li, J.E. Knox, H.P. Hratchian, J.B. Cross, V. Bakken, C. Adamo, J. Jaramillo, R. Gomperts, R.E. Stratmann, O. Yazyev, A.J. Austin, R. Cammi, C. Pomelli, J.W. Ochterski, P.Y. Ayala, K. Morokuma, G.A. Voth, P. Salvador, J.J. Dannenberg, V.G. Zakrzewski, S. Dapprich, A.D. Daniels, M.C. Strain, O. Farkas, D.K. Malick, A.D. Rabuck, K. Raghavachari, J.B. Foresman, J.V. Ortiz, Q. Cui, A.G. Baboul, S. Cliord, J. Cioslowski, B.B. Stefanov, G. Liu, A. Liashenko, P. Piskorz, I. Komaromi, R.L. Martin, D.J. Fox, T. Keith, M.A. Al-Laham, C.Y. Peng, A. Nanayakkara, M. Challacombe, P.M.W. Gill, B. Johnson, W. Chen, M.W. Wong, C. Gonzalez, J.A. Pople, Gaussian 03, Revision C.02, Gaussian, Inc., Wallingford, CT, 2004.

17. R. Benassi, E. Ferrari, S. Lazzari, F. Spagnolo, M Saladini, J. Mol. Struct. 2008; 892, 168.

18. R. Dennington II, T. Keyth, J. Millam, K. Eppinnett, W.L. Hovell, R. Gilliand, Gaussview, Version 3.0, Semichem, Inc.,Shawnee Mission, KS, 2003.

19. S. Miertus, E. Scrocco, J. Tomasi, Chem. Phys. 1981; 55, 117.

20. S. Miertus, J. Tomasi, Chem. Phys. 1982; 65, 239.

21. M. Cossi, V. Barone, Cammi, J. Chem. Phys. Lett. 1996; 255, 327.

22. K. Wolinski, J.F. Hinton, P.J. Pulay, J. Am. Chem. Soc. 1990; 112,8251.

23. K. Nagayama, A. Kumar, K. Wuethrich, R.R. Ernst, J. Magn. Res. 1980; 40, 321.

24. A. Bax, M.F. Summers, J. Am. Chem. Soc. 1986; 108, 2093.

25. A. Bax, R. H.Griffey, B. L. Hawkins, J. Magn. Res. 1983; 55, 301.

26. E. Benassi, F. Spagnolo, Theor. Chem. Acc. 2009; 124, 235.

27. R. Benassi, E. Ferrari, R. Grandi, S. Lazzari, M. Saladini, J. Inorg. Biochem. 2007; 101, 203.

28. Blanco, I. Alkorta, J. Elguero, Magn.Res.Chem 2007; 45, 797.

29. F. M. Szafran, E. Bartoszak-Adamska, J. Koput, Z. Dega-Szafran, J. Mol. Struct. 2007; 140, 844.

30. J.B. Foresman, A. Frisch, "Exploring Chemistry with Electronic Structure Methods: A Guide to Using Gaussian"(2 ed.), Gaussian, Inc, Pittsburgh, PA 1996. 
Table 1- $\theta$ and $\varphi$ dihedral angles, total electronic energies $(E)$, zero point vibrational energies $(E+$ ZPE), thermodynamic quantities $(\mathrm{G}, \mathrm{H})$ and relative differences calculated at B3LYP/6-31G*.

a) Not rotated dihedral angle; b) rotated dihedral angle; c) a.u. ; d) $\mathrm{kcal} \mathrm{mol}^{-1}$

\begin{tabular}{|c|c|c|c|c|c|c|c|c|c|c|c|}
\hline & State & $\theta^{a}$ & $\phi^{\mathbf{b}}$ & $\mathbf{E}^{\mathbf{c}}$ & $\mathbf{E}+\mathbf{Z P E}^{\mathrm{c}}$ & $\mathbf{H}^{\mathbf{c}}$ & $\mathbf{G}^{\mathbf{c}}$ & $\Delta \mathbf{E}^{\mathrm{d}}$ & $\Delta E+Z P E^{d}$ & $\Delta \mathbf{H}^{\mathrm{d}}$ & $\Delta \mathbf{G}^{\mathrm{d}}$ \\
\hline \multirow[t]{4}{*}{ A1 } & $\mathbf{G S}_{1}$ & - & 99.89 & -1795.689556 & -1795.202059 & -1795.166855 & -1795.272771 & 0.00 & 0.00 & 0.00 & 0.00 \\
\hline & $\mathbf{T S}_{1}$ & - & 203.03 & -1795.675170 & -1795.188357 & -1795.153763 & -1795.257743 & 9.03 & 8.60 & 8.22 & 9.43 \\
\hline & $\mathbf{G S}_{2}$ & - & 282.23 & -1795.685569 & -1795.198340 & -1795.162987 & -1795.269849 & 2.50 & 2.33 & 2.43 & 1.83 \\
\hline & $\mathbf{T S}_{2}$ & - & 358.46 & -1795.675225 & -1795.188406 & -1795.153778 & -1795.257568 & 8.99 & 8.57 & 8.21 & 9.54 \\
\hline \multirow[t]{4}{*}{ A2 } & $\mathbf{G S}_{1}$ & - & 71.35 & -1874.295775 & -1873.750883 & -1873.712800 & -1873.826631 & 0.00 & 0.00 & 0.00 & 0.00 \\
\hline & $\mathbf{T S}_{1}$ & - & 216.94 & -1874.286710 & -1873.742105 & -1873.704899 & -1873.814405 & 5.69 & 5.51 & 4.96 & 7.67 \\
\hline & $\mathbf{G S}_{2}$ & - & 293.57 & -1874.293078 & -1873.747762 & -1873.709895 & -1873.822106 & 1.69 & 1.96 & 1.82 & 2.84 \\
\hline & $\mathbf{T S}_{2}$ & - & 347.61 & -1874.288686 & -1873.744054 & -1873.706854 & -1873.816857 & 4.45 & 4.29 & 3.73 & 6.13 \\
\hline \multirow[t]{4}{*}{ A3 } & $\mathbf{G S}_{1}$ & - & 117.93 & -1645.248964 & -1644.770062 & -1644.737057 & -1644.839474 & 0.00 & 0.00 & 0.00 & 0.00 \\
\hline & $\mathbf{T S}_{1}$ & - & 215.56 & -1645.238652 & -1644.760269 & -1644.728023 & -1644.827595 & 6.47 & 6.15 & 5.67 & 7.45 \\
\hline & $\mathbf{G S}_{2}$ & - & 285.75 & -1645.247768 & -1644.768673 & -1644.735734 & -1644.838272 & 0.75 & 0.87 & 0.83 & 0.75 \\
\hline & $\mathbf{T S}_{2}$ & - & 354.04 & -1645.240588 & -1644.762127 & -1644.729889 & -1644.829315 & 5.26 & 4.98 & 4.50 & 6.37 \\
\hline \multirow[t]{4}{*}{ C1 } & $\mathbf{G S}_{1}$ & -100.42 & 100.33 & -2406.423034 & -2405.761907 & -2405.715397 & -2405.848672 & 0.00 & 0.00 & 0.00 & 0.00 \\
\hline & $\mathbf{T S}_{1}$ & -100.76 & 203.85 & -2406.408721 & -2405.748111 & -2405.702281 & -2405.832897 & 8.98 & 8.66 & 8.23 & 9.90 \\
\hline & $\mathbf{G S}_{2}$ & -100.38 & 283.45 & -2406.419166 & -2405.757980 & -2405.711444 & -2405.843949 & 2.43 & 2.46 & 2.48 & 2.96 \\
\hline & $\mathbf{T S}_{2}$ & -101.45 & 358.72 & -2406.408670 & -2405.748086 & -2405.702199 & -2405.832666 & 9.01 & 8.67 & 8.28 & 10.04 \\
\hline \multirow[t]{4}{*}{$\mathrm{C2}$} & $\mathbf{G S}_{1}$ & -70.29 & 70.21 & -2485.008984 & -2484.291502 & -2484.241500 & -2484.383139 & 0.00 & 0.00 & 0.00 & 0.00 \\
\hline & $\mathbf{T S}_{1}$ & -69.85 & 216.44 & -2484.999813 & -2484.282656 & -2484.233480 & -2484.372251 & 5.75 & 5.55 & 5.03 & 6.83 \\
\hline & $\mathbf{G S}_{2}$ & -70.32 & 292.82 & -2485.005972 & -2484.288132 & -2484.238297 & -2484.379928 & 1.89 & 2.11 & 2.01 & 2.01 \\
\hline & $\mathbf{T S}_{2}$ & -70.41 & 347.01 & -2485.001852 & -2484.284795 & -2484.235586 & -2484.374735 & 4.47 & 4.21 & 3.71 & 5.27 \\
\hline \multirow[t]{4}{*}{$\mathrm{C3}$} & $\mathbf{G S}_{1}$ & -121.28 & 121.21 & -2255.973714 & -2255.321410 & -2255.276831 & -2255.406096 & 0.00 & 0.00 & 0.00 & 0.00 \\
\hline & $\mathbf{T S}_{1}$ & -121.24 & 214.87 & -2255.963342 & -2255.311860 & -2255.267906 & -2255.396296 & 6.50 & 5.99 & 5.69 & 6.15 \\
\hline & $\mathbf{G S}_{2}$ & -119.54 & 289.28 & -2255.972864 & -2255.320652 & -2255.276018 & -2255.404949 & 0.53 & 0.48 & 0.52 & 0.72 \\
\hline & $\mathbf{T S}_{2}$ & -121.64 & 353.48 & -2255.965306 & -2255.313594 & -2255.269723 & -2255.396658 & 5.27 & 4.90 & 4.53 & 5.92 \\
\hline
\end{tabular}


Table 2- CHELPG charges calculated with B3LYP/6-31G* basis-set

\begin{tabular}{|c|c|c|c|c|}
\hline \multirow{2}{*}{ Molecule } & \multicolumn{4}{|c|}{ CHELPG Charges } \\
\hline & $=0$ & $-\mathrm{OH}$ & $\mathbf{0 3 2}$ & $\mathbf{O 3 3}$ \\
\hline Curcumin & $-0.595 *$ & $-0.579 *$ & & \\
\hline A1 & -0.584 & -0.572 & -0.553 & -0.544 \\
\hline A2 & -0.594 & -0.576 & & \\
\hline A3 & -0.597 & -0.569 & & \\
\hline \multirow[t]{5}{*}{ C1 } & -0.591 & -0.571 & -0.532 & -0.534 \\
\hline & $-0.646(\mathrm{w})$ & $-0.600(w)$ & $-0.572(\mathrm{w})$ & $-0.573(\mathrm{w})$ \\
\hline & $-0.591(\mathrm{~m})$ & $-0.571(\mathrm{~m})$ & $-0.533(\mathrm{~m})$ & $-0.534(\mathrm{~m})$ \\
\hline & $-0.623(a)$ & $-0.587(\mathrm{a})$ & $-0.554(a)$ & $-0.556(a)$ \\
\hline & $-0.623(\mathrm{~d})$ & $-0.587(\mathrm{~d})$ & $-0.555(\mathrm{~d})$ & $-0.556(\mathrm{~d})$ \\
\hline \multirow[t]{5}{*}{$\mathrm{C} 2$} & -0.600 & -0.580 & & \\
\hline & $-0.641(w)$ & $-0.595(\mathrm{w})$ & & \\
\hline & $-0.639(\mathrm{~m})$ & $-0.594(\mathrm{~m})$ & & \\
\hline & $-0.620(a)$ & $-0.584(a)$ & & \\
\hline & $-0.620(d)$ & $-0.584(\mathrm{~d})$ & & \\
\hline \multirow{5}{*}{$\mathrm{C3}$} & -0.601 & -0.582 & & \\
\hline & $-0.654(w)$ & $-0.608(w)$ & & \\
\hline & $-0.601(\mathrm{~m})$ & $-0.582(\mathrm{~m})$ & & \\
\hline & $-0.632(a)$ & $-0.596(a)$ & & \\
\hline & $-0.632(d)$ & $-0.598(d)$ & & \\
\hline
\end{tabular}

*Values taken from Ref 15 . Values calculated in vacuum if not specified, (w) water, (m) MeOH, (a) ACN, (d) DMSO. 
Table 3- Calculated averaged GIAO magnetic isotropic shielding tensors $\left(\bar{\sigma}_{\text {calc }}\right)$ for $\mathbf{C 1}, \mathbf{C 2}$ and $\mathbf{C 3}$ in vacuum (v), MeOH (m) and DMSO (d).

\begin{tabular}{|c|c|c|c|c|c|c|c|c|c|}
\hline \multirow[b]{2}{*}{${ }^{13} \mathrm{C}$} & \multicolumn{3}{|c|}{ C1 } & \multicolumn{3}{|c|}{$\mathrm{C2}$} & \multicolumn{3}{|c|}{$\mathrm{C3}$} \\
\hline & $\mathrm{V}$ & $\overline{\mathrm{m}}$ & $\mathrm{d}$ & $\mathrm{V}$ & $\mathrm{m}$ & $\mathrm{d}$ & $\mathrm{v}$ & $\mathrm{m}$ & $\mathrm{d}$ \\
\hline 1 & 88.34 & 87.94 & 88.01 & 88.5 & 88.19 & 88.29 & 88.75 & 88.64 & 88.62 \\
\hline 2 & 17.99 & 17.36 & 17.65 & 18.13 & 17.46 & 17.77 & 18.14 & 17.54 & 17.82 \\
\hline 3 & 72.92 & 72.8 & 72.91 & 73.34 & 73.18 & 73.37 & 74.28 & 74.47 & 74.47 \\
\hline 4 & 54.21 & 53.84 & 54.01 & 54.69 & 54 & 54.2 & 55.27 & 54.56 & 54.82 \\
\hline 5 & 62.48 & 63.47 & 63.29 & 64.33 & 64.36 & 64.45 & 66.69 & 67.35 & 67.23 \\
\hline 6 & 73.19 & 73.98 & 73.96 & 79.16 & 77.99 & 78.37 & 60.88 & 60.51 & 60.69 \\
\hline 7 & 45.32 & 46.03 & 45.79 & 44.92 & 44.85 & 44.83 & 76.35 & 76.59 & 76.57 \\
\hline 8 & 49.42 & 48.2 & 48.58 & 48.22 & 48.5 & 48.41 & 36.48 & 35.8 & 36.03 \\
\hline 9 & 71.79 & 71.02 & 70.95 & 68.91 & 69.3 & 69.1 & 78.25 & 77.66 & 77.72 \\
\hline 10 & 81.35 & 79.7 & 80.11 & 78.51 & 77.95 & 78.11 & 70.32 & 69.18 & 69.41 \\
\hline 11 & 84.48 & 85.92 & 85.26 & 89.05 & 89.5 & 89.35 & 86.84 & 87.8 & 87.33 \\
\hline 12 & 115.66 & 116.23 & 115.72 & 115.9 & 116.54 & 115.88 & 116.26 & 116.88 & 116.26 \\
\hline 13 & 114.94 & 115.9 & 115.4 & 113.79 & 114.9 & 114.23 & 114.3 & 115.37 & 114.81 \\
\hline 14 & 119.61 & 120.01 & 119.61 & 119.83 & 120.26 & 119.81 & 120.2 & 120.63 & 120.2 \\
\hline 15 & 114.05 & 115.18 & 114.52 & 114.92 & 116.03 & 115.35 & 113.73 & 114.89 & 114.26 \\
\hline 16 & 126.04 & 126.7 & 126.47 & 126.08 & 126.87 & 126.56 & 125.87 & 126.58 & 126.31 \\
\hline${ }^{1} \mathrm{H}$ & & & & & & & & & \\
\hline 1 & 27.27 & 26.76 & 26.96 & 27.22 & 26.74 & 26.94 & 27.28 & 26.79 & 26.99 \\
\hline 3 & 26.05 & 25.64 & 25.82 & 26.02 & 25.63 & 25.82 & 26.08 & 25.7 & 25.86 \\
\hline 4 & 24.85 & 24.85 & 24.9 & 24.87 & 24.8 & 24.86 & 24.85 & 24.83 & 24.89 \\
\hline 6 & 25.57 & 25.42 & 25.57 & 25.89 & 25.52 & 25.7 & 25.1 & 24.89 & 25.01 \\
\hline 7 & I & I & I & 1 & I & 1 & 25.16 & 25.06 & 25.13 \\
\hline 9 & 25.19 & 24.91 & 25.03 & 25.16 & 25.01 & 25.12 & 25.34 & 25.06 & 25.18 \\
\hline 10 & 25.22 & 24.89 & 25.01 & 24.97 & 24.72 & 24.83 & 24.61 & 24.32 & 24.43 \\
\hline 11 & 27.82 & 26.98 & 27.53 & 26.89 & 26.73 & 26.86 & 27.36 & 26.69 & 27.15 \\
\hline 12 & 28.49 & 28.23 & 28.47 & 28.43 & 28.21 & 28.47 & 28.39 & 28.18 & 28.42 \\
\hline 13 & 28.6 & 28.19 & 28.44 & 28.7 & 28.28 & 28.57 & 28.6 & 28.17 & 28.43 \\
\hline 14 & 28.2 & 28.03 & 28.22 & 28.21 & 28.04 & 28.25 & 28.2 & 28.04 & 28.24 \\
\hline 15 & 28.94 & 28.37 & 28.69 & 29.09 & 28.53 & 28.88 & 28.87 & 28.27 & 28.6 \\
\hline 16 & 28 & 27.86 & 27.9 & 28.16 & 28 & 28.06 & 28.01 & 27.85 & 27.9 \\
\hline 16 & 28.14 & 28.12 & 28.14 & 28.41 & 28.39 & 28.42 & 28.22 & 28.2 & 28.23 \\
\hline
\end{tabular}


Table 4-Experimental $\left(\delta_{\text {exp }}\right)$ and predicted $\left(\delta_{\text {pred }}=\mathrm{a} \cdot \bar{\sigma}_{\text {calc }}+\mathrm{b}\right){ }^{13} \mathrm{C}$ chemical shifts $(\mathrm{ppm})$ for $\mathbf{C 1}, \mathbf{C 2}$ and $\mathbf{C 3}$ in vacuum $(\mathrm{v}), \mathrm{MeOH}(\mathrm{m})$ and $\operatorname{DMSO}(\mathrm{d})$.

\begin{tabular}{|c|c|c|c|c|c|c|c|c|c|c|c|c|c|c|c|c|c|c|}
\hline & \multicolumn{6}{|c|}{ C1 } & \multicolumn{6}{|c|}{$\mathrm{C2}$} & \multicolumn{6}{|c|}{$\mathrm{C3}$} \\
\hline & $\begin{array}{l}\delta_{\exp } \\
(\mathrm{m})\end{array}$ & $\begin{array}{r}\text { opred } \\
(\mathrm{V})\end{array}$ & $\begin{array}{r}\delta_{\text {pred }} \\
(\mathrm{m}) \\
\end{array}$ & $\begin{array}{r}\delta_{\exp } \\
(\mathrm{d}) \\
\end{array}$ & $\begin{array}{r}\delta_{\text {pred }} \\
(\mathrm{v}) \\
\end{array}$ & $\begin{array}{r}\delta_{\text {pred }} \\
\text { (d) }\end{array}$ & $\begin{array}{l}\delta_{\exp } \\
(\mathrm{m}) \\
\end{array}$ & $\begin{array}{r}\delta_{\text {pred }} \\
(\mathrm{v})\end{array}$ & $\begin{array}{r}\delta_{\text {pred }} \\
(\mathrm{m}) \\
\end{array}$ & $\begin{array}{r}\delta_{\exp } \\
\text { (d) } \\
\end{array}$ & $\begin{array}{r}\delta_{\text {pred }} \\
(\mathrm{v}) \\
\end{array}$ & $\begin{array}{r}\delta_{\text {pred }} \\
\text { (d) }\end{array}$ & $\begin{array}{l}\delta_{\text {exp }} \\
(\mathrm{m}) \\
\end{array}$ & (v) & $\begin{array}{r}\delta_{\text {pred }} \\
(\mathrm{m})\end{array}$ & $\begin{array}{r}\delta_{\exp } \\
\text { (d) }\end{array}$ & $\begin{array}{r}\delta_{\text {pred }} \\
(\mathrm{d}) \\
\end{array}$ & $\begin{array}{l}\delta_{\text {exp }} \\
(\mathrm{m}) \\
\end{array}$ \\
\hline 1 & 2.80 & 8185 & 22.50 & 1.71 & 93.19 & 3.62 & 2.80 & 02.08 & 02.56 & 1.32 & 03.50 & 03.76 & $\overline{02.8}$ & 102.4 & 102.8 & 1.74 & 03.82 & 4.01 \\
\hline 2 & .40 & 4.41 & 4.20 & & 80.20 & & .60 & & & & & & & & 86.1 & & .87 & 1.69 \\
\hline 3 & 4.50 & 119.95 & 120.04 & 2.77 & 120.07 & 120.07 & 4.50 & 19.92 & 9.89 & & 0.11 & 0.01 & 4.5 & 119.7 & 119.5 & .81 & 9.81 & 9.54 \\
\hline 4 & 2.10 & 141.90 & 141.98 & 40.56 & 140.55 & 140.66 & 2.10 & 41.86 & 42.12 & 0.69 & 140.53 & 140.89 & 142.1 & 142.4 & 142.6 & 0.31 & 140.82 & 41.10 \\
\hline 5 & 2.60 & 132.20 & 31.22 & 29.54 & 131.50 & 55 & 2.10 & 130.52 & 30.25 & 8.91 & 129.97 & 129.73 & 131.6 & 128.8 & 128.0 & 3.78 & 128.20 & 127.48 \\
\hline 6 & 620 & 119.63 & 18.83 & & 119.78 & 11 & 12.10 & 113.07 & 114.07 & 60 & 73 & 57 & 131.6 & 135.7 & 135.7 & & & 4.66 \\
\hline 7 & .70 & 4 & 3 & & 150 & & 9.60 & .36 & 99 & & & & 18.9 & 117.2 & 17.0 & & .52 & 7.24 \\
\hline 8 & .40 & 53 & & & & & 0 & & & & & & & & 4.7 & & 60 & .71 \\
\hline 9 & & & & & & & & & & & & & & & 5.7 & & & .97 \\
\hline 10 & & & & & & & & & & & & & & & 5.4 & & 19 & 5.09 \\
\hline 11 & 96.30 & 106.38 & 105.70 & .58 & 107.42 & 61 & 40 & 101.43 & 32 & & 90 & 61 & 96.5 & 104.7 & 104.4 & .34 & .93 & 105.43 \\
\hline 12 & 10 & 69.79 & 70.34 & 76.96 & 73.28 & & 10 & 69.84 & 70.52 & & 3.50 & .71 & 8.4 & 69.5 & 70.3 & .55 & .41 & 3.69 \\
\hline 13 & 71.40 & 70.64 & 70.73 & .92 & 74.07 & 73.78 & 70.50 & 72.32 & 72.44 & 77.23 & 75.81 & 75.51 & 71.2 & 71.9 & 72.1 & .98 & .57 & 75.28 \\
\hline 14 & 65.00 & 65.16 & 65.81 & 73.42 & 68.96 & 69.19 & 66.20 & 65.22 & 65.95 & 69.97 & 69.19 & 69.43 & 66.7 & 64.8 & 65.7 & 70.06 & 69.05 & 69.37 \\
\hline 15 & 70.10 & 71.68 & 71.74 & & 75.05 & & 69.70 & 71.00 & 71.14 & & 74.57 & 74.29 & 68.7 & 72.6 & 72.7 & 52 & 76.20 & 75.89 \\
\hline 16 & 59.20 & 57.61 & 57.86 & 60.80 & 61.92 & 61.72 & 59.20 & 57.86 & 58.12 & 61.14 & 62.35 & 62.08 & 58.3 & 58.0 & 58.5 & 61.07 & 62.78 & 62.67 \\
\hline $\mathrm{a}$ & & -1.174 & -1.161 & & -1.095 & -1.089 & & 1.177 & -1.161 & & 1.095 & & & .195 & 176 & & 1.105 & 1.097 \\
\hline $\mathrm{b}$ & & 205.52 & 204.69 & & 199.90 & 199.50 & & 206.21 & 205.06 & & 200.42 & 19 & & 8.51 & 207.09 & & & 1.24 \\
\hline $\mathrm{R}^{2}$ & & 0.9834 & 0.9878 & & 0.9905 & 0.9915 & & 0.9906 & 0.9917 & & 0.9879 & 0.9882 & & 0.9896 & 0.9911 & & 0.9933 & 0.9939 \\
\hline
\end{tabular}


Table 5- Experimental $\left(\delta_{\text {exp }}\right)$ and predicted $\left(\delta_{\text {pred }}=\mathrm{a} \cdot \bar{\sigma}_{\text {calc }}+\mathrm{b}\right){ }^{1} \mathrm{H}$ chemical shifts (ppm) for C1, C2 and C3 in vacuum (v). MeOH (m) and DMSO

(d).

\begin{tabular}{|c|c|c|c|c|c|c|c|c|c|c|c|c|c|c|c|c|c|c|}
\hline \multirow[b]{2}{*}{${ }^{1} \mathrm{H}$} & \multicolumn{6}{|c|}{$\mathrm{C1}$} & \multicolumn{6}{|c|}{$\mathrm{C2}$} & \multicolumn{6}{|c|}{ C3 } \\
\hline & $\begin{array}{l}\delta_{\exp } \\
(\mathrm{m})\end{array}$ & $\begin{array}{c}\delta_{\text {pred }} \\
(\mathrm{v})\end{array}$ & $\begin{array}{c}\delta_{\text {pred }} \\
(\mathrm{m})\end{array}$ & $\begin{array}{c}\delta_{\exp } \\
(\mathrm{d})\end{array}$ & $\begin{array}{c}\delta_{\text {pred }} \\
\text { (v) }\end{array}$ & $\begin{array}{c}\delta_{\text {pred }} \\
\text { (d) }\end{array}$ & $\begin{array}{l}\delta_{\exp } \\
(\mathrm{m})\end{array}$ & $\begin{array}{c}\delta_{\text {pred }} \\
\text { (v) }\end{array}$ & $\begin{array}{c}\delta_{\text {pred }} \\
(\mathrm{m})\end{array}$ & $\begin{array}{l}\delta_{\exp } \\
(\mathrm{d})\end{array}$ & $\begin{array}{c}\delta_{\text {pred }} \\
\text { (v) }\end{array}$ & $\begin{array}{c}\delta_{\text {pred }} \\
\text { (d) }\end{array}$ & $\begin{array}{l}\delta_{\exp } \\
(\mathrm{m})\end{array}$ & $\begin{array}{c}\delta_{\text {pred }} \\
\text { (v) }\end{array}$ & $\begin{array}{c}\delta_{\text {pred }} \\
(\mathrm{m})\end{array}$ & $\begin{array}{c}\delta_{\exp } \\
\text { (d) }\end{array}$ & $\begin{array}{c}\delta_{\text {pred }} \\
\text { (d) }\end{array}$ & $\begin{array}{c}\delta_{\text {pred }} \\
(\mathrm{m})\end{array}$ \\
\hline 1 & 6.01 & 5.05 & 5.27 & 6.12 & 4.96 & 5.17 & 6.02 & 5.07 & 5.31 & 6.13 & 5.03 & 5.25 & 6.01 & 4.98 & 4.97 & 6.11 & 4.89 & 5.09 \\
\hline 3 & 6.63 & 6.40 & 6.58 & 6.75 & 6.39 & 6.54 & 6.72 & 6.38 & 6.56 & 6.87 & 6.44 & 6.57 & 6.69 & 6.31 & 6.26 & 6.83 & 6.31 & 6.44 \\
\hline 4 & 7.53 & 7.74 & 7.50 & 7.52 & 7.80 & 7.64 & 7.6 & 7.63 & 7.50 & 7.6 & 7.79 & 7.71 & 7.62 & 7.67 & 7.40 & 7.61 & 7.76 & 7.60 \\
\hline 6 & 7.14 & 6.94 & 6.83 & 7.13 & 6.96 & 6.84 & 7.12 & 6.52 & 6.69 & 7.38 & 6.59 & 6.71 & 7.14 & 7.40 & 7.25 & 7.7 & 7.46 & 7.46 \\
\hline 7 & I & I & I & I & I & 1 & I & I & I & I & I & I & 7.61 & 7.33 & 7.11 & 7.09 & 7.39 & 7.32 \\
\hline 9 & 7.20 & 7.36 & 7.43 & 7.13 & 7.40 & 7.48 & 7.18 & 7.31 & 7.26 & 7.13 & 7.45 & 7.40 & 7.60 & 7.13 & 7.05 & 7.09 & 7.18 & 7.26 \\
\hline 10 & 7.07 & 7.32 & 7.45 & 7.2 & 7.37 & 7.51 & 7.18 & 7.52 & 7.59 & 7.26 & 7.67 & 7.74 & 7.14 & 7.94 & 7.92 & 7.7 & 8.04 & 8.15 \\
\hline 11 & 4.97 & 4.44 & 5.01 & 4.81 & 4.31 & 4.49 & 4.98 & 5.43 & 5.32 & 4.99 & 5.42 & 5.34 & 4.98 & 4.90 & 4.79 & 4.96 & 4.79 & 4.90 \\
\hline 12 & 3.53 & 3.69 & 3.55 & 3.30 & 3.52 & 3.37 & 3.53 & 3.75 & 3.65 & 3.27 & 3.61 & 3.44 & 3.52 & 3.76 & 3.33 & 3.27 & 3.58 & 3.38 \\
\hline 13 & 3.47 & 3.57 & 3.60 & 3.18 & 3.40 & 3.40 & 3.48 & 3.45 & 3.57 & 3.28 & 3.29 & 3.32 & 3.47 & 3.52 & 3.30 & 3.29 & 3.33 & 3.37 \\
\hline 14 & 3.44 & 4.02 & 3.79 & 3.32 & 3.87 & 3.67 & 3.43 & 3.99 & 3.84 & 3.17 & 3.87 & 3.70 & 3.42 & 3.97 & 3.55 & 3.17 & 3.80 & 3.60 \\
\hline 15 & 3.45 & 3.19 & 3.39 & 3.36 & 3.00 & 3.10 & 3.45 & 3.03 & 3.28 & 3.34 & 2.83 & 2.95 & 3.45 & 3.22 & 3.11 & 3.37 & 3.01 & 3.17 \\
\hline 16 & 3.90 & 4.24 & 3.98 & 3.72 & 4.10 & 4.05 & 3.89 & 4.04 & 3.88 & 3.67 & 3.93 & 3.92 & 3.91 & 4.18 & 3.93 & 3.69 & 4.03 & 4.00 \\
\hline $16^{\prime}$ & 3.71 & 4.08 & 3.68 & 3.48 & 3.94 & 3.76 & 3.71 & 3.77 & 3.44 & 3.45 & 3.63 & 3.50 & 3.71 & 3.94 & 3.55 & 3.46 & 3.78 & 3.61 \\
\hline $\mathrm{a}$ & & -1.110 & -1.168 & & -1.175 & -1.197 & & -1.091 & -1.131 & & -1.175 & -1.183 & & -1.107 & -1.150 & & -1.181 & -1.196 \\
\hline b & & 35.324 & 36.527 & & 37.003 & 37.442 & & 34.772 & 35.537 & & 37.019 & 37.1198 & & 35.183 & 36.005 & & 37.103 & 37.359 \\
\hline $\mathrm{R}^{2}$ & & 0.9399 & 0.9718 & & 0.9295 & 0.9553 & & 0.9232 & 0.9524 & & 0.9186 & 0.9465 & & 0.9362 & 0.9479 & & 0.9399 & 0.9611 \\
\hline
\end{tabular}


Table 6- Uv-vis band maximum position (nm) in different solvents for compounds $\mathbf{C}$.

\begin{tabular}{|l|c|c|c|c|c|c|c|c|c|}
\hline & Vacuum & \multicolumn{2}{|c|}{$\mathrm{H}_{2} \mathrm{O}$} & \multicolumn{2}{c|}{$\mathrm{MeOH}$} & \multicolumn{2}{c|}{ ACN } & \multicolumn{2}{c|}{ DMSO } \\
\hline & calc & $\exp$ & calc & $\exp$ & calc & exp & calc & exp & calc \\
\hline C1 & 402 & 414 & 423 & 411 & 422 & 405 & 419 & 423 & 421 \\
\hline C2 & 413 & 413 & 432 & 411 & 432 & 413 & 428 & 425 & 431 \\
\hline C3 & 403 & 408 & 425 & 406 & 425 & 405 & 420 & 416 & 423 \\
\hline
\end{tabular}




\section{Caption of Figures}

Figure 1: General structure of the studied curcumin glucosyl derivatives.

Figure 2: $\Delta \mathrm{G}$ for compounds $\mathbf{A 1}, \mathbf{A 2}$ and $\mathbf{A 3}$ as a function of the rotational dihedral angle $\phi$.

Figure 3: Final optimized structures of $\mathbf{A}$ compounds at B3LYP/6-31G* level at $\mathrm{GS}_{1}$ and $\mathrm{GS}_{2}$ states. The hydrogen interaction is represented by dots.

Figure 4: Top view of $\mathbf{C 2}$ optimized structures at B3LYP/6-31G* level showing the different sugar spatial positions with respect to curcumin planar skeleton and their total energies (a.u.).

Figure 5: Representation of HOMO and LUMO orbital density for $\mathbf{A}$ and $\mathbf{C}$ series at B3LYP/6$31 \mathrm{G}^{*}$ level.

Figure 6: Molecular electrostatic maps in vacuum of compounds $\mathbf{A}$ and $\mathbf{C}$ in their $\mathrm{GS}_{1}$ states at B3LYP/6-31G* level.

Figure 7: ${ }^{1} \mathrm{H}$ NMR spectrum of $\mathbf{C 1}$ in DMSO- $d_{6}\left({ }^{*}\right.$ residual DMSO)

Figure 8: Plots of DMSO- $d_{6} v s . \mathrm{MeOD}$ experimental ${ }^{1} \mathrm{H}(\mathbf{A})$ and ${ }^{13} \mathrm{C}(\mathbf{B})$ chemical shifts $(\delta)$ for glucosyl-curcuminoids: C1 ( $\nabla)$. C2 ( $\square)$ and $\mathbf{C 3}(\circ)$.

Figure 9: Plot of ${ }^{1} \mathrm{H}$ and ${ }^{13} \mathrm{C} \delta_{\text {exp }}$ vs. $\bar{\sigma}_{\text {calc }}$ for $\mathbf{C 1}$. Solid circle is referred to $\mathrm{H}-1$ proton; dotted circle highlights anomeric proton (H-11).

Figure 10: Correlation between experimental and B3LYP/6-311G* in vacuum calculated wavenumbers for C1. Equation: $\bar{\nu}_{\text {exp }}=a \cdot \bar{v}_{\text {calc }} ; \mathrm{a}=0.9991, \mathrm{R}^{2}=0.9983$. 
Figure 1

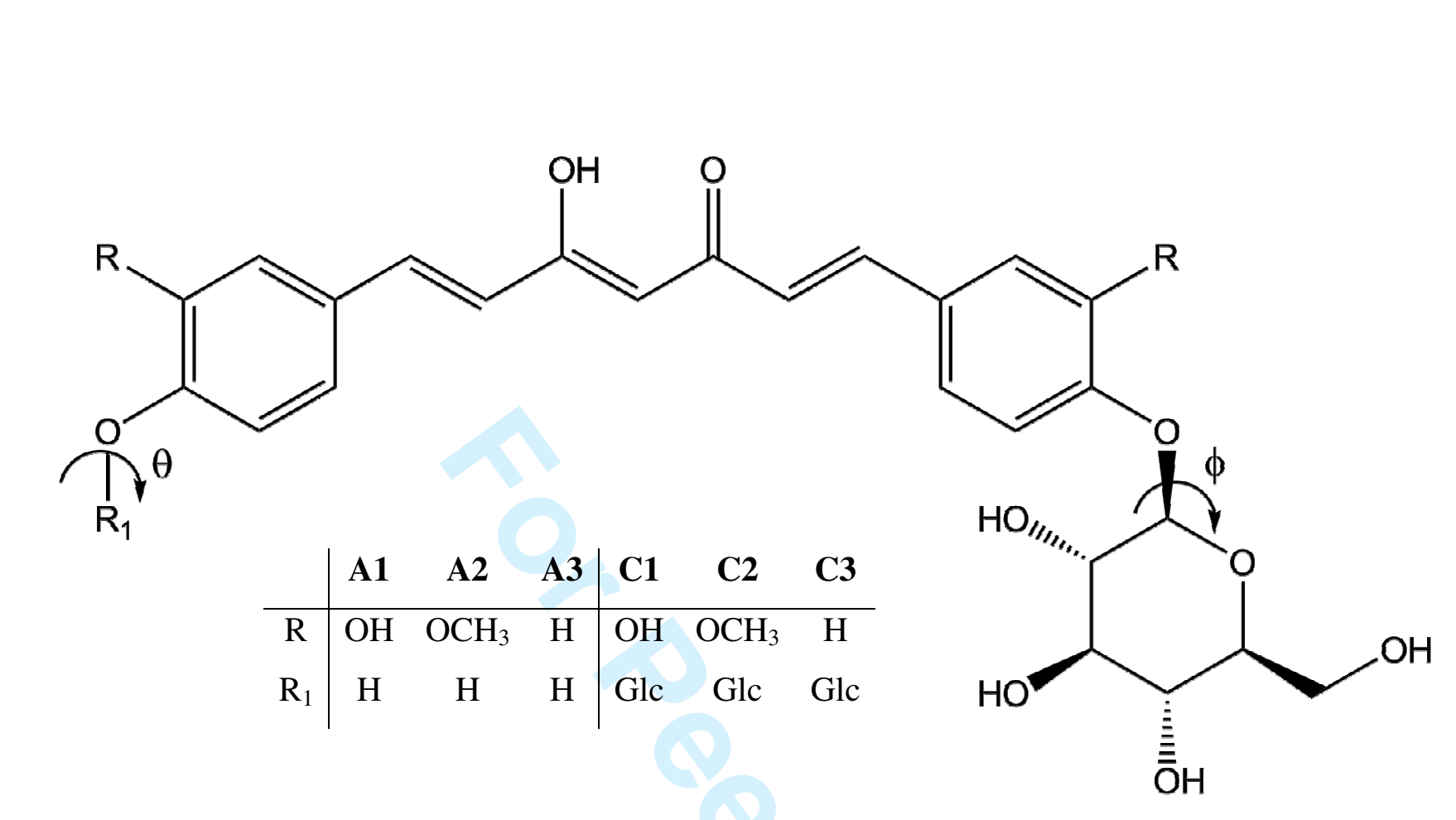


Figure 2

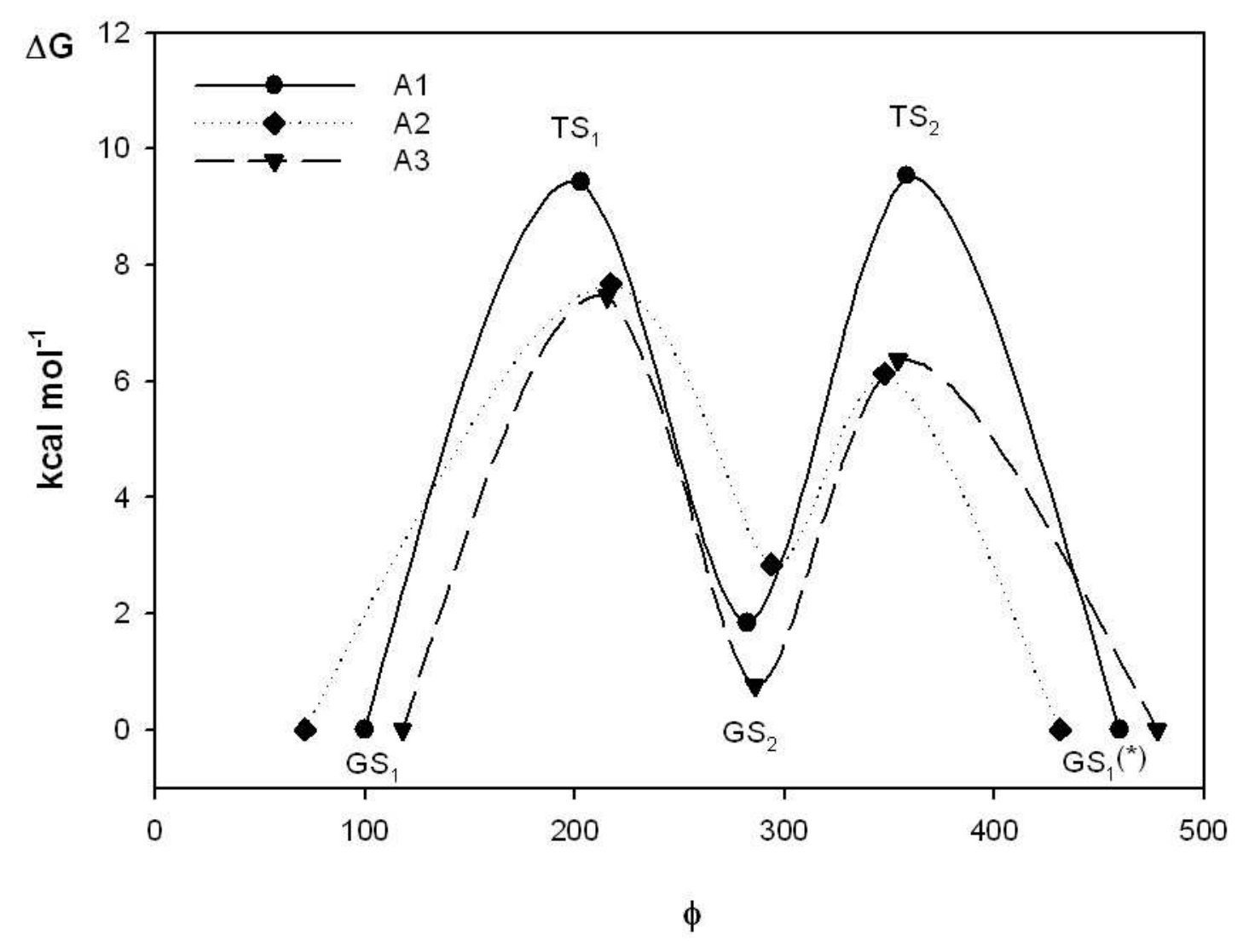




\section{Figure 3}

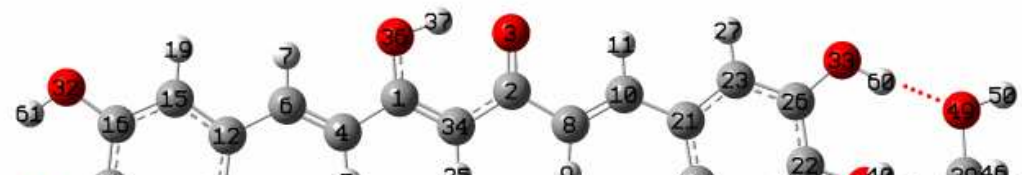

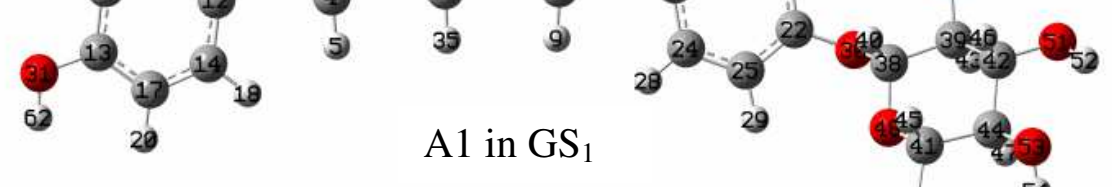

$$
\begin{aligned}
& 5 \text { ริ }
\end{aligned}
$$

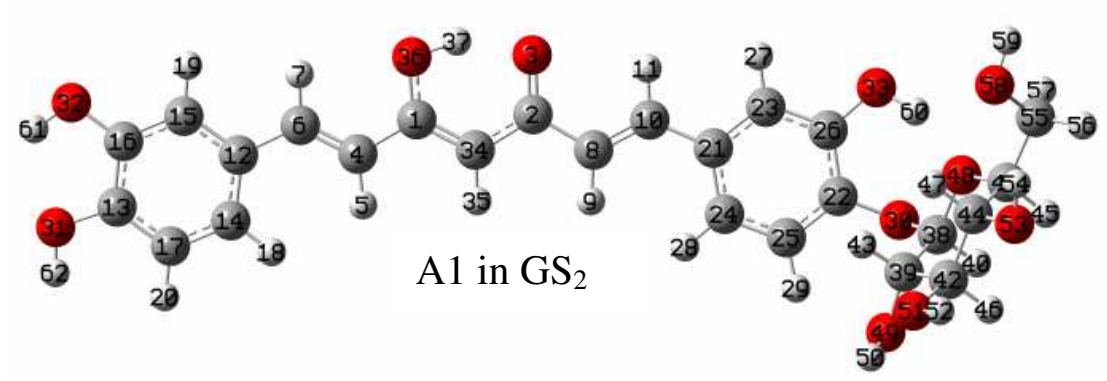

A2 in $\mathrm{GS}_{1}$
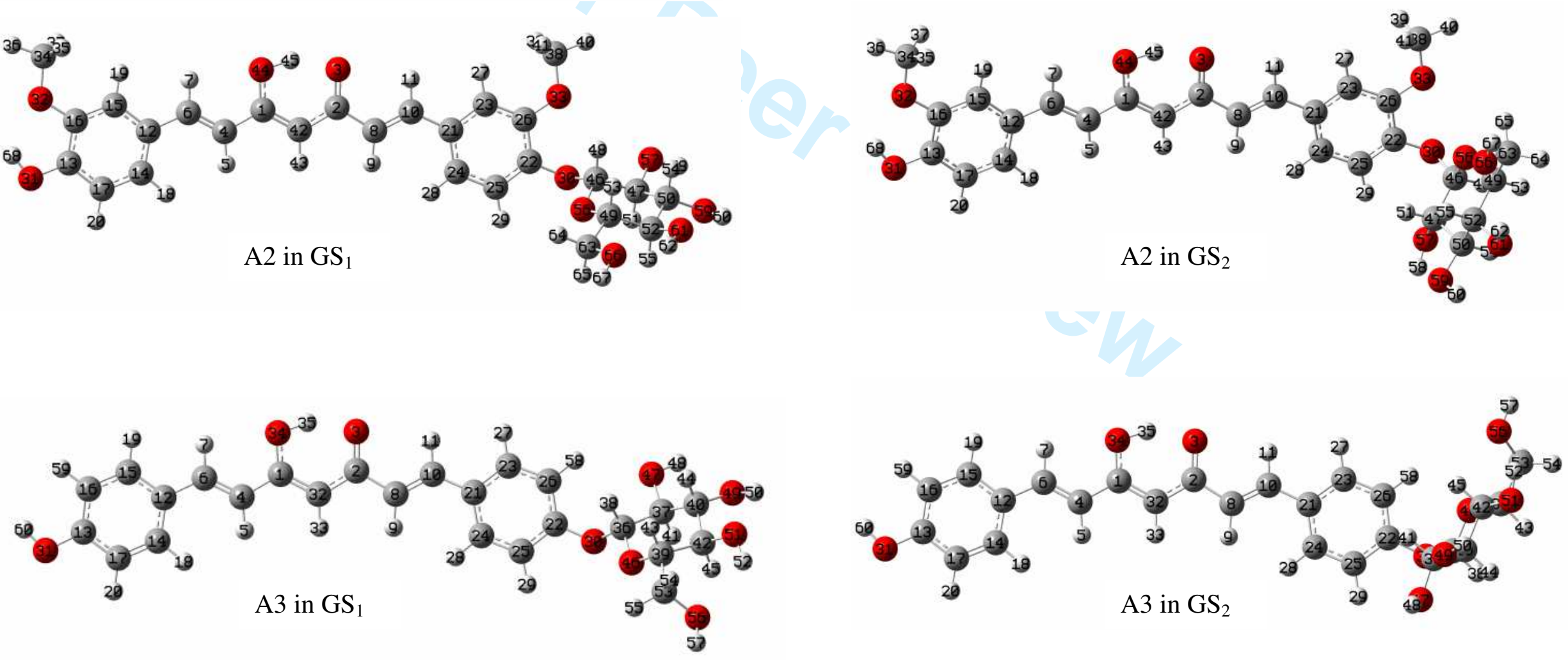
Figure 4

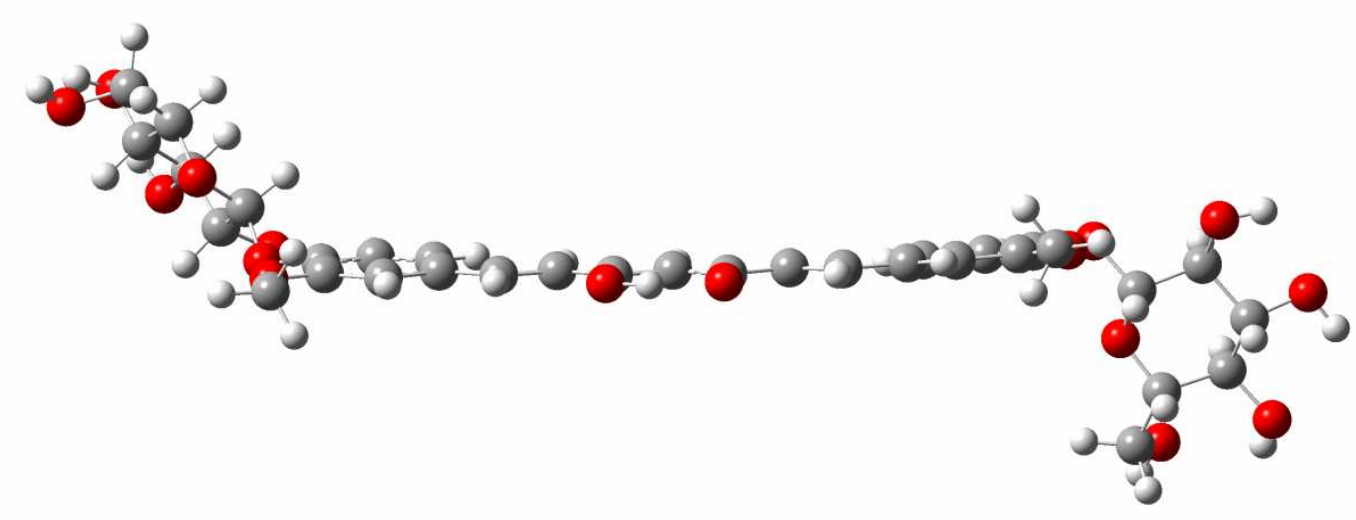

sin-anti configuration $\mathrm{E}=-2485.008088$ a.u.

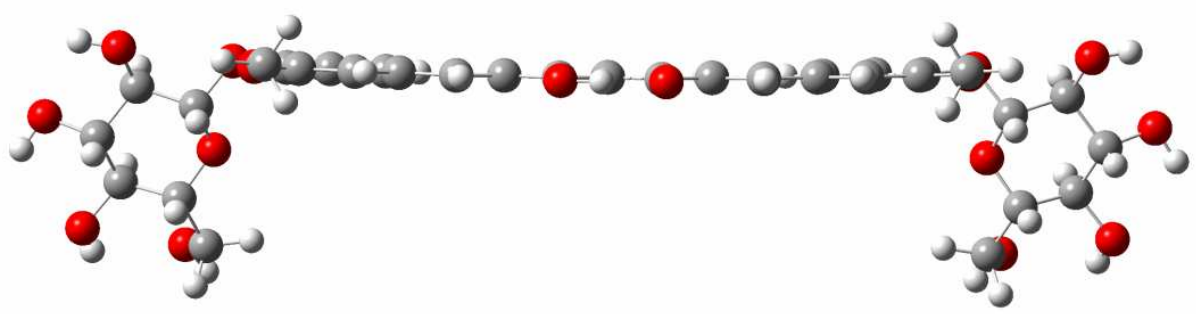

$\sin$ - $\sin$ configuraton $\mathrm{E}=-2485.008984$ a.u. 
Figure 5
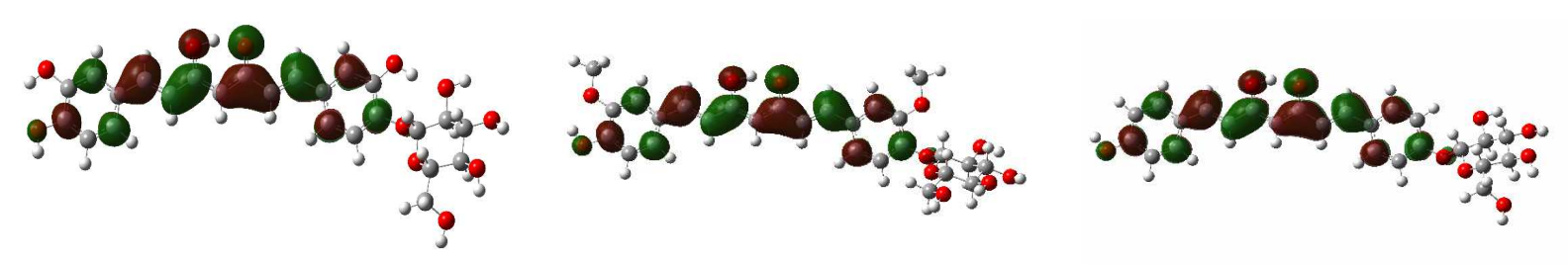
A1 HOMO
A2 HOMO
A3 HOMO

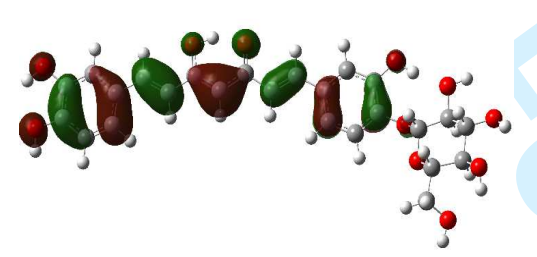

A1 LUMO

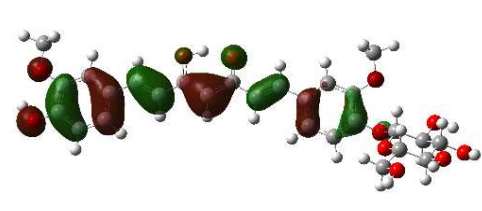

A2 LUMO

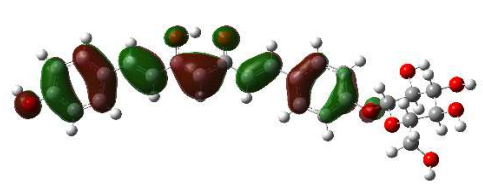

A3 LUMO
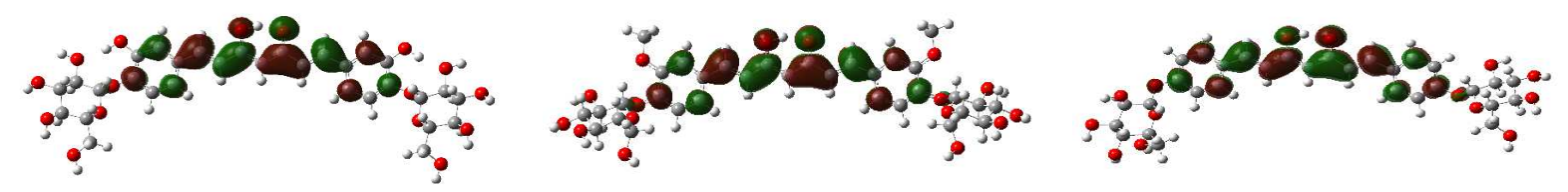

C1 HOMO

C2 HOMO

C3 HOMO

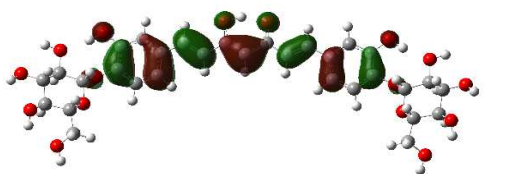

C1 LUMO

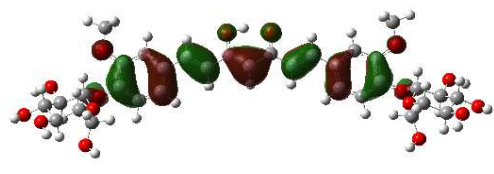

C2 LUMO

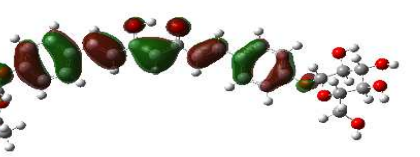

C3 LUMO 
Figure 6

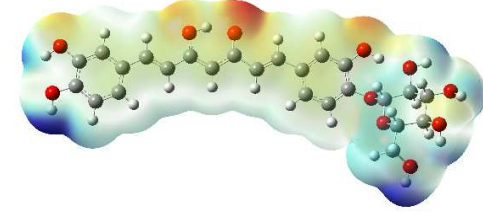

A1

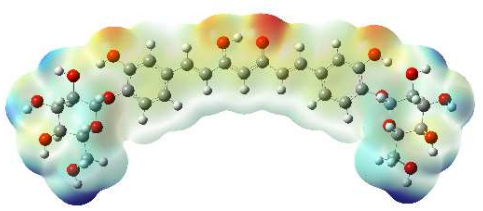

C1

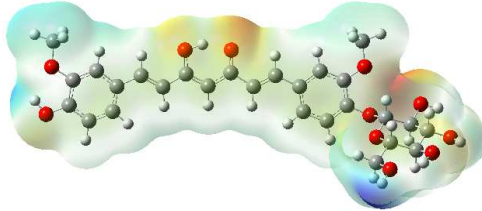

A2

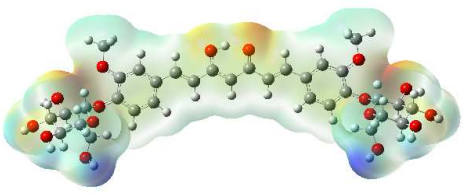

$\mathrm{C} 2$

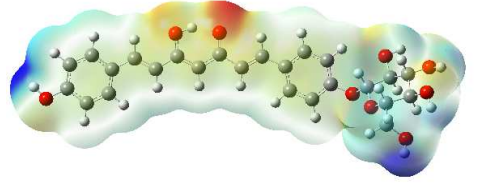

A3

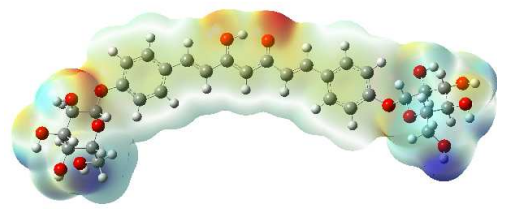

C3 


\section{Figure 7}

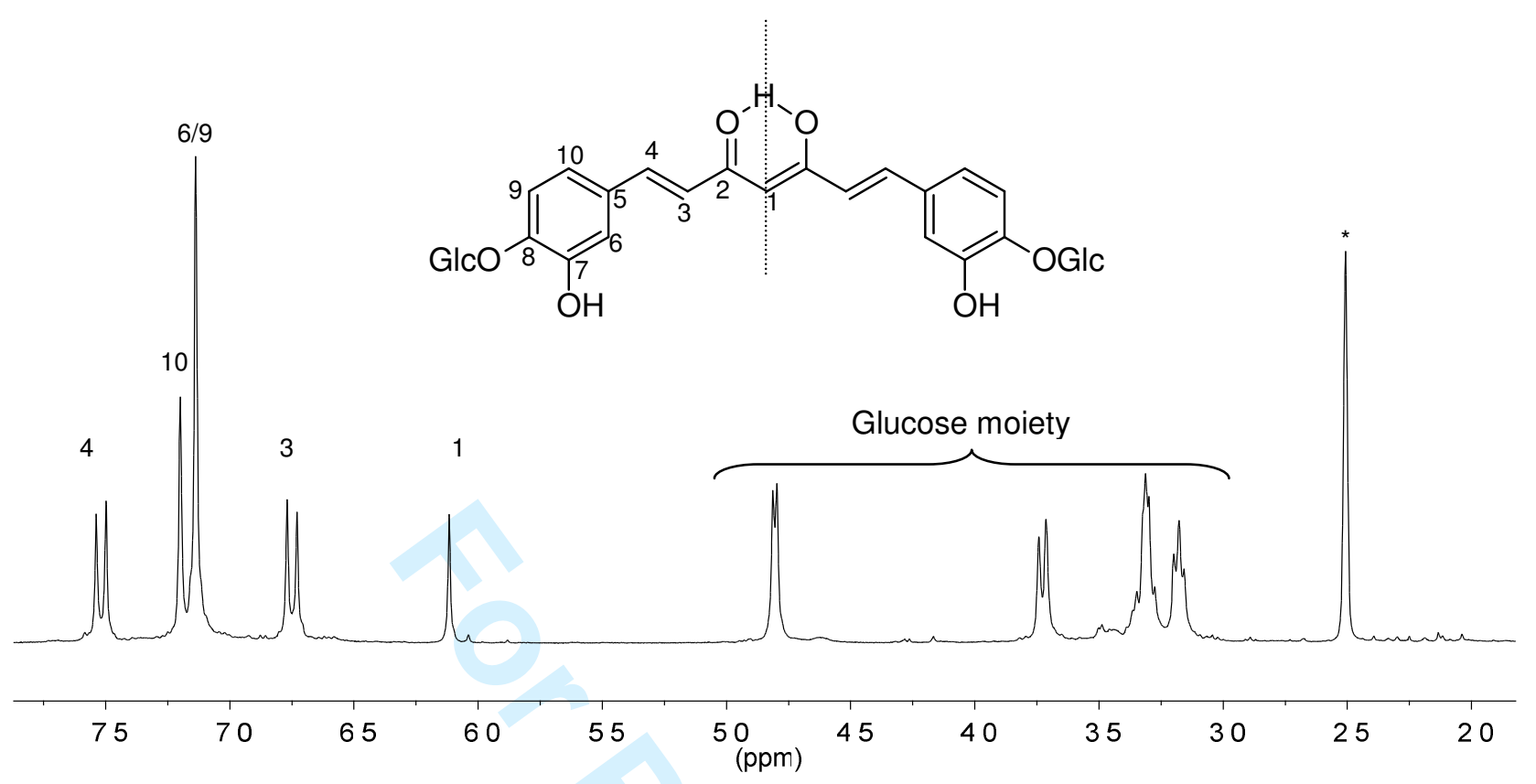

Glucose moiety 
Figure 8
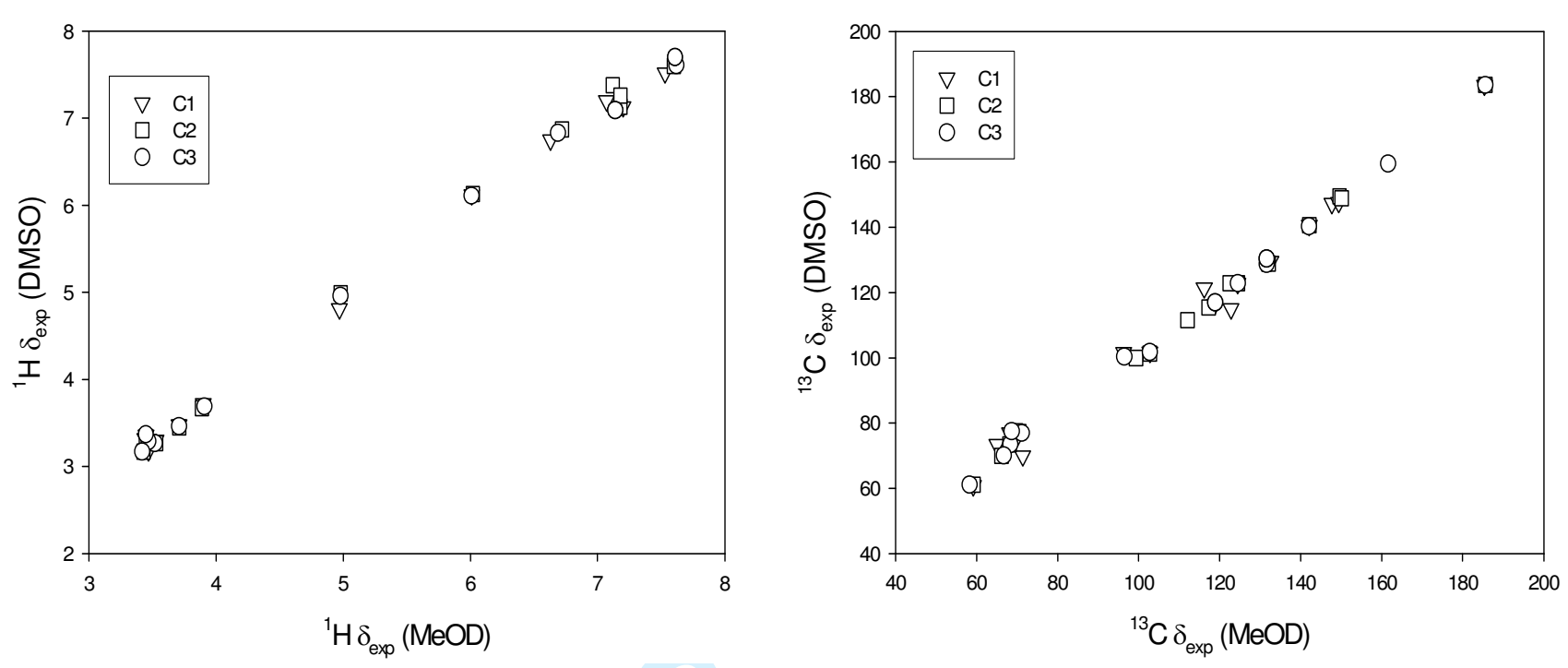

29 


\section{Figure 9}
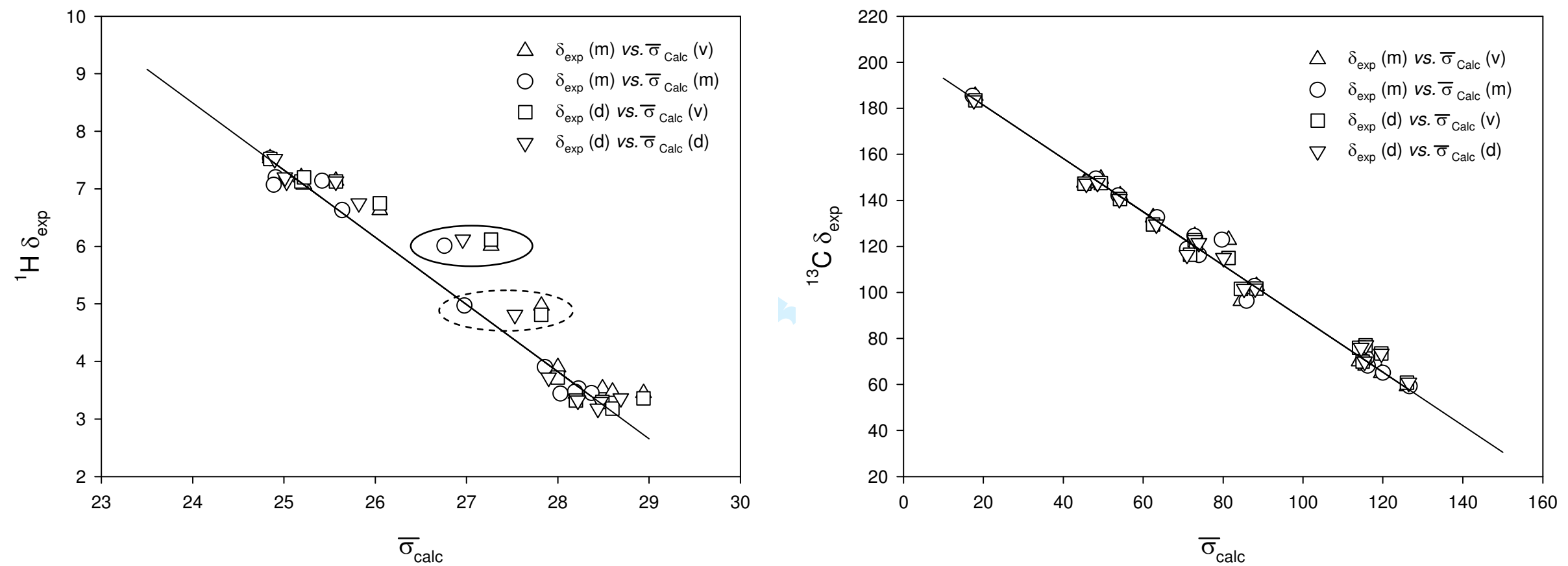
Figure 10

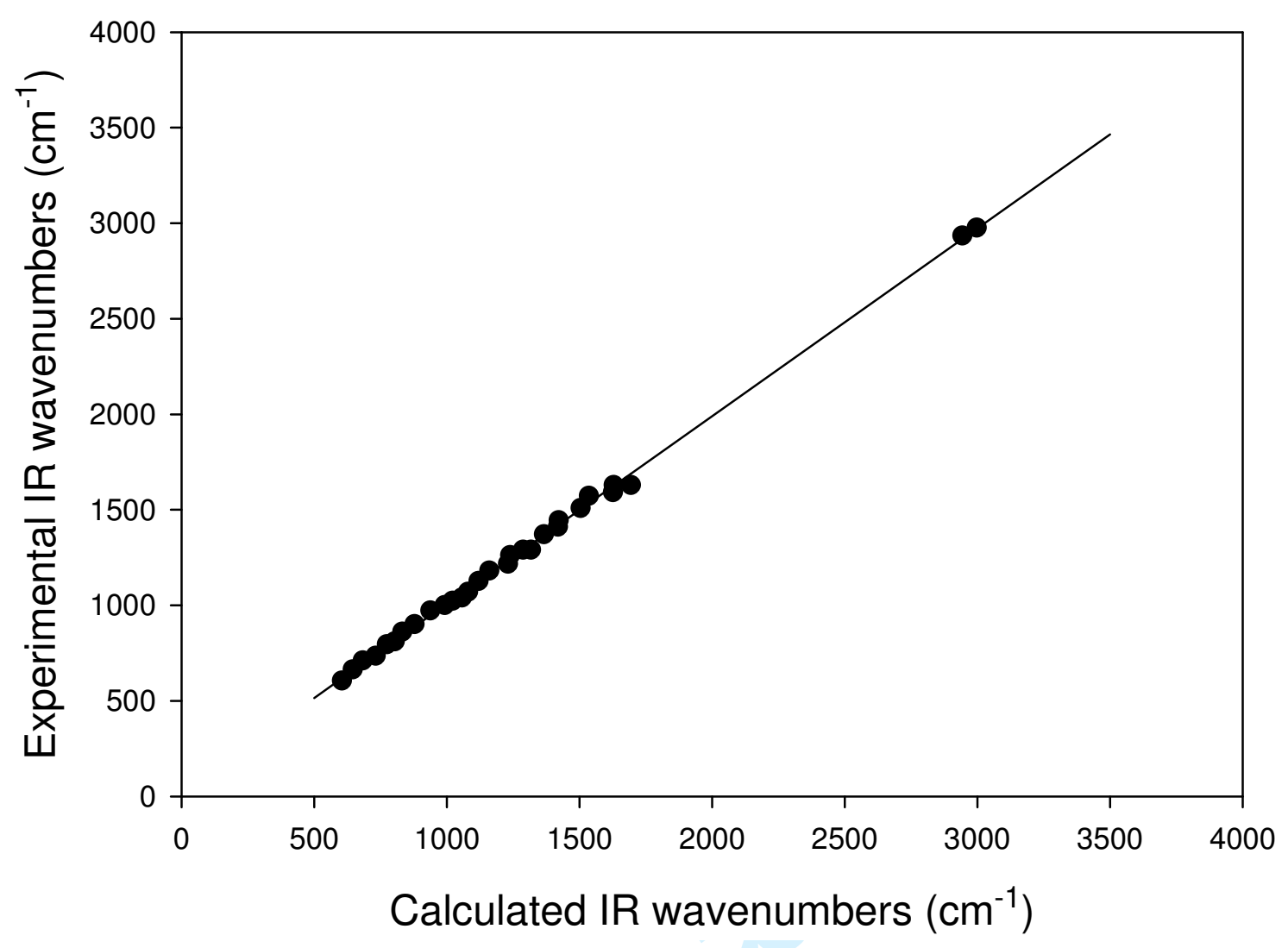

